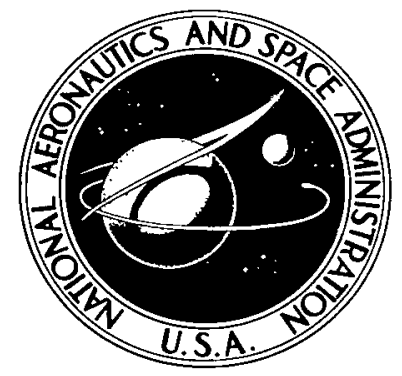

NASA TN D-8211

$\bar{\Xi}$
$\vdots$
$\vdots$
$z$
$\vdots$
$\vdots$

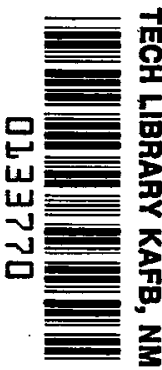

CHARACTERISTICS OF THE NASA LEWIS

BUMPY-TORUS PLASMA GENERATED WITH

HIGH POSITIVE OR NEGATIVE

APPLIED POTENTIALS

LOAN COPY: RETURN TO A.TWL TECHNICAL LIBRARY KIRTLAND AFB, N. M.

J. Reece Roth and Glenn A. Gerdin

Lewis Research Center

Cleveland, Obio 44135

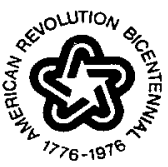

national aeronautics and SPACE administration - WaShINGTON, D. C. • APRIL 1976 
1. Report No.

NASA TN D-8211

2. Government Accession No.

4. Title and Subtitle CHARACTERISTICS OF THE NASA LEWIS BUMPYTORUS PLASMA GENERATED WITH HIGH POSITIVE OR NEGATIVE APPLIED POTENTIALS

7. Author(s)

J. Reece Roth, Lewis Research Center; and Glenn A. Gerdin, NASA-NRC Postdoctoral Resident Research Associate

9. Performing Organization Name and Address

Lewis Research Center

National Aeronautics and Space Administration

Cleveland, Ohio 44135

12. Sponsoring Agency Name and Address

National Aeronautics and Space Administration

Washington, D. C. 20546

15. Supplementary Notes

16. Abstract

The toroidal ring of plasma contained in the NASA Lewis bumpy-torus superconducting magnet facility may be biased to positive or negative potentials approaching 50 kilovolts by applying direct-current voltages of the respective polarity to 12 or fewer of the midplane electrode rings. The electric fields which are responsible for heating the ions by $\mathrm{E} / \mathrm{B}$ drift then point radially outward or inward. The low-frequency $\left(\omega<\omega_{\mathrm{ci}}\right)$ fluctuations below the ion cyclotron frequency appeared to be dominated by rotating spokes propagating in the $\overrightarrow{\mathbf{E}} \times \overrightarrow{\mathbf{B}}$ direction. At frequencies above the ion cyclotron frequency $\left(\omega>\omega_{c i}\right)$, a peak was observed in the emission spectrum that has been tentatively identified as the lower hybrid frequency. Plasma densities up to $10^{1.1}$ particles per cubic centimeter are implied by the observed frequencies. The influence of electric field direction on ion kinetic temperature and ion heating efficiency was investigated. Under equivalent conditions of magnetic field, deuterium background pressure, and midplane electrode voltage, the ion kinetic temperatures and ion heating efficiencies were virtually the same when all 12 midplane electrodes were positive or negative. Deuterium ion kinetic temperatures ranging from 340 to 2500 electron volts were observed in these experiments. The ion heating efficiency ranged from 5 to 22 percent, increased with increasing background pressure or magnetic field, decreased with increasing anode voltage, and was higher in the high-pressure mode than in the low-pressure mode of operation.

17. Key Words (Suggested by Author(s))

Plasma heating

Bumpy torus

Hot ions

Plasma diffusion

Plasma confinement

19. Security Classif. (of this report) Unclassified
21. No. of Pages

63
22. Price"

$\$ 4.25$

* For sale by the National Technical Information Service, Springfield, Virginia 22161 


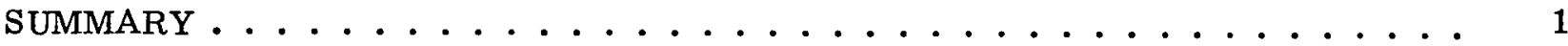

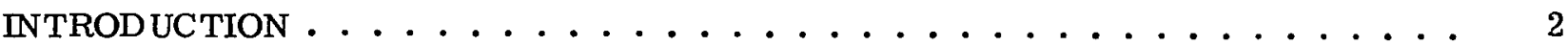

GROSS PLASMA CHARACTERISTICS . . . . . . . . . . . . . . 5

RADIOFREQUENCY EMISSIONS. . . . . . . . . . . . . . . . . . . . 10

Theory of Radiofrequency Emission at Lower Hybrid Frequency . . . . . . . 10

Diagnostic Instruments . . . . . . . . . . . . . . . . . . . . . 12

Moving Peaks in the Radiofrequency Spectrum . . . . . . . . . . . . . 14

Interpretation of Experimental Data . . . . . . . . . . . . . 16

Parametric Variations of Plasma Density . . . . . . . . . . . . . . 17

Surveys of Radiofrequency Spectrum and Spectral Index. . . . . . . . . . 21

LOW -FREQUENCY ELECTROSTATIC POTENTIAL FLUCTUATIONS . . . . . . 23

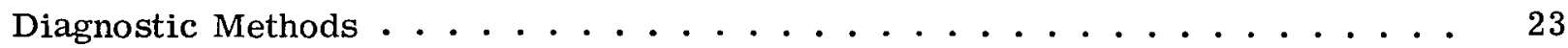

Amplitude of Potential Fluctuations and Spoke Polarization . . . . . . . . 25

Spoke Structure . . . . . . . . . . . . . . . . . . . . . 28

Parametric Behavior of Rotational Mode Number of Spokes. . . . . . . . 31

RELATION OF ION SPOKES TO ION HEATING . . . . . . . . . . . . . 35

FACTORS AFFECTING ION HEATING . . . . . . . . . . . . . . . . 40

PARAMETRIC VARIATIONS OF ION KINETIC TEMPERATURE AND

HEATING EFFICIENCY . . . . . . . . . . . . . . . . . . . . . 47

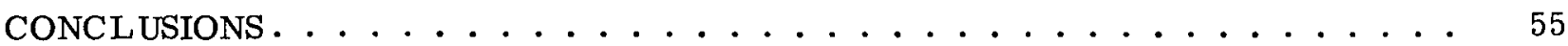

APPENDLX - SYMBOLS . . . . . . . . . . . . . . . . . . 57

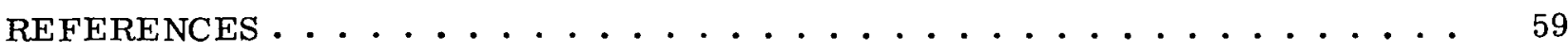




\title{
CHARACTERISTICS OF THE NASA LEWIS BUMPY-TORUS PLASMA GENERATED WITH HIGH POSITIVE OR NEGATIVE APPLIED POTENTIALS
}

\author{
by J. Reece Roth and Glenn A. Gerdin* \\ Lewis Research Center
}

SUMMARY

The toroidal ring of plasma contained in the NASA Lewis bumpy-torus superconducting magnet facility may be biased to positive or negative potentials approaching 50 kilovolts by applying direct-current voltages of the respective polarity to 12 or fewer of the midplane electrode rings. The electric fields which are responsible for heating the ions by $(\vec{E} \times \vec{B}) / B^{2}$ drift then point radially outward or inward. The low-frequency $\left(\omega<\omega_{\text {ic }}\right)$ fluctuations below the ion cyclotron frequency are dominated by rotating spokes propagating in the $(\overrightarrow{\mathrm{E}} \times \overrightarrow{\mathrm{B}}) / \mathrm{B}^{2}$ direction. Above these frequencies the amplitude of the potential fluctuation spectrum obeyed a power-law dependence $\left(\varphi=\varphi_{0} \nu^{-\mathrm{n}}\right)$ with frequency, where the spectral index $n$ was 2.5 for a range of plasma conditions with positive midplane electrodes. At frequencies above the ion cyclotron frequency $\left(\omega>\omega_{\text {ci }}\right)$, a peak was observed in the emission spectrum that has been tentatively identified as the lower hybrid frequency. The implied plasma number densities ranged from $2.5 \times 10^{9}$ to $10^{11}$ particles per cubic centimeter in this series of experiments.

The influence of electric field direction on ion kinetic temperature and ion heating efficiency was investigated. The ion heating efficiency is defined as the ratio of the power input to the ion population divided by the total power input. In this steady-state discharge, it was approximately equal to the ion kinetic temperature in electron volts divided by the anode voltage in volts. Under equivalent conditions of magnetic field, deuterium background pressure, and midplane electrode voltage, the ion kinetic temperatures and ion heating efficiencies were virtually the same when all 12 midplane electrodes were positive or negative. A single positive midplane electrode heated ions as efficiently and to the same kinetic temperature as 12 positive midplane electrodes. Ion kinetic temperatures ranging from 340 to 2500 electron volts in deuterium and from 690 to 3360 electron volts in helium were measured. The ion heating efficiency ranged from 5 to 22 percent, increased with increasing background pressure or magnetic field, decreased with increasing anode voltage, and was higher in the highpressure mode than in the low-pressure mode of operation.

\footnotetext{
*NASA-NRC Postdoctoral Resident Research Associate.
} 


\section{INTRODUCTION}

The objective of the NASA Lewis bumpy-torus experiment is to evaluate the potential of the modified-Penning-discharge ion heating method in a bumpy toroidal magnetic confinement geometry as a means of fusion power production. This approach differs from that at other laboratories engaged in fusion research in at least two major ways:

(1) The plasma and magnetic field are both operated in the steady state.

(2) Strong magnetic and electric fields are applied to the plasma. The electric fields not only heat the ions preferentially by $(\overrightarrow{\mathrm{E}} \times \overrightarrow{\mathrm{B}}) / \mathrm{B}^{2} \mathrm{drift}$, but also may have a beneficial effect on plasma stability and confinement.

(3) The necessary fusion technology is being developed in parallel with the physics, particularly superconducting magnet technology and cryogenic, vacuum, and high-voltage techniques.

The origins and history of the basic bumpy-torus magnetic confinement geometry have been reviewed in reference 1. The combination of the modified Penning discharge with the bumpy-torus magnetic confinement configuration was first proposed in 1967 (ref. 2) and has been under active development at the NASA Lewis Research Center since that time. The bumpy-torus confinement geometry consists of a number of magnetic field coils (12 in the Lewis facility) arranged in a toroidal array in the manner shown schematically in figure 1. At Lewis, the basic configuration shown in figure 1 is supplemented by the modified Penning discharge, which is created in this magnetic field by placing 12 or fewer high-voltage electrode rings at the "bumps" between the magnetic field coils, which are contained in grounded dewars.

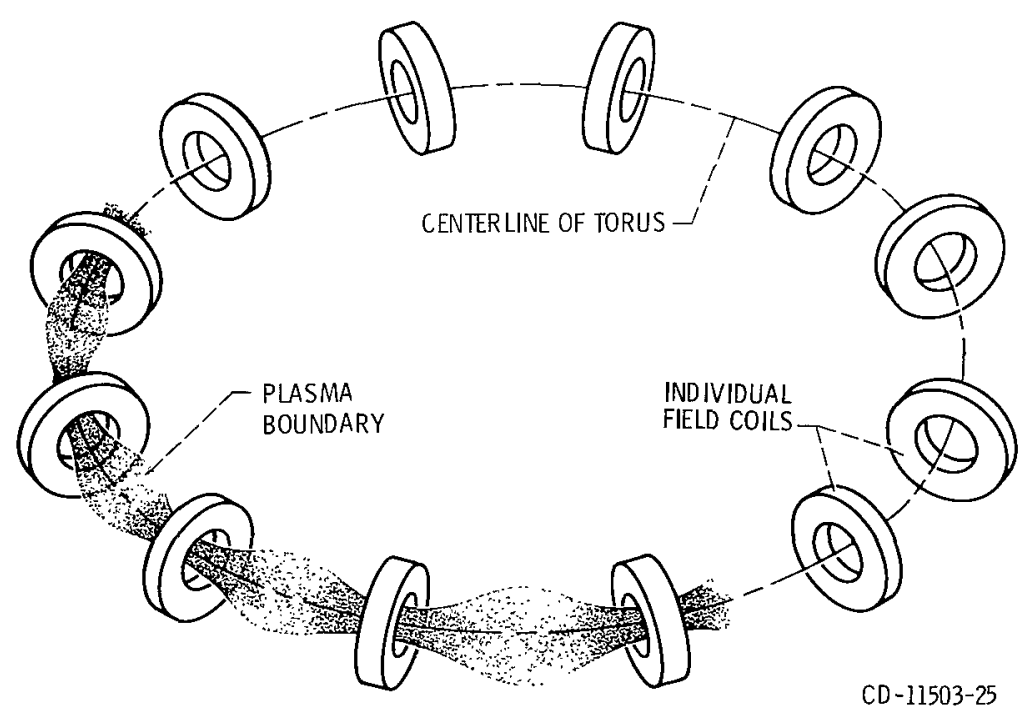

Figure 1. - Schematic drawing of bumpy-torus magnetic confinement geometry. 
An isometric cutaway drawing of the NASA Lewis bumpy-torus facility is shown in figure 2. The 12 superconducting coils, each capable of 3 teslas on its axis, have a 19-centimeter inside diameter, and are arranged in a toroidal array 1. 5 meters in major diameter. The plasma is generated and the ions heated by the midplane electrode rings between the coils. The plasma, as seen through one of the equatorial view ports, is shown in figure 3. The midplane electrode ring, which is biased to high positive potentials in this case, is visible in the for eground. One can also see the sector of plasma at the opposite end of the major diameter to the left of center. The excellent visual and experimental access to the plasma volume are evident. The characteristics and performance of this superconducting magnet facility have been described elsewhere (refs. 3 to 5 ).

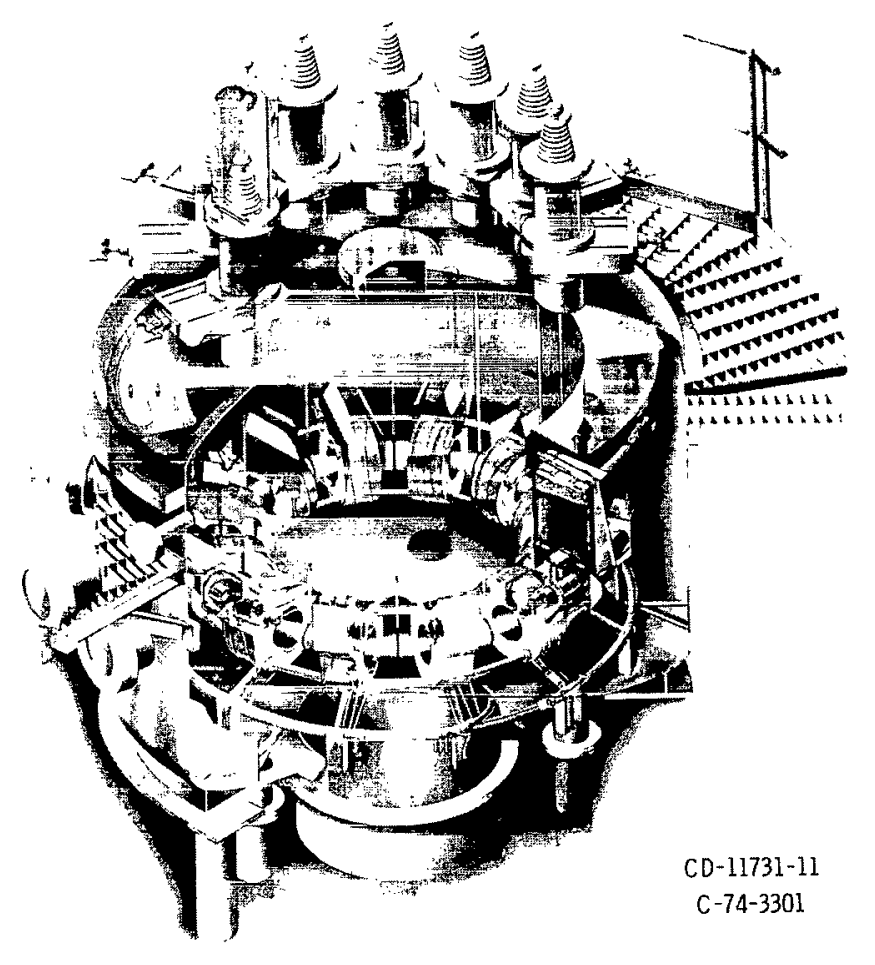

Figure 2. - Isometric cutaway drawing of NASA Lewis bumpy-torus superconducting magnet facility.

The NASA Lewis bumpy-torus project was preceded by an investigation of the modified Penning discharge in a simple magnetic mirror configuration. During these investigations, the presence of kilovolt Maxwellian ions, isotropic in velocity space, was established. The ion heating mechanism was identified and related to $(\overrightarrow{\mathrm{E}} \times \overrightarrow{\mathrm{B}}) / \mathrm{B}^{2} \mathrm{drift}$ of ions in the anode sheath (refs. 6 and 7 ). 


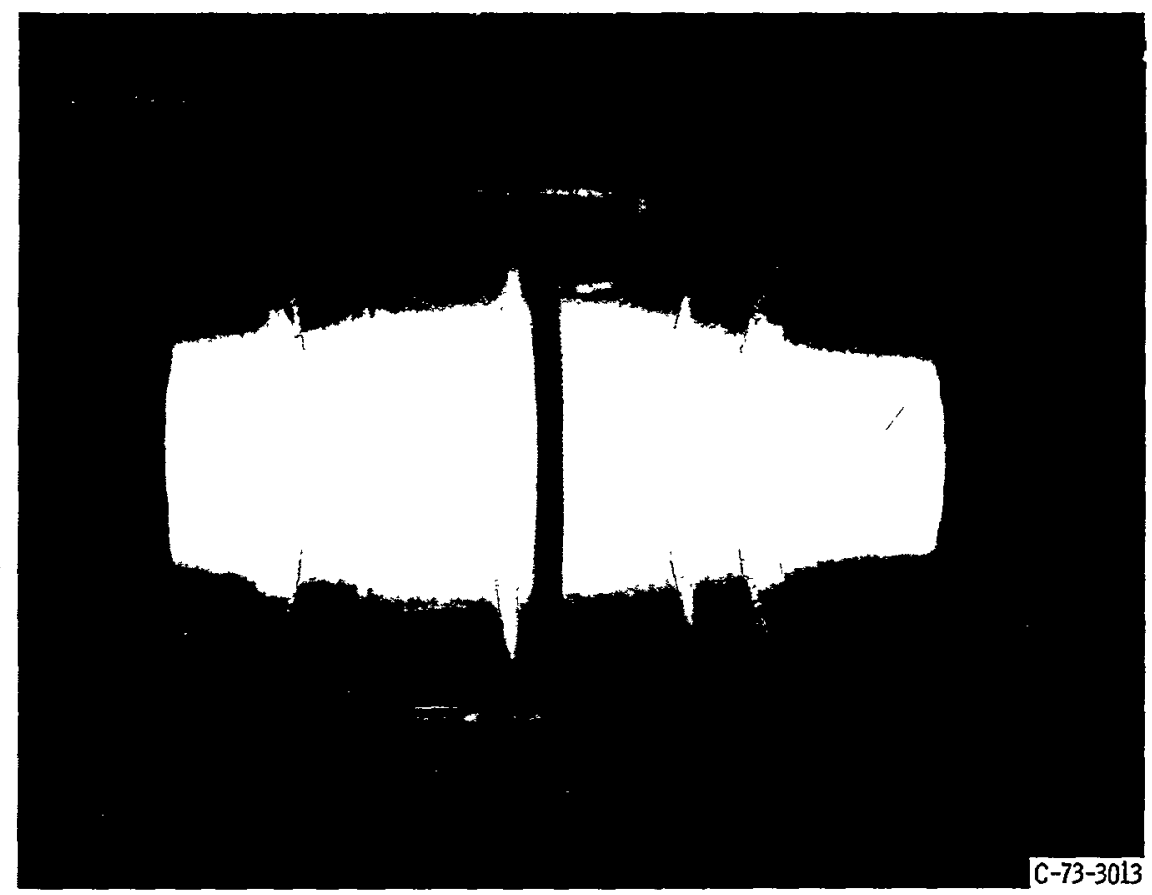

Figure 3. - Bumpy-torus plasma viewed along equatorial plane of torus. (The plasma at opposite diameter of torus is visible to left of the anode ring in the foreground.

Earlier work on the NASA Lewis bumpy-torus plasma was restricted to the application of high positive potentials to the midplane electrodes (refs. 8 to 16). This previous work included spectroscopic determination of the radial profiles of electron temperature and relative number density (refs. 8 to 11 ), studies of the electrostatic potential fluctuations and radiofrequency emission of the plasma (refs. 8, 12, and 13), ion heating and containment in the bumpy-torus plasma (refs. 8 and 14), and studies of impurities and outgassing resulting from plasma-wall interactions in the bumpy torus (ref. 16). In addition, studies with an ion beam probing system have determined the radial electrostatic potential profile in a modified Penning discharge (ref. 15).

The present investigation is concerned with the characteristics of the NASA Lewis bumpy-torus plasma when the plasma is generated with either high positive or high negative applied potentials on the midplane electrode rings. The gross effects of positive and negative midplane electrode potentials on the plasma characteristics are discussed. The high radiofrequency emissions from the plasma were examined and may provide a useful plasma density diagnostic in future experiments of this nature. Examination of the lowfrequency electrostatic potential fluctuations yielded information on the structure, amplitude, and rotational mode behavior of the $(\overrightarrow{\mathrm{E}} \times \overrightarrow{\mathrm{B}}) / \mathrm{B}^{2}$ driven rotating spokes, which are responsible for ion heating in this plasma. The relation between the rotating spokes and ion kinetic temperature was explored, and some of the geometrical and operational 
factors that influence ion heating were examined. The scaling laws of ion kinetic temperature and ion heating efficiency as functions of anode voltage, background neutral gas pressure, and maximum magnetic field were investigated.

\section{GROSS PLASMA CHARACTERISTICS}

The range of plasma parameters covered in the present series of investigations, which took place between January and June 1975, is given in table I. Nearly all runs in this series were made in deuterium gas, with a small number taken in helium for comparative purposes. The anode voltage, anode current, input power, ion kinetic temperature, and plasma number density were limited by arcing or the heating of sheet metal in the vacuum vessel. Modifications of the apparatus should permit the upper limits of these parameters to be significantly increased. The plasma number densities quoted are based on the frequency of moving peaks in the radiofrequency spectrum. These peaks were identified as the lower hybrid frequency, in a manner to be described later. The errors associated with the anode voltage, anode current, input power, magnetic field, and relative measurements of background gas pressure were less than 5 percent; the repeatability of ion kinetic temperature varied by less than 10 percent; and the error in relative measurements of number density was less than 20 percent.

TABLE I. - RANGE OF PLASMA PARAMETERS

\begin{tabular}{|c|c|c|c|c|}
\hline \multirow[t]{2}{*}{ Quantity and units } & \multicolumn{2}{|c|}{ Deuterium } & \multicolumn{2}{|c|}{ Helium } \\
\hline & $\begin{array}{l}\text { Low } \\
\text { value }\end{array}$ & $\begin{array}{l}\text { High } \\
\text { value }\end{array}$ & $\begin{array}{l}\text { Low } \\
\text { value }\end{array}$ & $\begin{array}{l}\text { High } \\
\text { value }\end{array}$ \\
\hline Anode voltage, $\mathrm{V}_{\mathrm{a}}, \mathrm{kV}$ & 1.0 & 45 & 6.0 & 37.5 \\
\hline Anode current, $I_{p}^{a}, A$ & 0.0010 & 4.30 & 0.028 & 1. 125 \\
\hline Input power, $w_{p}, w$ & 1.1 & 76000 & 280 & 22400 \\
\hline $\begin{array}{l}\text { Maximum magnetic field } \\
\text { strength, } \mathrm{B}_{\mathrm{max}}, \mathrm{T}\end{array}$ & 0.24 & 2.4 & 2.4 & 2.4 \\
\hline $\begin{array}{l}\text { Background neutral gas pres- } \\
\text { sure, } \mathrm{p}_{\mathrm{t}} \text {, torr }\end{array}$ & 5. $3 \times 10^{-6}$ & $8.0 \times 10^{-5}$ & 1. $1 \times 10^{-5}$ & 6. $1 \times 10^{-5}$ \\
\hline Ion kinetic temperature, $T_{i}, e V$ & 343 & 2500 & 692 & 3360 \\
\hline $\begin{array}{l}\text { Radiof requency plasma number } \\
\text { density, } \mathrm{n}_{\mathrm{e}}, \mathrm{cm}^{-3}\end{array}$ & 2. $5 \times 10^{9}$ & $1.05 \times 10^{11}$ & $---\cdots$ & $---n--$ \\
\hline
\end{tabular}




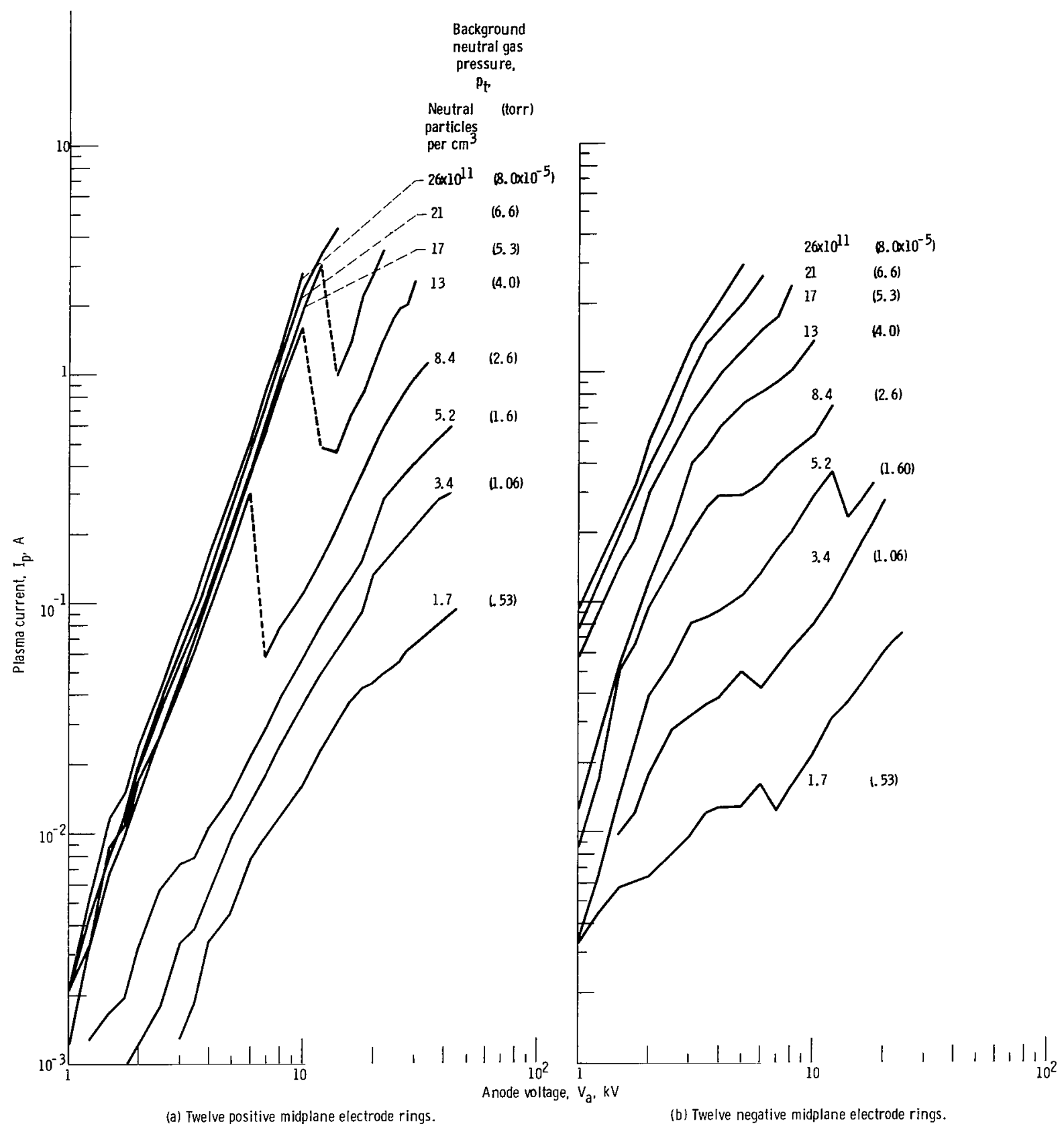

Figure 4. - Current-voltage curves for bumpy-torus plasma at various background pressures of neutral deuterium gas. Maximum magnetic field strength, 2.4 teslas. 
Figures 4(a) and (b) show current-voltage curves for the bumpy-torus plasma at eight different background pressures of neutral deuterium gas and for positive and negative midplane electrode potentials, respectively. With positive electrodes, the currentvoltage curves exhibit two chief modes of operation, the so-called high- and lowpressure modes, which are separated by the sudden transitions indicated as dashed lines on the respective curves. In addition, each mode is characterized by its own slope on the current-voltage diagram. The characteristics of these modes of operation are discussed further in reference 1 . If other conditions are the same, the plasma clearly draws substantially more current with negative midplane electrodes (fig. 4(b)) than with positive; and the negative current-voltage diagram lacks the clear mode structure evident with positive midplane electrodes. The data of figure 4 were taken with 12 midplane electrode rings in the system. A plasma could be generated with as few as one midplane electrode biased to positive potentials, but no plasma could be generated with a single negative midplane electrode ring.

The floating potential of the plasma was measured with the movable Langmuir probe shown schematically in figure 5 . This pneumatically actuated probe could be inserted into the plasma for periods up to 0.7 second and then retracted from the plasma when data were not being taken. This procedure has obvious shortcomings in the context of a hot ion plasma confined in a strong magnetic field, but the results should have some validity because the floating potentials of interest were very much greater than the electron temperature in the plasma.

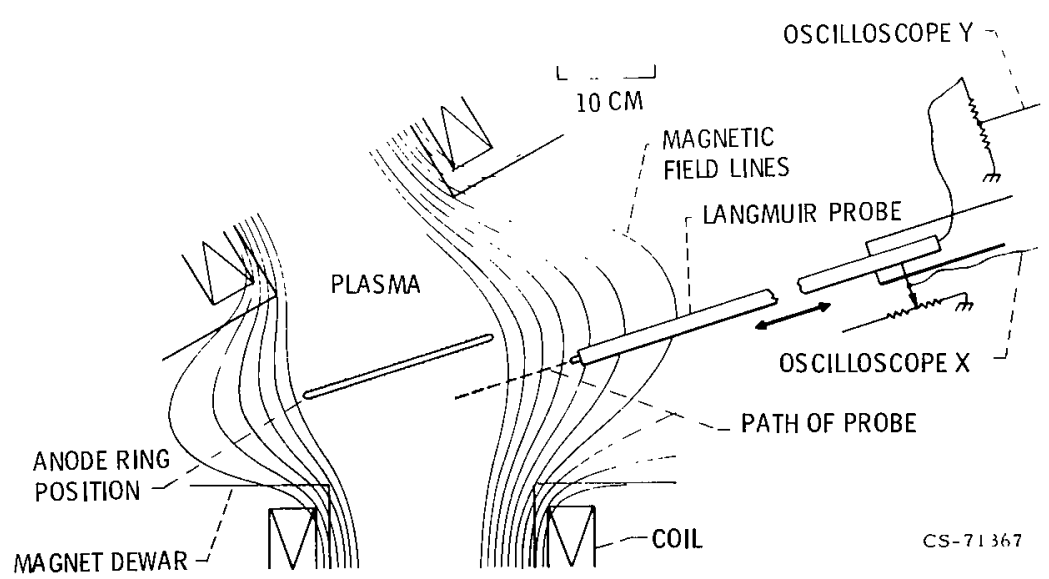

Figure 5. - Schematic drawing of hydraulically actuated, floating Langmuir probe assembly. (Probe capable of dwelling in plasma for 0.7 second.) 


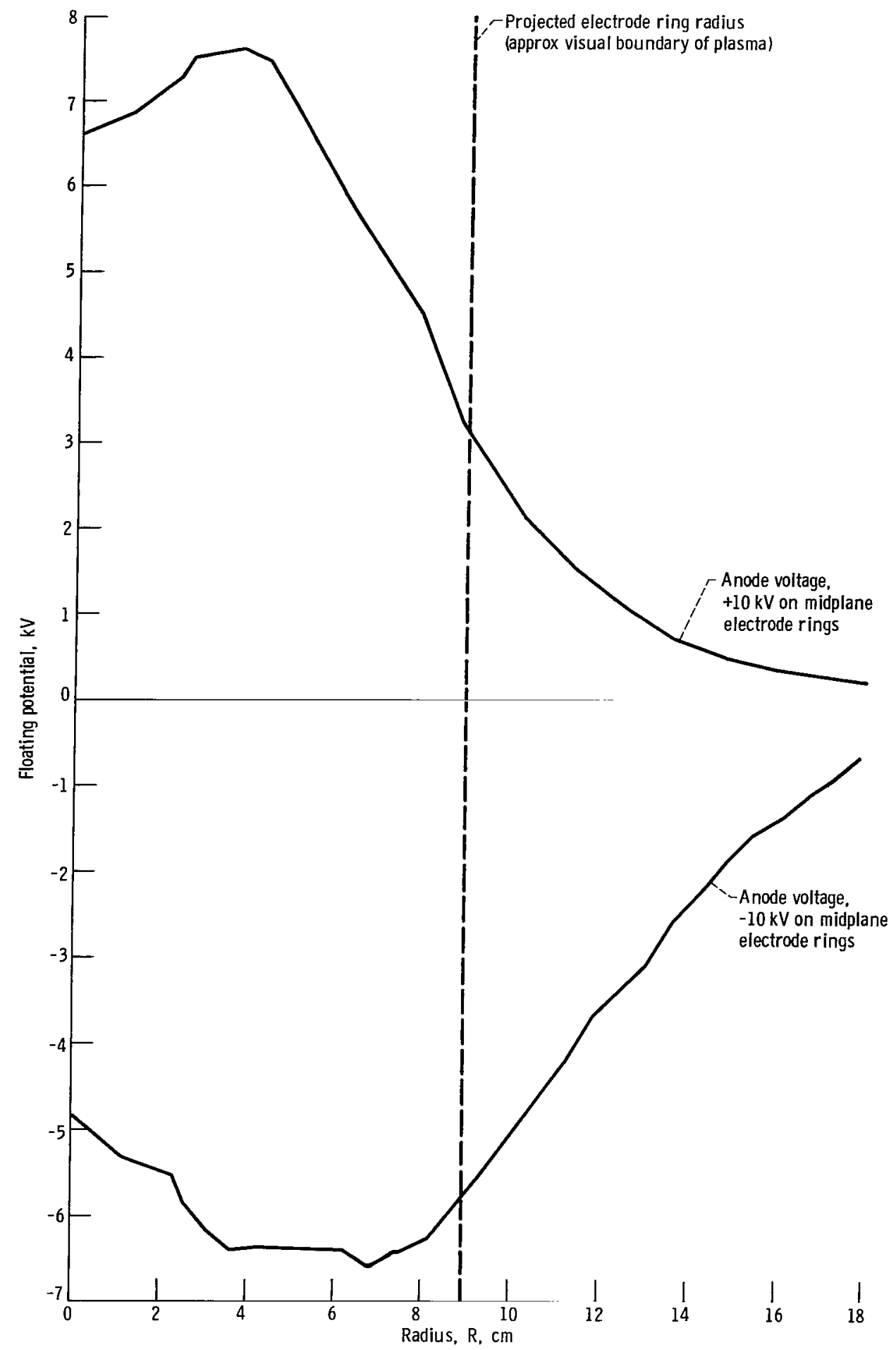

Figure 6. - Radial electrostatic potential profiles ontained with floating Langmuir probe when potentials of tlo kilovolts were applied to midplane electrode rings. No midplane electrode ring located in sector sampled by langmu ir probe. Operating conditions: maximum magnetic field strength, 2.4 teslas; background neutral gas (jeuterium) pressure, $8.4 \times 10^{11}$ neutral particles $/ \mathrm{cm}^{3}\left(2.6 \times 10^{-5}\right.$ torr). 
The results from this diagnostic method are illustrated in figure 6 , which shows the floating potential as a function of radius with a potential of \pm 10 kilovolts applied to the midplane electrodes. The data in figure 6 were taken when the midplane electrode ring was removed from the sector in which the floating Langmuir probe was located, the maximum magnetic field was 2.4 teslas, and the neutral background pressure was $8.4 \times 10^{11}$ neutral particles per cubic centimeter $\left(2.6 \times 10^{-5}\right.$ torr $)$ of deuterium. The radius at which the midplane electrode rings were located in other sectors is indicated by the vertical dashed line. The significant features of these data are that the entire plasma ring floats to high potentials, as high as 75 percent of the applied electrode ring voltage, and that the radial electric fields, which drive the $(\vec{E} \times \vec{B}) / B^{2}$ drifts of the ions and electrons, point radially outward in the vicinity of the plasma boundary for positive midplane electrodes but point radially inward when negative potentials are applied to the midplane electrodes. Data similar to those shown in figure 6 were taken for a variety of pressures and anode voltages with both positive and negative midplane electrode rings. The situation depicted in figure 6 proved to be typical, with the maximum floating potential seldom less than half of the potential applied on the midplane electrode rings. Floating potentials as high as 90 percent of the applied potentials were observed.

These measurements lead to the physical picture of the electric field structure of the bumpy-torus plasma that is indicated schematically in figures 7 (a) and (b) for positive and negative midplane electrode rings, respectively. The entire plasma torus was biased to high positive or negative potentials by application of direct-current voltage to the midplane electrode rings. A relatively small voltage drop existed between the midplane electrode rings and the plasma, but the major potential drop appeared between the plasma and the grounded walls of the coils that surround the plasma volume. These radial electric fields pointed outward from the plasma to the grounded walls when the midplane electrode rings were positive (fig. $7(\mathrm{a})$ ) and radially inward when the midplane electrode rings were negative (fig. $7(b)$ ). As is evident from the data in figure 6, the electric field strength can exceed 1 kilovolt per centimeter.

These electric fields not only are responsible for heating the ions and electrons by $(\vec{E} \times \vec{B}) / B^{2}$ drift, but also may have a significant effect on the stability and containment of the plasma. If no other factors come into play, the outward-pointing electric fields shown in figure 7 (a) should have a detrimental effect on the confinement of plasma ions. The reason is that the ions are at the top of a potential hill and any stochastic process should result in radial transport outward. On the contrary, the situation depicted in figure 7(b) with negative midplane electrodes results in a radial electric field pointing inward, which implies that the ions are in a potential well. Any stochastic process may tend to promote infusion of the ions into the interior of the plasma. 


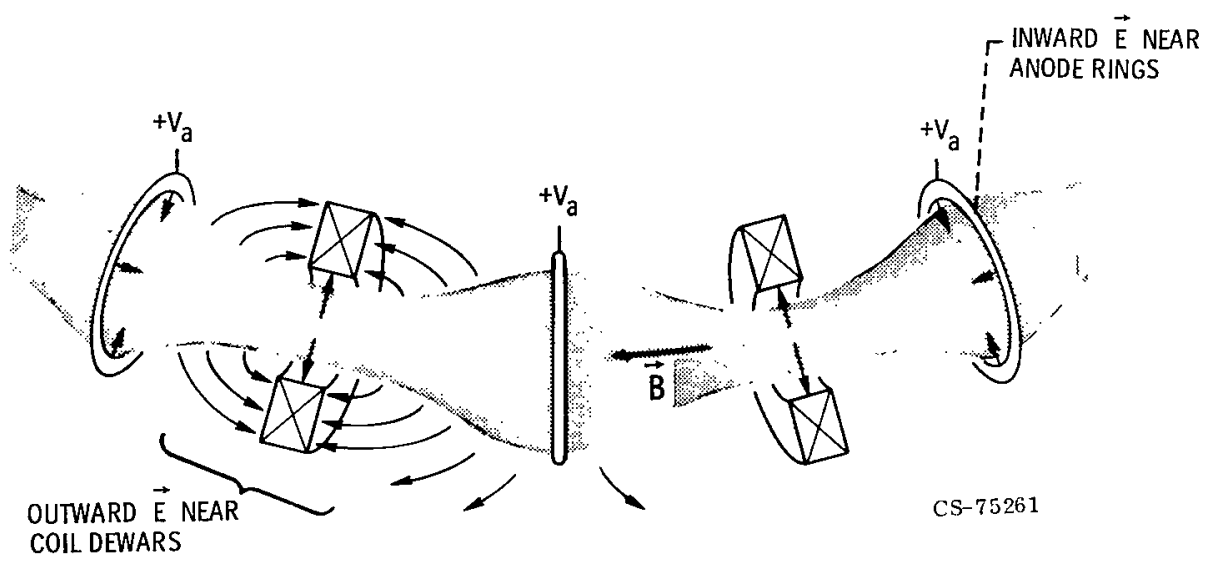

(a) Positive midplane electrode rings.

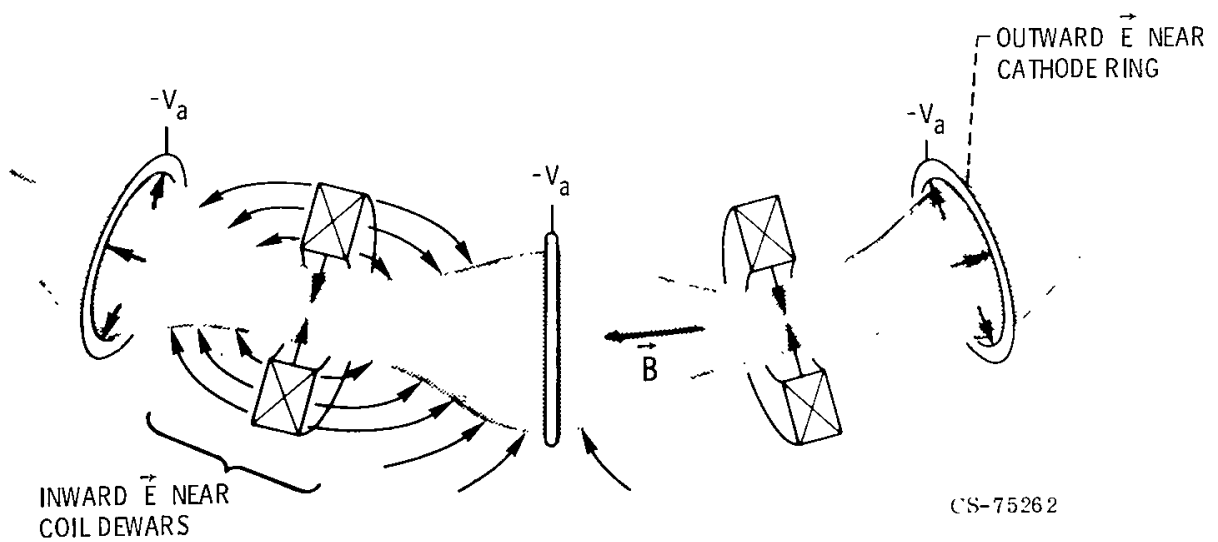

(b) Negative midplane electrode rings.

Figure 7. - Schematic drawing of electric field structure in bumpy-torus plasma. (Note reversal of electric field direction, both in electrode ring sheath and in space between plasma and grounded superconducting magnet dewars. )

\section{RADIOFREQUENCY EMISSIONS}

Theory of Radiofrequency Emission at Lower Hybrid Frequency

If a fluctuation at the lower hybrid frequency could be identified in a plasma, it could be used as a diagnostic tool to measure the density of the ions. The lower hybrid frequency $\omega_{\mathrm{LH}}$ is given by (ref. 17)

$$
\frac{1}{\omega_{\mathrm{LH}}^{2}}=\frac{1}{\omega_{\mathrm{pi}}^{2}+\omega_{\mathrm{ci}}^{2}}+\frac{1}{\omega_{\mathrm{ce}} \omega_{\mathrm{ci}}}
$$


where $\omega_{\text {pi }}$ is the ion plasma frequency, $\omega_{\text {ci }}$ is the ion cyclotron frequency, and $\omega_{\text {ce }}$ is the electron cyclotron frequency. This technique has been successfully used by Burtis (ref. 18) to determine the electron density in the outer magnetosphere on the OGO-3 satellite. The theoretical possibilities for the existence of a plasma fluctuation at the lower hybrid frequency and its observability are first discussed. Then evidence from other experiments for such a fluctuation is presented. And finally investigations of the NASA Lewis bumpy-torus plasma are discussed, including methodology and results.

In their rather extensive treatment of instabilities driven by currents perpendicular to external magnetic fields, Hirose and Alexeff (ref. 19) find that, if the relative drift velocity between the electrons and ions $\vec{V}$ is much greater than the ion diamagnetic drift $\vec{V}_{D}$ and the ion thermal velocity $v_{i t h}$, an instability will exist with a maximum growth rate at the lower hybrid frequency. In this plasma, operating with positive electrode rings, there exists a region of inward-pointing electric fields in the vicinity of the anode dominated by an electron-rich disturbance, called an electron spoke, propagating (ref. 13) in the $\overrightarrow{\mathrm{E}} \times \overrightarrow{\mathrm{B}}$ direction. In the bulk of the plasma, an outward-pointing electric field exists and is dominated by an ion-rich disturbance, also propagating in the $\overrightarrow{\mathrm{E}} \times \overrightarrow{\mathrm{B}}$ direction but opposite to the motion of the electron spoke near the anode ring (fig. 7(a)). The relative direction of the electric field and spoke rotation is reversed when negative potentials are applied to the midplane electrodes (fig. 7(b)). On the boundary between these two regions there should exist a region of high shear where the relative velocity of these two "fluids" is given by $\vec{V}=-\vec{V}_{I S}+\vec{V}_{E S} \sim V_{E S}$, since $\left|\overrightarrow{\mathrm{V}}_{\mathrm{ES}}\right| \gg>\left|\mathrm{V}_{\mathrm{IS}}\right|$ experimentally, and where $\overrightarrow{\mathrm{V}}_{\mathrm{ES}}$ is the $\overrightarrow{\mathrm{E}} \times \overrightarrow{\mathrm{B}}$ drift velocity of the anode sheath and $\vec{V}_{\text {IS }}$ is the $\vec{E} \times \vec{B}$ drift velocity of the bulk plasma.

For the bumpy-torus plasma with an ion temperature of $1 \mathrm{keV}$ in deuterium: $\left|\overrightarrow{\mathrm{V}} / \mathrm{v}_{\mathrm{ith}}\right| \sim 3$ and $\left|\mathrm{V} / \mathrm{v}_{0}\right| \geq 30$. Since Hirose and Alexeff expand their integral dispersion relation in powers of $\left|\mathrm{V} / \mathrm{v}_{\mathrm{ith}}\right|^{-1}$, and since this ratio is $1 / 3$ for the bumpy-torus plasma, there is some concern whether this expansion is valid for our case. On close inspection of the expansion, the terms being thrown away are of the order of 5 percent of those kept, so the expansion appears to be valid. Hence, the work of Hirose and Alexeff would appear to predict that in the shear region between the anode sheath region and the bulk of the plasma, a lower hybrid instability should exist in the bumpy-torus plasma.

Since the probes used to detect these fluctuations are on the outside of the plasma, the radiation must be able to "leak out" of the plasma in some way. Stix (ref. 20) has theoretically investigated the propagation of plasma waves near the hybrid frequencies in the cold, warm, and hot plasma regimes. From his analysis, it appears that an instability at the lower hybrid frequency, once generated, should partially convert to an 
electromagnetic wave propagating to regions of decreasing density and tunneling through the cutoff at $\omega^{2}=\omega_{\text {ci }}^{2}$ (which corresponds to a density of $1.3 \times 10^{7}$ neutral particles $/ \mathrm{cm}^{3}$ at the plasma edge).

Propagation experiments by Moresco (ref. 21) and Hooke and Bernabei (ref. 22) have confirmed Stix's theory experimentally. Thus, it appears that if the instabilities exist at the lower hybrid frequency, they should be observable, since the bumpy-torus plasma has a density profile that decreases with minor radius (ref. 10).

Fluctuations at the lower hybrid frequency have been observed experimentally. Roger Neidigh of Oak Ridge National Laboratory (private communication, March 19, 1970) observed such oscillations in Burnout $V$, which is also an $\vec{E} \times \vec{B}$ device. Ashby and Paton (ref. 23) also observed fluctuations at this frequency when they fired a gunproduced plasma into a solenoidal magnetic field. Here interaction of the streaming electrons with the magnetic fringe fields at the entrance to the solenoid caused a high relative drift between the ions and electrons and hence gave rise to instabilities similar to those subsequently described by Hirose and Alexeff. Thus, it appears that the fluctuations of Hirose and Alexeff were experimentally observed 6 years before their analysis.

\section{Diagnostic Instruments}

The apparatus used to detect high radiofrequency emissions from the plasma is shown schematically in figure 8. The capacitive probe is of a type originally developed by Schmidt (ref. 24) and further studied by Roth and Krawczonek (ref. 25). To minimize cable resonances and the pickup of radiofrequency interference, the capacitive probe

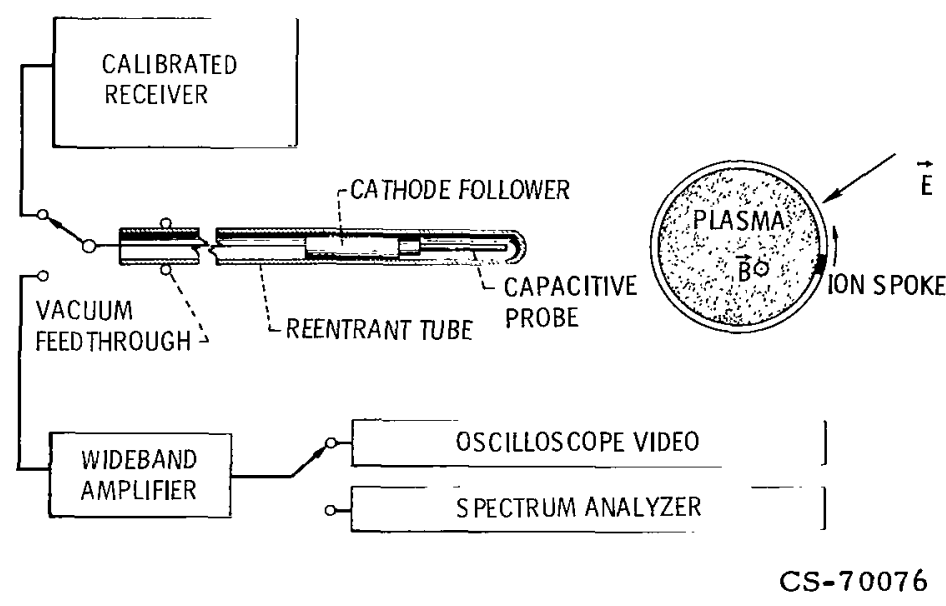

Figure 8. - Schematic of system used to detect radiofrequency emissions from plasma. (Tip of capacitive probe located approximately $5 \mathrm{~cm}$ from plasma; and and cathode follower and probe assembly located inside a Pyrex reentrant tube approximately $1.5 \mathrm{~m}$ long.) 


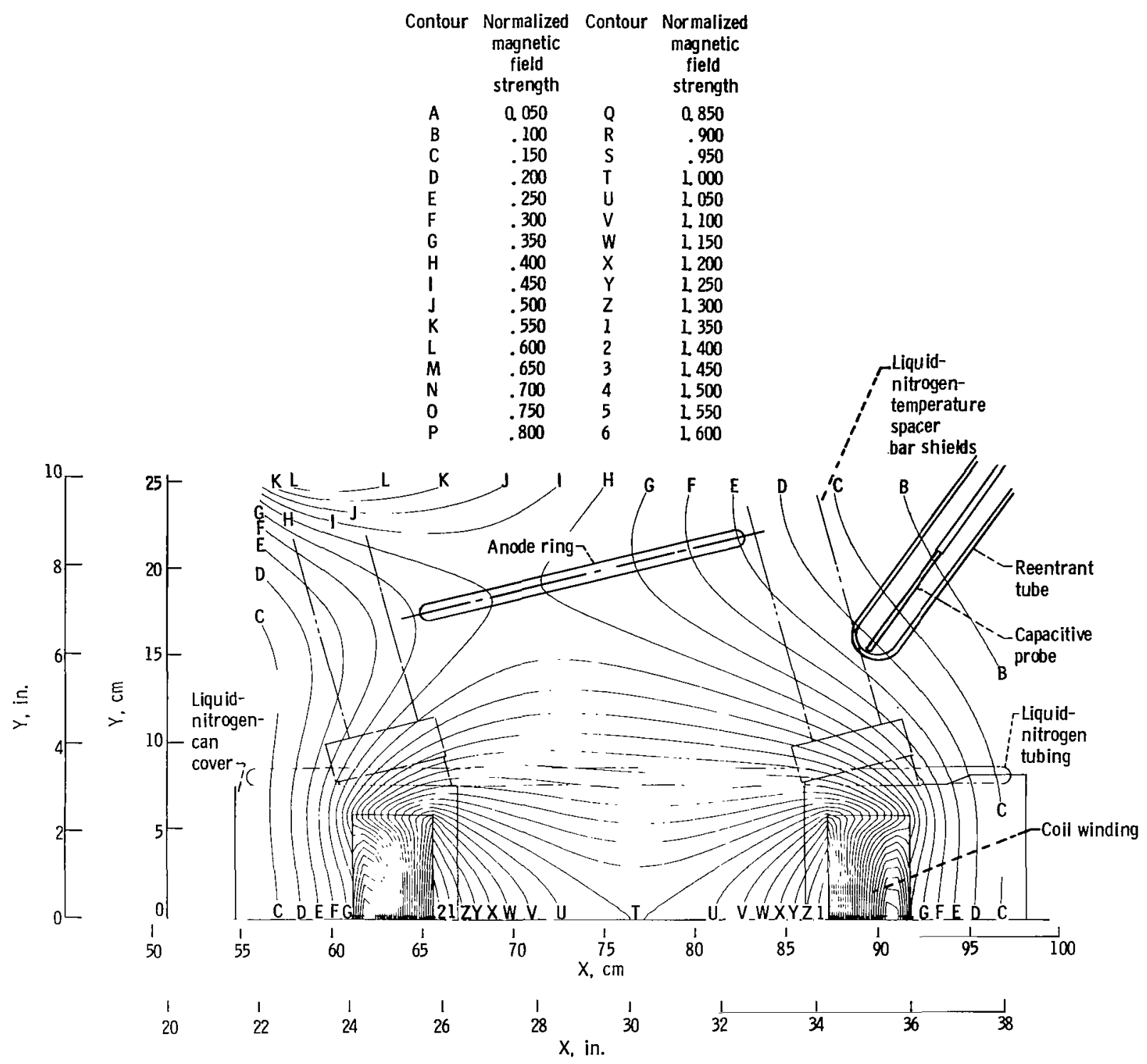

Figure 9. - Location of capacitive probe used for high radiofrequency emission measurements. (Active tip of capacitive probe approximately $4 \mathrm{~mm}$ long; magnetic field strength at capacitive probe tip, 20 percent of $B_{\text {max }}$ )

was directly connected to a high-impedance cathode follower, and the entire assembly was positioned close to the plasma by inserting it in a $1 \frac{1}{2}$-meter-long reentrant Pyrex tube of 2 centimeters inside diameter. The stem of the capacitive probe, the cathode follower, and the cable leading out of the reentrant tube were all triply shielded to minimize external pickup and to reduce cable resonances in the system. The tip of the capacitive probe was located in the equatorial plane of the torus in a position shown in the diagram of figure 9 . The tip of the probe was approximately 5 centimeters from the plasma boundary and was located at a magnetic field strength of about 0.5 tesla when the 
maximum magnetic field $B_{\max }$ was equal to a standard value of 2.4 teslas. The magnetic field at the position of the cathode follower is only a few hundred gauss, and it was determined through comparison tests that this magnetic field had no influence on the frequency response of the capacitive probe - cathode follower combination. The frequency response of this system was almost flat to about 90 megahertz, except for a few cable resonances about 2 or 3 decibels in amplitude.

\section{Moving Peaks in the Radiofrequency Spectrum}

Frequency spectra taken with the radiofrequency probe system previously described are shown in figures 10 (a) and (b) for plasmas generated with positive and negative mid-

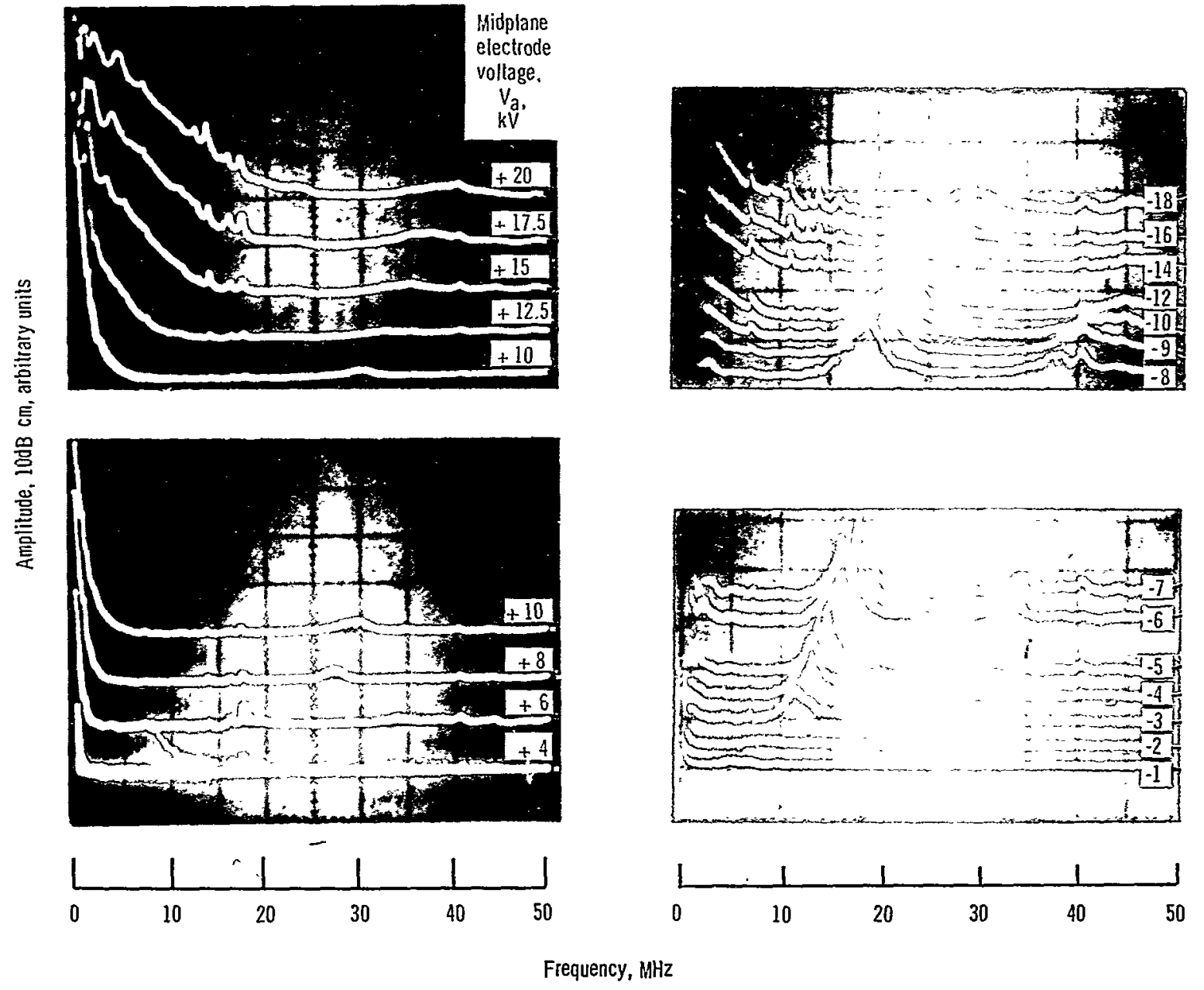

1(a) Example of moving peaks in range 18 to 38 megahertz for background neutral gas (deuterium) pressure of $3.4 \times 10^{11} \mathrm{f}$ neutral particles $/ \mathrm{cm}^{3}\left(1.06 \times 10^{-5}\right.$ torr) and 12 positive midplane electrode rings.

(b) Example of moving peaks in range 8 to 30 megahertz for background neutral gas (deuterium) pressure of $1.7 \times 10^{11}$ neutral particles $/ \mathrm{cm}^{3}\left(0.53 \times 10^{-5}\right.$ torr $)$ and 12 negative midplane electrode rings.

Figure 10. - Raw data showing amplitude of radiofrequency emission in decibels as function of frequency. 


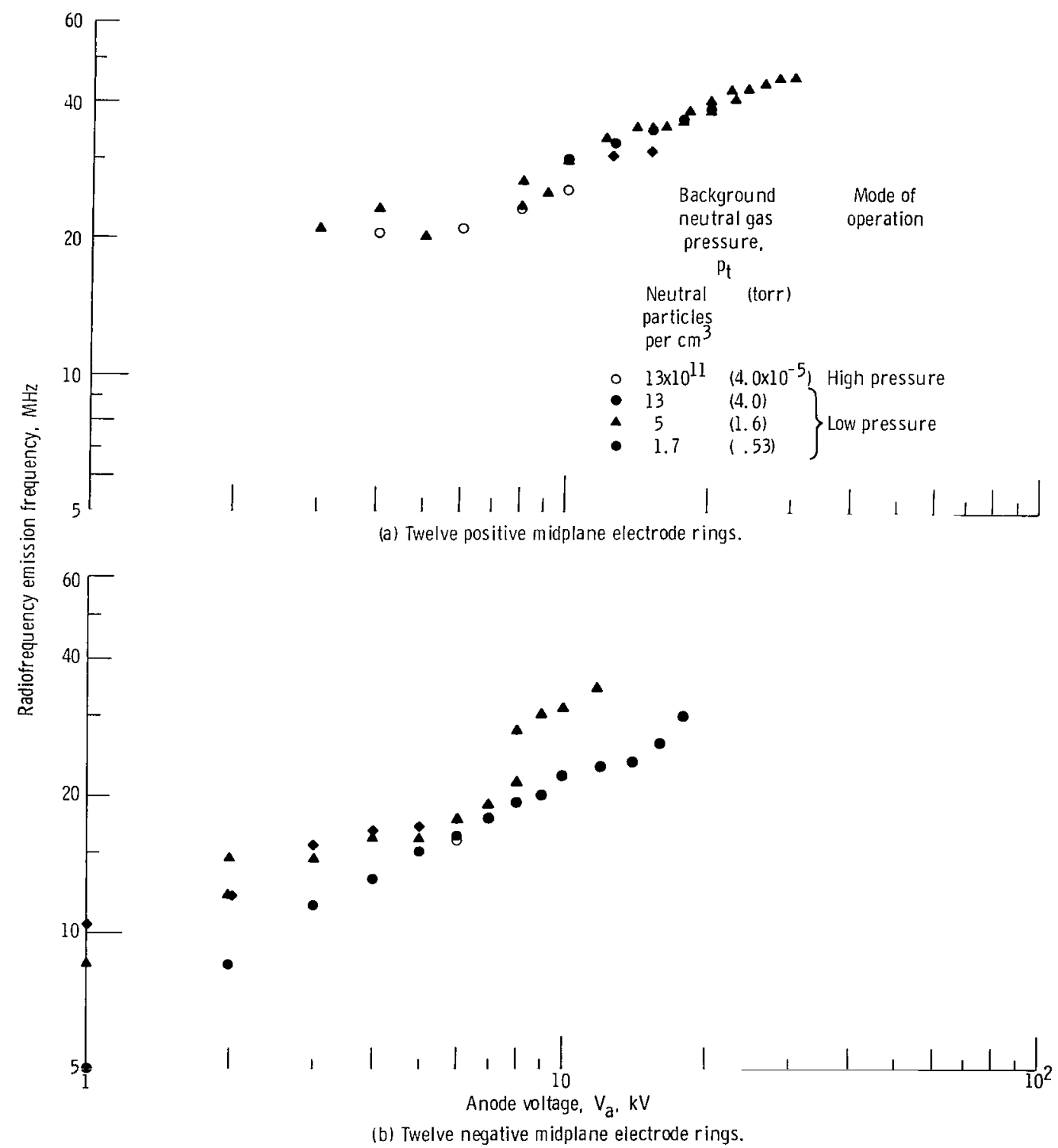

Figure 11. - Frequency of moving peaks as function of anode voltage for various background pressures of neutral deuterium gas. Maximum magnetic field strength, 2.4 teslas. 
plane electrode voltages, respectively. The vertical scale is 10 decibels per centimeter, and the horizontal scale extends from 0 to 50 megahertz. Data are shown for various anode voltages as indicated in figure 10; the successive curves were displaced by 10 decibels in figure 10 (a) and by 5 decibels in figure 10(b). The small stationary peaks between 10 and 20 megahertz and in the vicinity of 40 megahertz are cable resonances that could not be eliminated. In both cases, a moving peak started at low frequencies at the lower electrode voltages and increased in frequency as the electode voltage was increased. The amplitude of this moving peak was only 2 or 3 decibels for positive midplane electrode voltages but was much greater for negative midplane electrode voltages sometimes approaching 20 decibels above the background spectrum (fig. 10(b)).

This moving peak was definitely plasma related. Figures 11(a) and (b) show the variation of the frequency of this moving peak as a function of anode voltage for a maximum magnetic field of 2.4 teslas and for 12 positive and 12 negative midplane electrode rings, respectively. The peak frequency was strongly dependent on the applied anode voltage but was relatively independent of neutral background gas pressure.

\section{Interpretation of Experimental Data}

If the moving peaks exhibited in the radiofrequency spectrum of figure 10 are identified with the lower hybrid frequency at the midplane magnetic field strength, these peak frequencies can be used as a diagnostic tool to identify the plasma electron number density of the emitting region. One problem in obtaining a density from this observed peak is determining from what region of the plasma this radiation is coming. As can be seen from equation (1), the lower hybrid frequency is dependent on both the ion number density and the magnetic field. In the Lewis bumpy-torus plasma, the magnetic field varies spatially by a factor of 2.5 , so this is a serious problem. However, the theory of Hirose and Alexeff predicts the instability to occur in the high-shear region near the midplane electrode ring, where $B=1.0$ tesla for the measurements reported herein. So this field was used for the density calculations from the "moving peak" frequency.

The density was determined by using equation (1) with a magnetic field of 1.0 tesla. An example of how this frequency varies as a function of density for various magnetic fields is shown in figure 12. For densities below $10^{10}$ or above $5 \times 10^{11}$ electrons per cubic centimeter, the density is sensitive to the value of the magnetic field at the point of emission. Fortunately, the densities encountered in this investigation were generally within the band of insensitivity. 


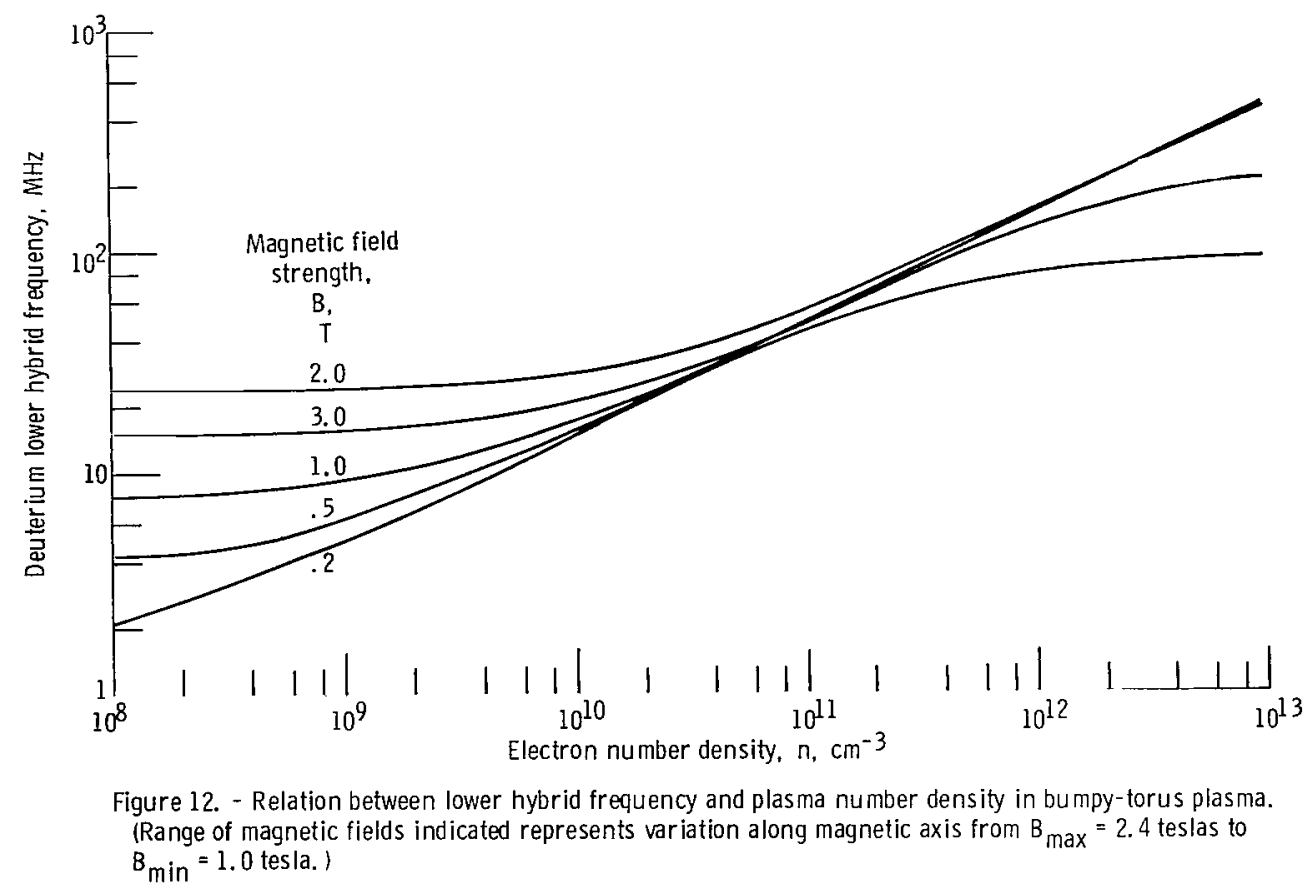

Parametric Variations of Plasma Density

Figures 13(a) and (b) show the electron number densities derived from figure 12 as a function of anode voltage for 12 positive and 12 negative midplane electrode rings, respectively. The densities are relatively independent of background neutral gas pressure and of the modes of operation and vary from about $3 \times 10^{9}$ to $10^{11}$ electrons per cubic centimeter. If other conditions are the same, approximately the same densities result from the use of 12 positive or 12 negative midplane electrode rings.

Figures 14(a) and (b) show the electron number densities derived from moving peak frequencies as a function of anode current for 12 positive and 12 negative midplane electrodes, respectively. These data show that at a fixed anode current, higher plasma number densities may be obtained by reducing the neutral background pressure of deuterium gas. This data trend could result from a limit on the plasma density imposed by charge-exchange neutral production at higher neutral gas pressures.

Figure 15 shows the electron number density derived from the moving peak frequencies for the same background neutral gas pressures, maximum magnetic fields, and anode voltages when the plasma was generated with 12 positive electrode rings (on the ordinate) and 12 negative electrode rings (on the abscissa). The majority of the data fall below the line of agreement, implying that the electron number density may be slightly greater at similar conditions when 12 positive midplane electrode rings are used. However, the scatter is probably too great to justify a firm conclusion. 


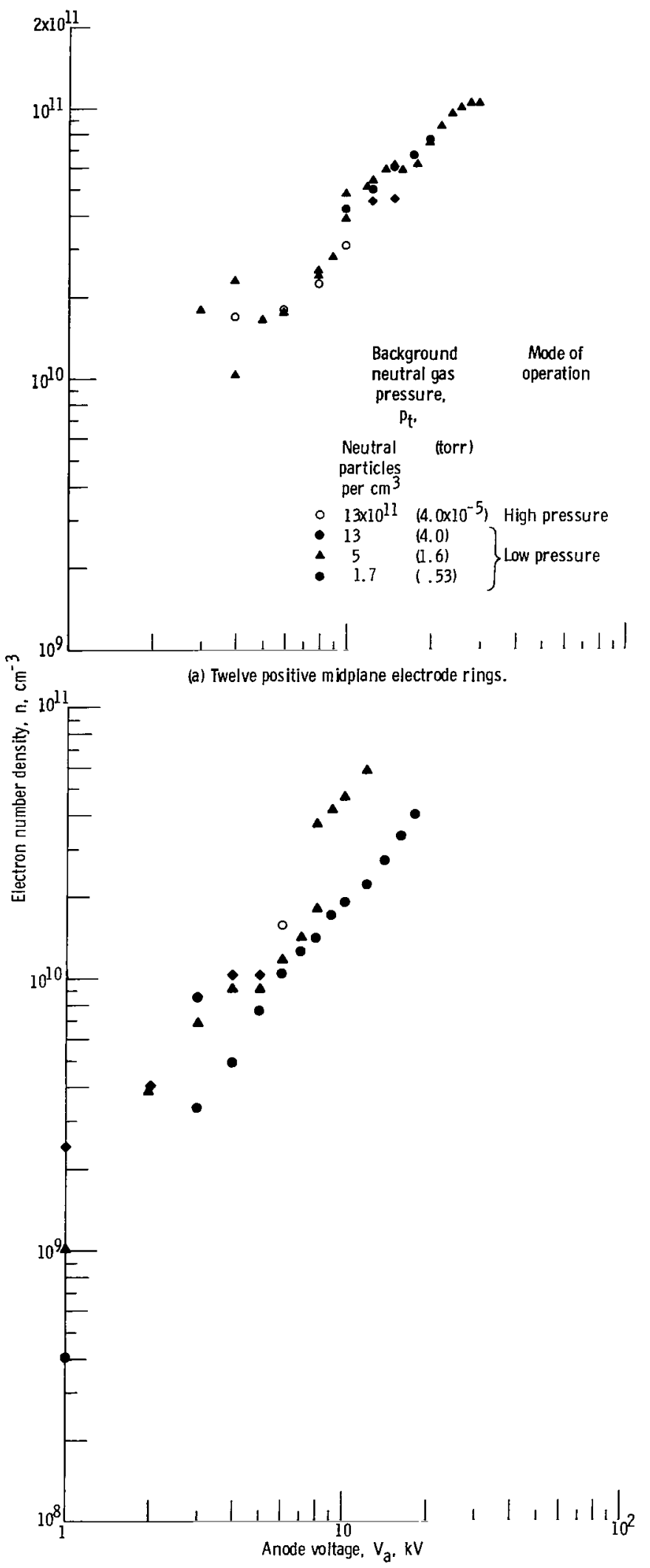

(b) Twelve negative midplane electrode rings.

Figure 13. - Electron number density derived on assumption that moving peak is ion lower hybrid frequency, as function of anode voltage, for various background pressures of neutral deuterium gas. Maximum magnetic field strength, 2.4 teslas. 


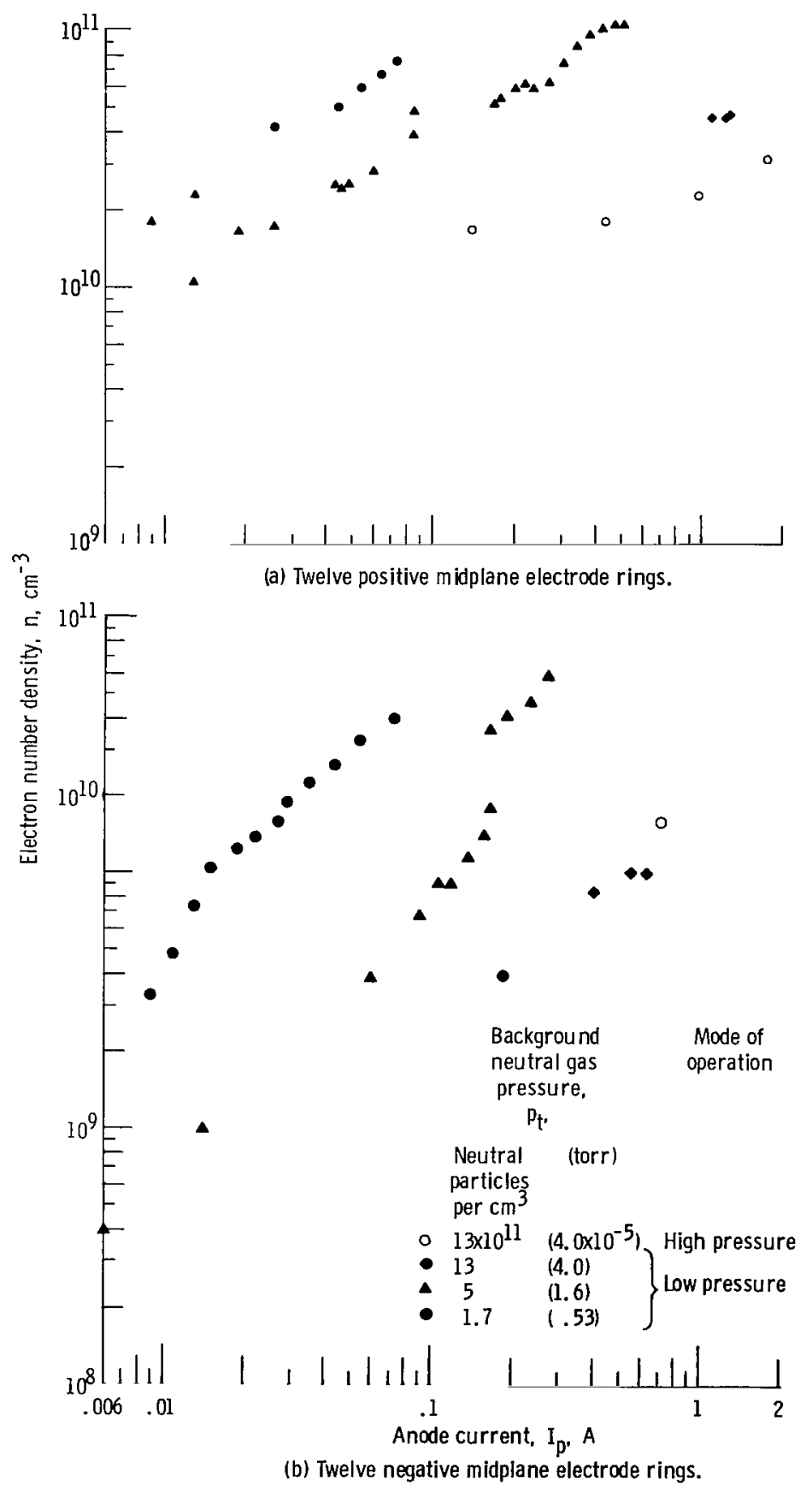

Figure 14. - Electron number density derived on assumption that moving peak is ion lower hybrid frequency, as function of anode current, for various background pressures of neutral deuterium gas. Maximum magnetic field strength, 2.4 teslas. 


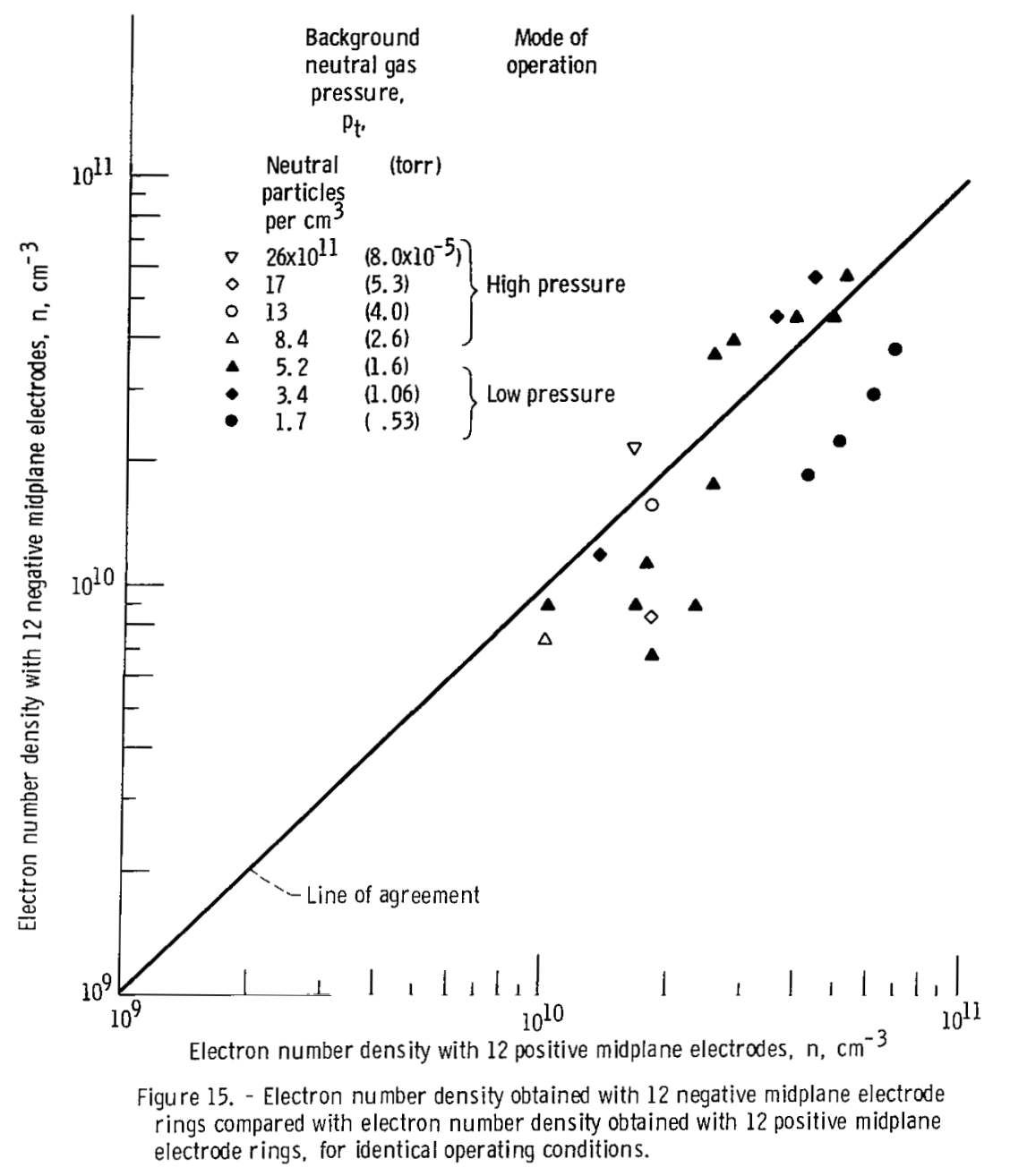

Some corroborative evidence that the number densities derived from the moving peaks are related to the number density of the plasma is suggested in figure 16 . Plotted on the abscissa is the electron number density derived from the radiofrequency emission peak in the manner just described; and plotted on the ordinate is the electron number density, in relative units, obtained from spectroscopic investigations described in references 8 to 10 . The data points plotted in figure 16 were taken at the same conditions of maximum magnetic field strength, neutral background pressure of deuterium, and anode voltage. Comparative data were available only for positive potentials on the midplane electrodes. The data were taken approximately a year apart. A line of $45^{\circ}$ slope was drawn through the data and is a moderately good fit, implying that the electron number density determined from the moving peak is related to the relative electron number density in the plasma as determined by spectroscopic techniques. The fact that the high-pressure-mode data shown in figure 16 have the lowest densities is an artifact of the difficulty of observing the emission peak in the high-pressure mode of 

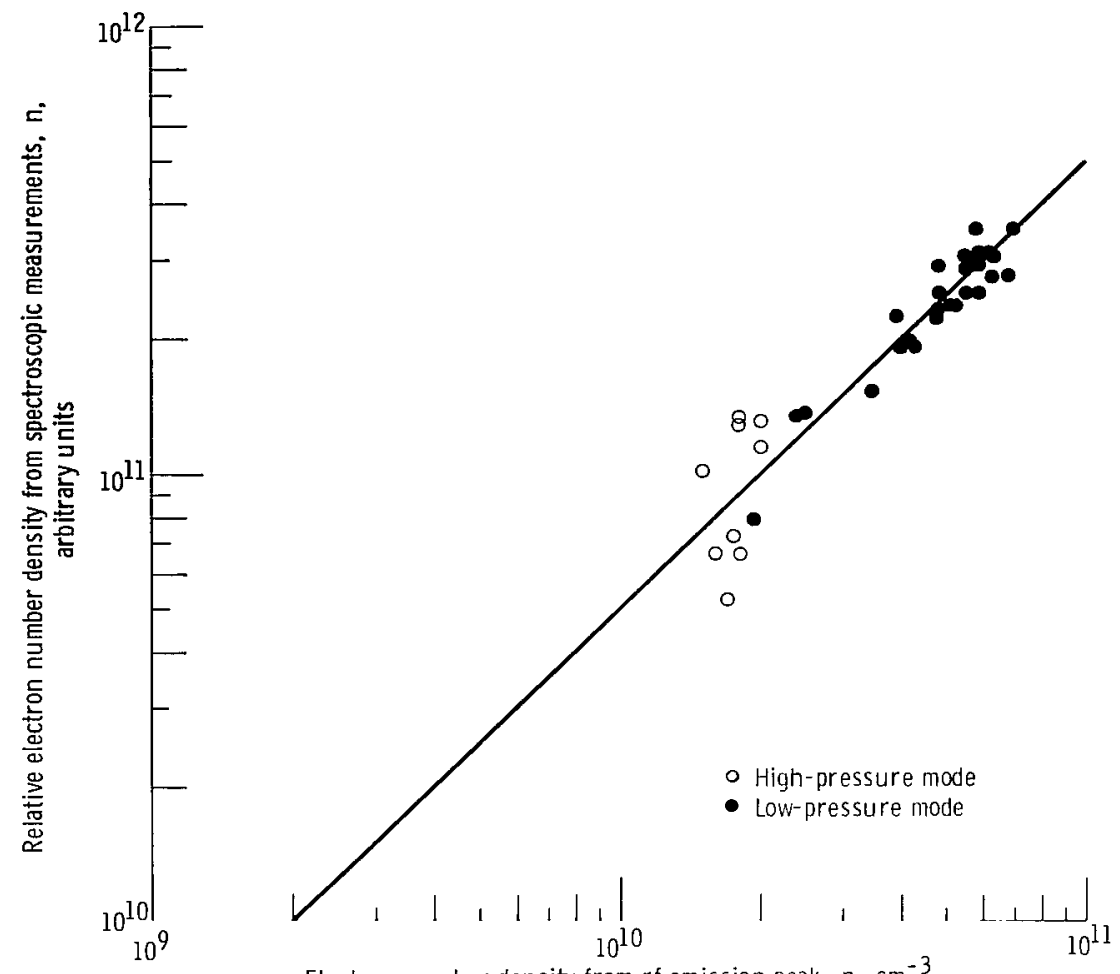

Electron number density from $\mathrm{rf}$ emission peak, $\mathrm{n}, \mathrm{cm}^{-3}$

Figure 16. - Relative electron number density obtained from spectroscopic measurements compared with electron number density obtained on assumption that moving peak is ion lower hybrid frequency, for identical operating conditions.

operation. At higher currents and pressures, where the highest densities in the highpressure mode should occur, the emission peak had faded into the background turbulent spectrum and was not observable.

Surveys of Radiofrequency Spectrum and Spectral Index

Under some conditions of operation, of which the higher voltage curves of figure 10(a) are an example, the amplitude spectrum of electrostatic potential fluctuations was relatively smooth and was found to obey a power-law relation of the form

$$
\varphi=\varphi_{0} \nu^{+\mathrm{n}}
$$

where $\mathrm{n}$ is the spectral index. Several examples of the potential fluctuation spectrum are shown in figures $17(\mathrm{a})$ and (b), which show the amplitude as a function of frequency in the high- and low-pressure modes of operation, respectively. The curves represent the fluctuation spectra observed for the anode voltages indicated. The spectral indices 


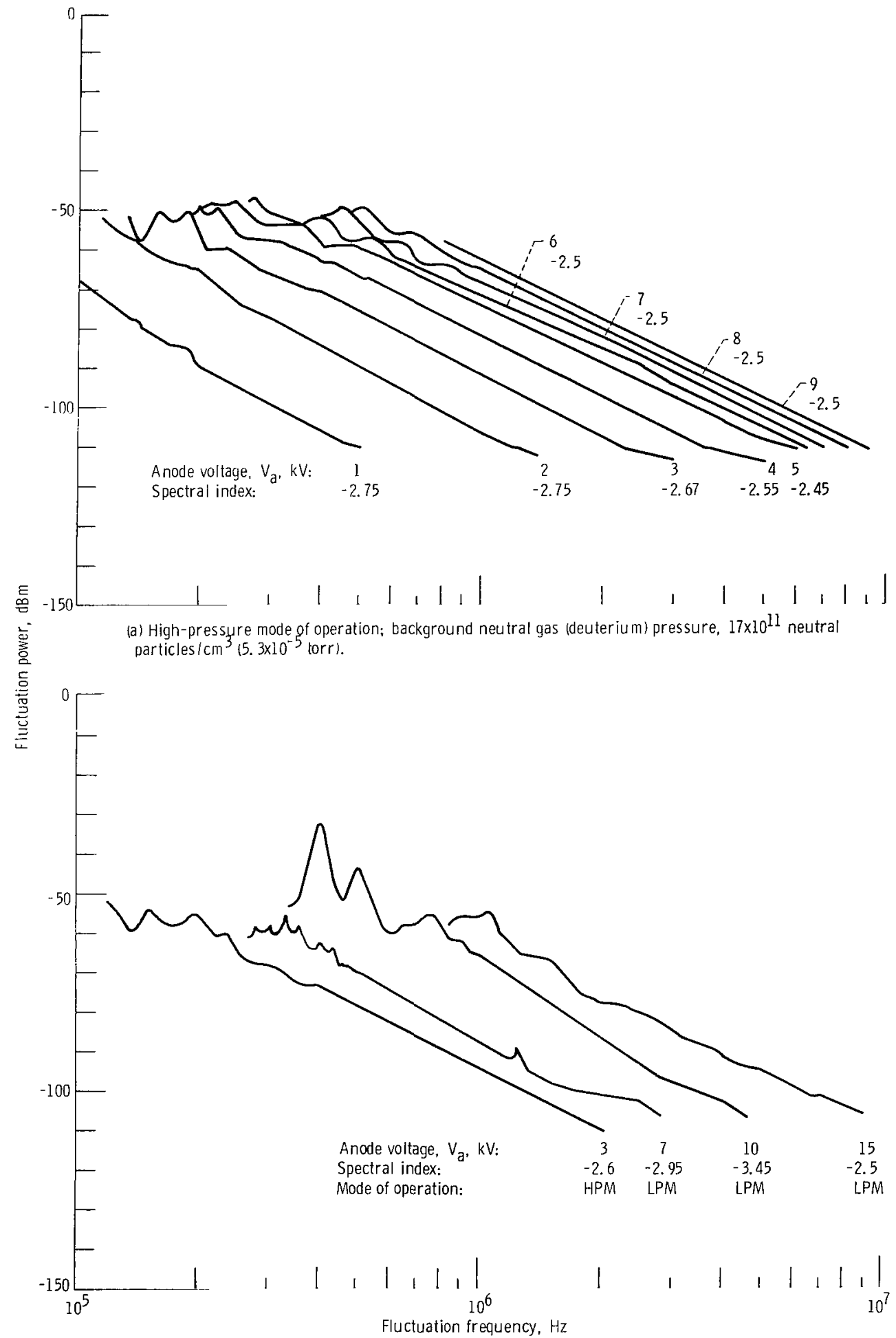

(b) Low-pressure mode of operation (mostly); background neutral gas (deuterium) pressure, $8.4 \times 10^{11}$ neutral particles $/ \mathrm{cm}^{3}\left(2.6 \times 10^{-5}\right.$ torr $)$.

Figure 17. - Spectrum of electrostatic potential fluctuations as function of frequency, in plasma with 12 positive midplane electrode rings and at various positive anode voltages. Maximum magnetic field strength, 2.4 teslas. 
appropriate for the straight-line portions of each curve are also indicated. These spectral indices tend to cluster around the theoretically expected value of -2.5 in the high-pressure mode (ref. 26) but depart substantially from this value in the lowpressure mode of operation. No analogous data were taken with negative midplane electrode voltages. The tendency of the spectral index to agree with a -2.5 power law in the high-pressure mode differs substantially from the behavior observed in an earlier experiment in which a modified Penning discharge was operated in a simple magnetic mirror geometry (ref. 26). In this latter experiment, spectral indices ranging between -1 and -3.5 were observed and depended very much on the plasma operating conditions. An implication of the data shown in figure 17(a) is that in the high-pressure mode of operation the frequency $\omega$ and the wave number $k$ are proportional, a necessary condition for the theory that predicts a $\mathbf{- 2 . 5}$ spectral index. If it is the case that $\omega$ is proportional to $\mathrm{k}$ in the high-pressure mode of operation, this condition may result from the turbulence spectrum being driven by the rotating spokes, the fundamentals and harmonics of which are evident at lower frequencies.

\section{LOW-FREQUENCY ELECTROSTATIC POTENTIAL FLUCTUATIONS}

Surveys of the radiofrequency spectrum such as those illustrated in figures 10 and 17 revealed the peaks and harmonics of coherent oscillations. The amplitudes of these peaks were sometimes several tens of decibels above the background noise spectrum, and their frequencies ranged from a few tens of kilohertz to 1 megahertz. The parametric variation of these peak frequencies with the operating conditions of the plasma was very similar to that of the rotating spokes previously observed in the mirror machine pilot-rig experiment (refs. 6 and 7 ).

\section{Diagnostic Methods}

The bumpy-torus plasma was fitted with an array of capacitive probes to study the structure and configuration of these rotating spokes. Figure 18 shows the array of capacitive probes that were used to detect electrostatic potential disturbances, which were rotating in the minor azimuth, at various stations around the toroidal array. All probes were located at least 5 centimeters from the plasma boundary to avoid damage to the probes. Probes A, B, and $\mathrm{C}$ were spaced at $45^{\circ}$ increments in the minor azimuth and were used to detect spoke rotation in that azimuth. The coherence and phase relations of the rotating spoke about the major circumference of the torus were investigated by comparing the signals from probes B, D, F, and G, all of which were on the equatorial plane of the torus. These signals were also compared with signals from probes $\mathrm{A}$ and $\mathrm{C}$, 


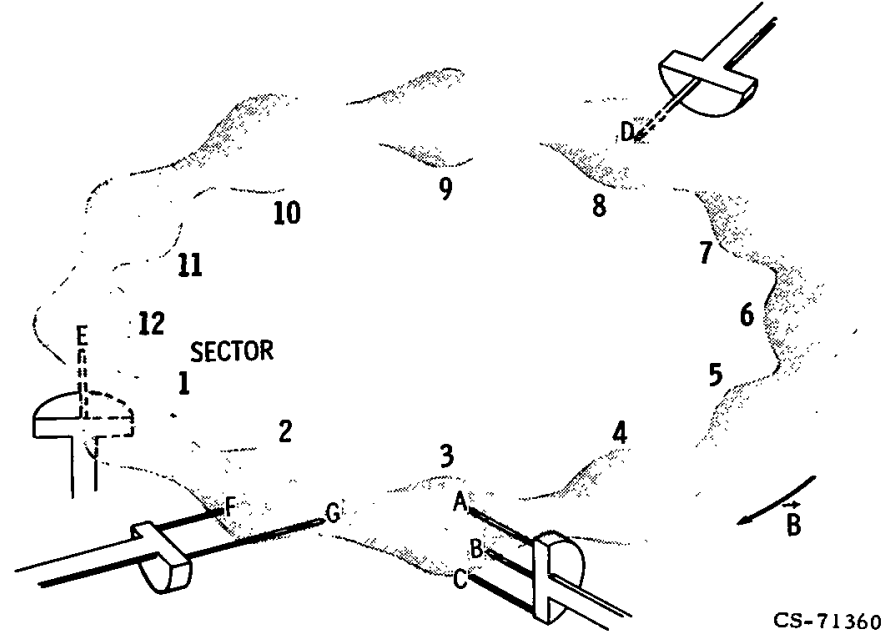

Figure 18. - Location of capacitive probes around bumpy-torus plasma.

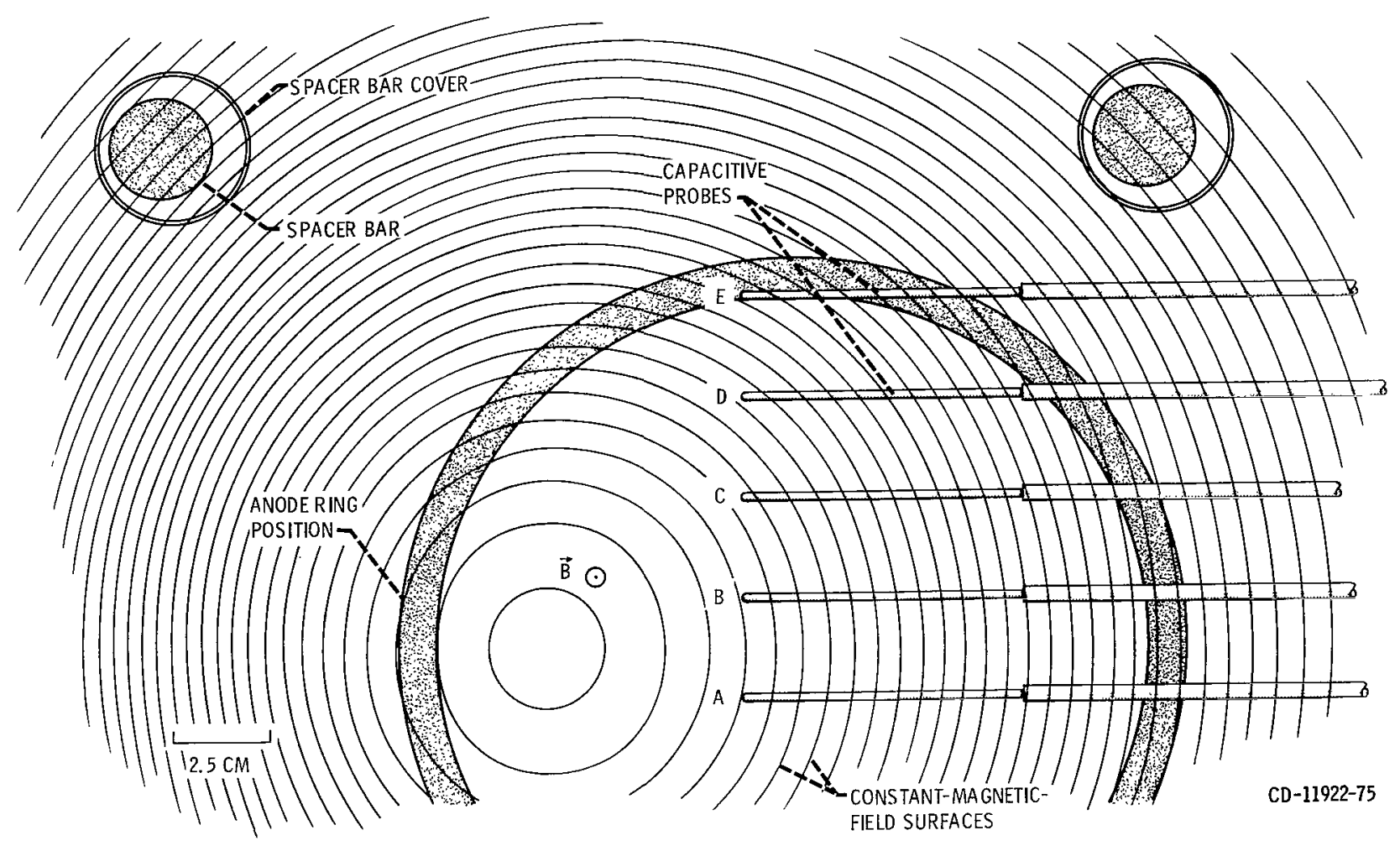

Figure 19. - Position of five capacitive probes for measuring structure of coherent electrostatic potential fluctuations. (Probes A to E located in plane $2.5 \mathrm{~cm}$ from midplane electrode ring in sector 3. ) 
which were at $\pm 45^{\circ}$ from the equatorial plane and with signals from probe $E$, which was $90^{\circ}$ below the equatorial plane. A capacitive probe was additionally located in sector 5 for some of the experimental runs discussed here.

Figure 19 shows an array of five capacitive probes that were used to investigate the radial and azimuthal structure of the rotating spokes. These probes were located 2. 5 centimeters apart on a straight vertical line that was displaced 1.25 centimeters inward from the axis of the anode ring. They were located 2.5 centimeters from the midplane of sector 3 , and the anode ring was in place in that sector. The circular contours are of equal magnetic field strength. Since these five probes were immersed in the plasma, it could not be operated at electrode voltages above 2 kilovolts without risking damage to the probes. As a result, the data on the internal structure of the spokes were limited to the lowest voltages at which the plasma could be operated.

Amplitude of Potential Fluctuations and Spoke Polarization

Unlike the biased Langmuir probe, the capacitive probes respond only to fluctuating electrostatic potentials. The probes were calibrated in such a way that the peak-topeak fluctuation potential existing at the site of the probe tip could be determined for the five probes. These data are shown in figures 20 (a) and (b) for positive and negative midplane electrode rings, respectively. The two sets of data in figure 20(a) show the peak-to-peak amplitude of the electrostatic potential fluctuations at the five probe locations for the high- and low-pressure modes of operation. The electrode voltage was 2 kilovolts, with one set of data taken with an anode voltage of 5 kilovolts. Typically, the highest peak-to-peak fluctuation amplitudes occurred at probe C (fig. 19), which was located approximately 4 centimeters from the plasma axis; and these fluctuations were more intense in the low-pressure mode than in the high-pressure mode. These peak-topeak fluctuation amplitudes ranged from about 10 to 15 percent of the applied electrode voltage. At 5 kilovolts, the peak-to-peak amplitudes ranged up to 3 kilovolts, or 60 percent of the applied potential.

Figure 20(b) shows data taken with 12 negative midplane electrode rings. Both sets of data were taken with only 2 kilovolts applied to the electrode rings. The radial structure of the spokes differs from that of the positive anode ring case shown in figure 20(a). The fluctuation amplitudes increase in magnitude with movement radially outward to the plasma boundary for the high-pressure mode. In the low-pressure mode the maximum peak-to-peak amplitudes appear to occur near probe D (fig. 19), which was located inside the plasma boundary. The peak-to-peak amplitudes of these fluctuations are as much as 10 to 15 percent of the potential applied to the midplane electrodes. The selected data shown in figure 20 are typical, both insofar as their relative magnitude of peak-to-peak fluctuations are concerned and in the radial pattern of intensity of these 


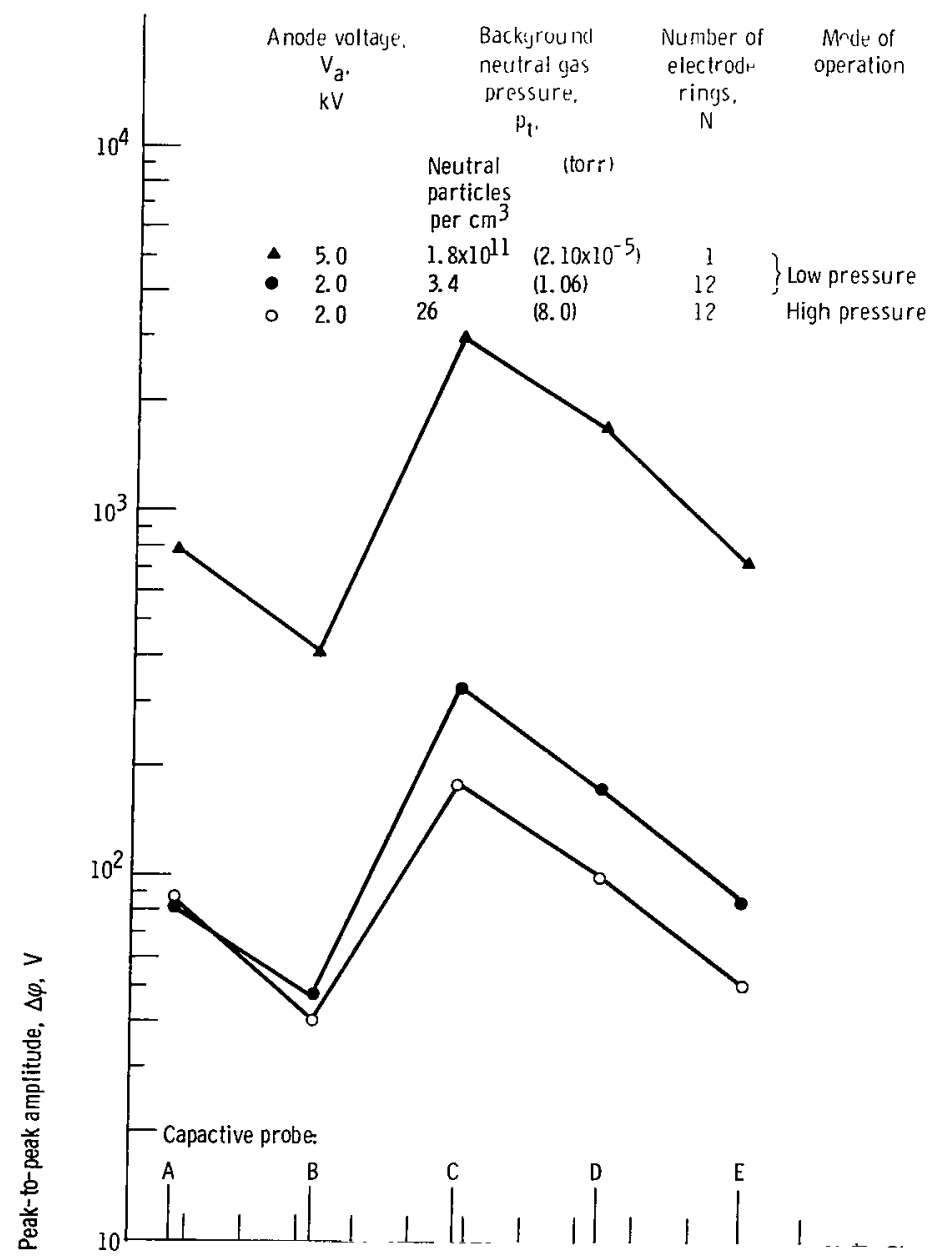

(a) Twelve positive midplane electrode rings.

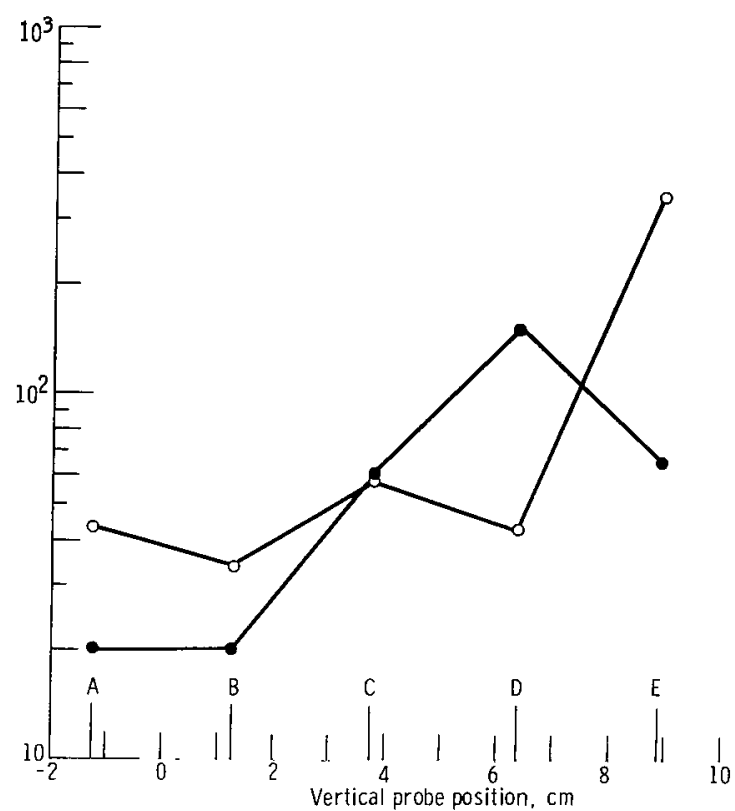

(b) Twelve negative midplane electrode rings.

Figure 20. - Radial profile of peak-to-peak amplitude of electrostatic poten tial fluctuations at capacitive probe tip. Maximum magnetic field strength, 2.4 teslas; high- and low-pressure modes of operation. 


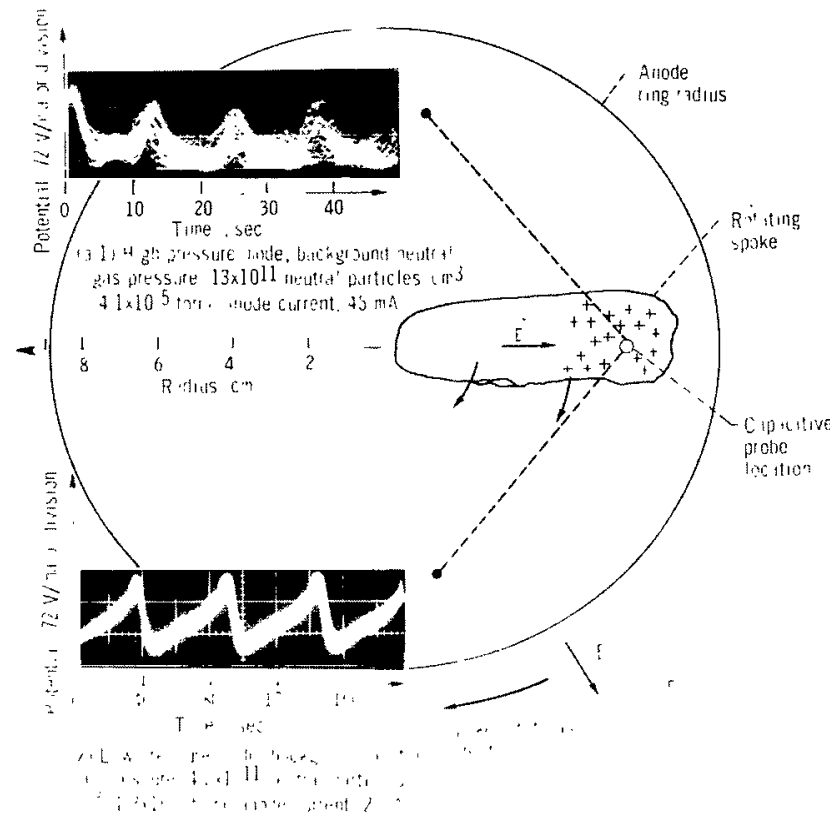

(a) Twelve positive midplane electrode rings.

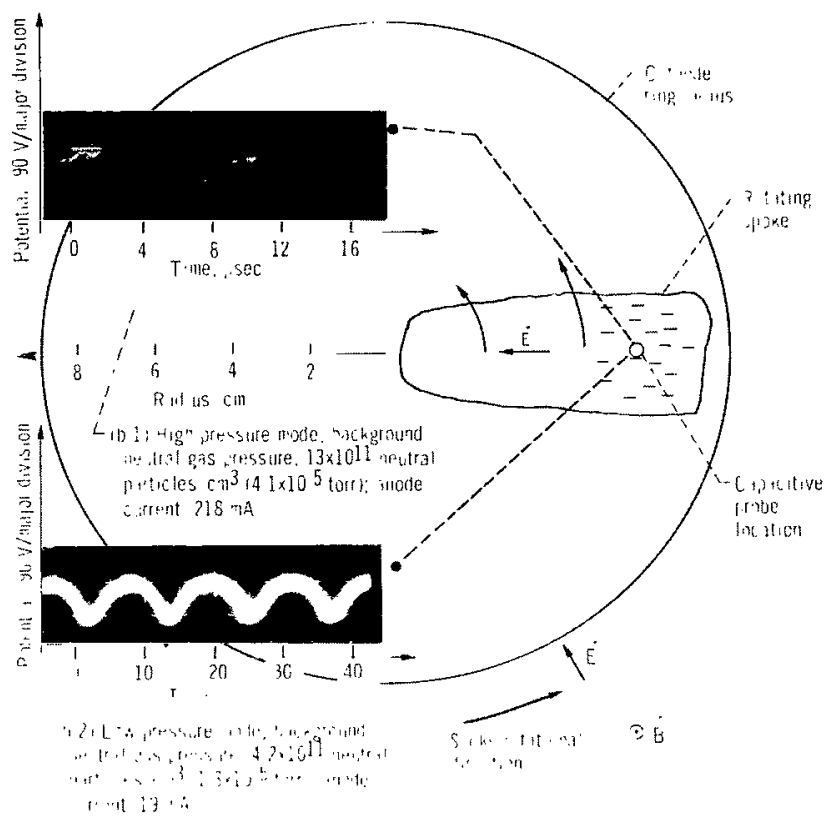

(b) Twelve negative midplane electrode rings.

Figure 21. - Spoke polarızation in high- and low-pressure modes of operation. Maximum magnetic field strength, 2.4 teslas; anode voltage, 2.2 kılovolts.

fluctuations. It is evident from data such as these that the peak-to-peak potential fluctuations in the plasma are far larger than would be expected if strong electric fields were not externally applied. The magnitude of these fluctuations exceeds the electron temperatures measured spectroscopically in this plasma (refs. 8 to 11).

The spokes were found to be polarized by the applied radial electric fields. Data from probe $D$, which was located 6.4 centimeters from the plasma axis, are shown in figures 21 (a) and (b) for the application of 2.2 kilovolts of midplane electrode voltage and for the high- and low-pressure modes of operation, respectively. A positive potential of 2.2 kilovolts applied to all 12 midplane electrodes resulted in the electrostatic potential waveform shown in figure 21(a). These data are typical, both in the magnitude of the peak-to-peak potentials illustrated and in the fact that in the low-pressure mode of operation the signals are of lower frequency and more coherent than the signals obtained from the high-pressure mode of operation. The sign convention on these oscillographs is such that a positive deflection is upward. The waveforms are typically cusped with the cusps pointing upward, implying that the spoke is polarized in such a way that posi- 
tive charges are sweeping by the probe at the position shown. This is the case in both the high- and low-pressure modes of operation.

Figure 21(b) shows the signal from the same probe with a negative potential of 2.2 kilovolts applied to the 12 electrode rings. In this case, the signal from probe $\mathrm{D}$ in both the high - and low-pressure modes of operation is qualitatively different in that the cusps point downward, implying that a bundle of negative charge is sweeping by the probe. The spoke polarization shown in figure 21 is consistent with the results that would be expected from the applied electric field. When positive potentials are applied to the anode ring, as in figure 21(a), the electric field points radially outward, and positive charges are pushed radially outward toward the plasma edge. When the midplane electrodes are negative, as in figure 21(b), the radial electric field points inward. Ions are pushed inward and electrons outward, giving rise to a spoke in which electrons accumulate on the outside of the plasma.

\section{Spoke Structure}

The waveforms obtained from the array of five capacitive probes shown in figure 19 were compared with information from probes at other sectors around the torus to determine the azimuthal mode number and structure of the spokes. The phase lines of the electrostatic potential fluctuations were reduced and are plotted in figures 22(a) and (b) for positive and negative midplane electrode rings, respectively, in the high-pressure mode of operation. Figures 23(a) and (b) show the phase lines of the spoke potential for the low-pressure mode with 12 positive and 12 negative midplane electrode rings, respectively. The solid lines on these graphs give the maximum positive electrostatic potential shown on the oscillographs, and the dashed lines denote the position of one-half of the maximum positive potential on the oscillographs. In figure 22(a) the rotating spokes have an $\mathrm{m}=-2$ configuration; that is, there were two spokes rotating in the sense of electric fields pointing outward, as is consistent with the electric field direction determined from the floating-Langmuir-probe potential measurements shown in figure 6. With positive midplane electrode rings, the outer portions of the spoke lagged slightly behind the inner portions. Figure 22(b) shows the mode structure for 12 negative midplane electrode rings in the high-pressure mode. In this case, the minor azimuthal structure was an $\mathrm{m}=+3$ mode; that is, there were three spokes rotating in the direction appropriate to an inward-pointing radial electric field. In this case, also, the outer portions of the spoke lagged the inner portions. The mode structure for the low-pressure mode of operation is shown in figure 23. The spoke structure is much simpler in this case, an $m=-1$ mode for the positive midplane electrode rings and an $\mathrm{m}=+1$ mode for the negative midplane electrode rings. In both cases the outer portions of the spoke appeared to lead the inner portions by a slight amount, contrary to the 


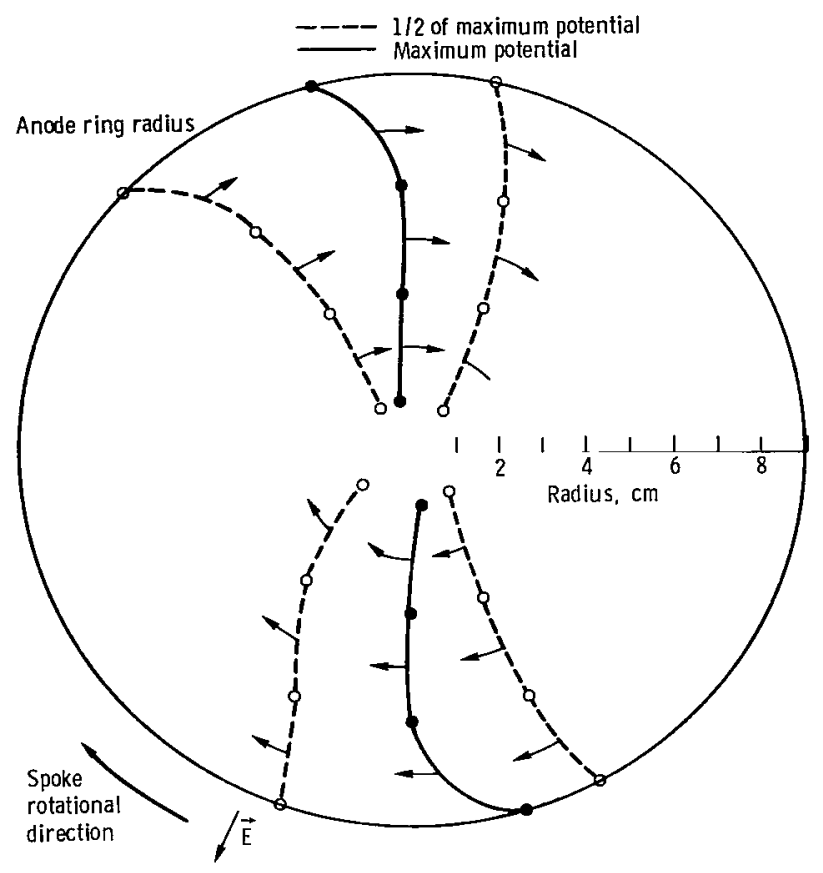

$$
\odot \vec{B}
$$

(a) Twelve positive midplane electrode rings; minor azimuthal mode, $m=-2$; background neutral gas (deuterium) pressure, $26 \times 10^{11}$ neutral particles $/ \mathrm{cm}^{3}$ (8. $0 \times 10^{-5}$ tor r); rotational frequency, 65 kilohertz.

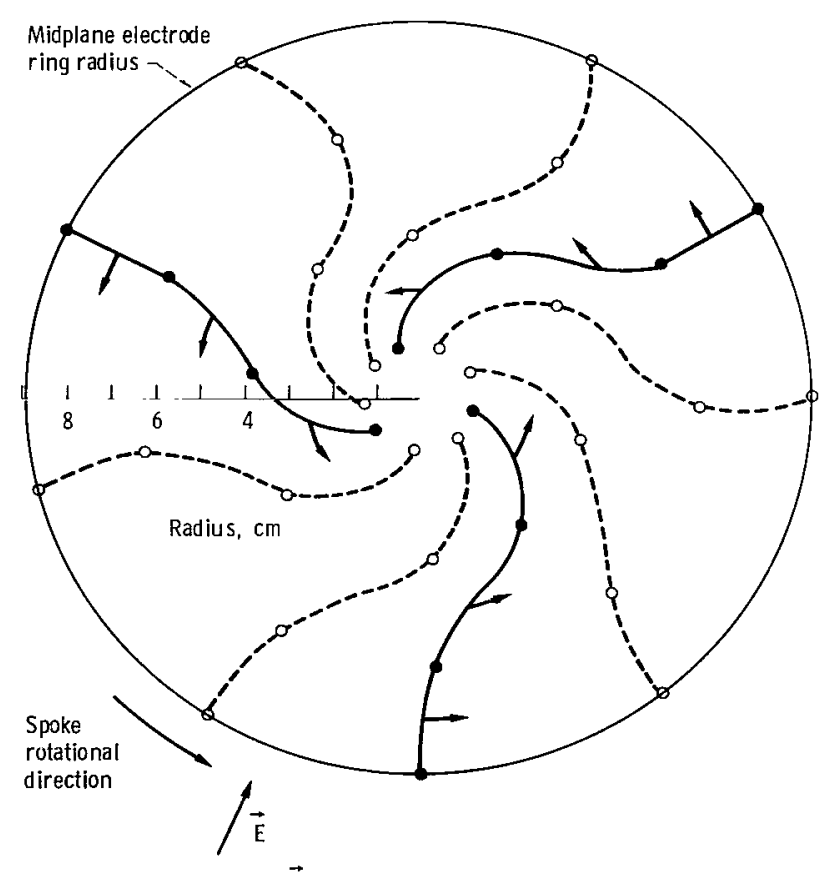

$\odot \vec{B}$

(b) Twelve negative midplane electrode rings; minor azimuthal mode number, $m=+3$, background neutral gas (deuterium) pressure, $13 \times 10^{11}$ neutral particles $/ \mathrm{cm}^{3}$ (4. $0 \times 10^{-5}$ torr); rotational frequency, 76 kilohertz.

Figure 22. - Rotating spoke structure in high-pressure mode of operation. Maximum magnetic field strength, 2.4 teslas; anode voltage, 2 kilovolts. 


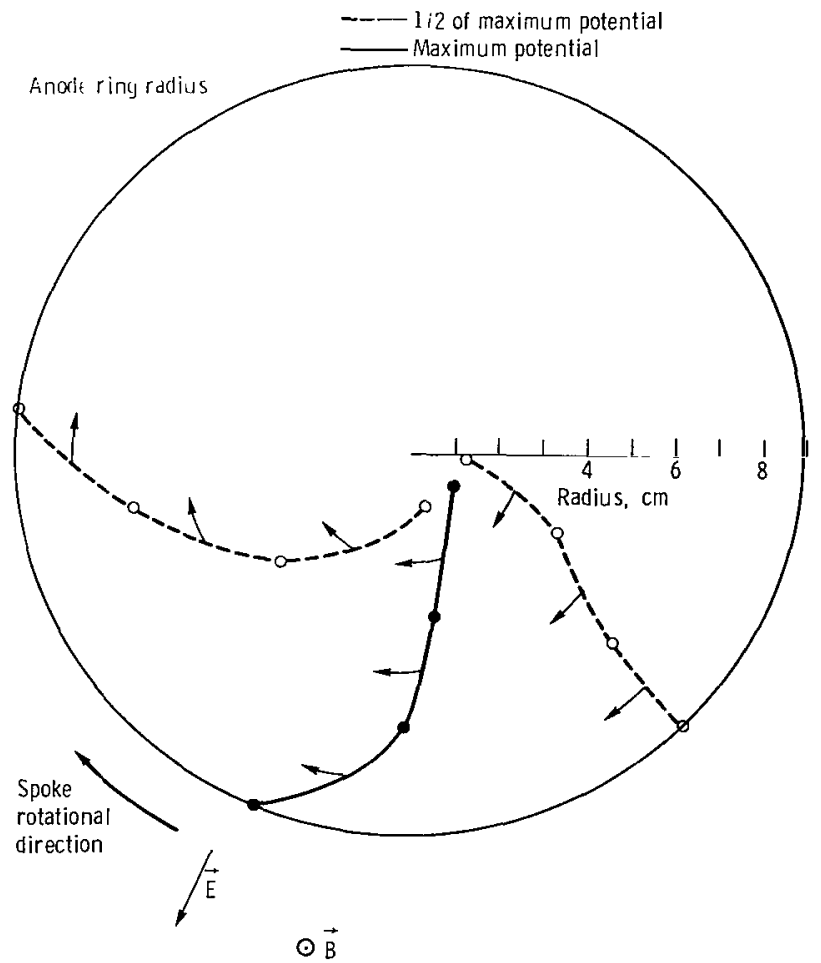

(a) Twelve positive midplane electrode rings; minor azimuthai mode, $m=-1$; rotational frequency, 14.5 kilohertz.

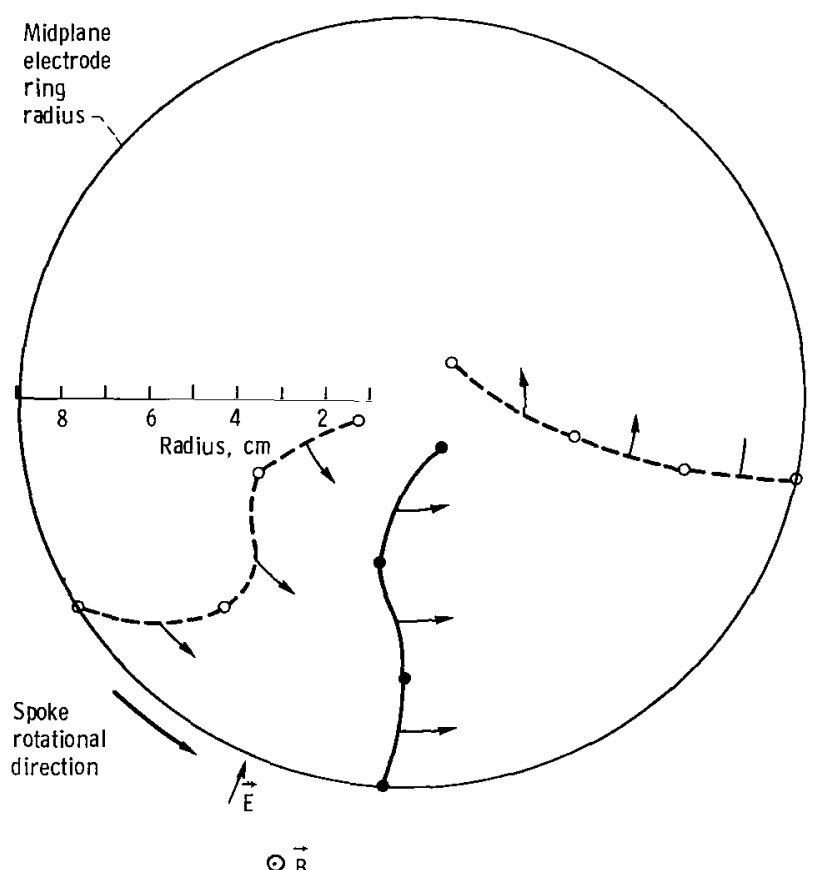

(b) Twelve negative midplane electrode rings; minor azimuthal mode, $m=+1$; $r$ tational frequency, 37 kilohertz.

Figure 23. - Rotating spoke structure in low-pressure mode. Maximum magnetic field strength. 2.4 teslas; background neutral gas (deuterium) pressure,

$17 \times 10^{11}$ neutral particles $/ \mathrm{cm}^{3}\left(5.3 \times 10^{-6}\right.$ torr); anode voltage, 2 kilovolts. 
situation existing in the high-pressure mode shown in figure 22.

The rotational frequencies of the spokes shown in figure 23 were very low, and it is questionable whether these are true $(\vec{E} \times \vec{B}) / B^{2}$ driven rotating spokes of the kind that exist in the high-pressure mode of operation. The data shown in figures 22 and 23 give a useful physical picture of the nature of the rotating spokes, but this picture has serious limitations. These data refer to the amplitudes of electrostatic potential fluctuations and do not necessarily reflect the radial or azimuthal density profiles of the plasma. These data do suggest, however, that substantial perturbations of density may occur in the bumpy-torus plasma.

\section{Parametric Behavior of Rotational Mode Number of Spokes}

The array of probes around the major circumference of the torus, shown in fig ure 18, was used to determine the major azimuthal mode number $l$, which describes the twisting of the disturbances around the major circumference of the torus from one sector to another, and the minor azimuthal mode number $\mathrm{m}$, which describes the number of spokes existing around the minor azimuth in the midplane of a given sector of the torus. The major azimuthal mode number of the ion spokes was zero under all conditions of operation. The physical significance of this is that the rotating ion spokes extend around the entire major circumference of the torus and rotate in phase in the manner shown in figure 24. The direction in which these spokes rotate is determined by the sign of the radial electric fields, but they remain in phase around the major circumference of the plasma and in phase from sector to sector. Under conditions investigated thus far, there has been no tendency of the rotating ion spokes to twist in such a manner that the electrostatic potential phase lines become helical rather than $l=0$ as shown. The major azimuthal mode number was zero even when the plasma was heated with only a single anode ring in one sector of the plasma.

Although the ion spokes always rotated with a major azimuthal mode number of zero, the electron spokes (discussed in detail in ref. 12) were observed to be incoherent from sector to sector. The electron spokes appeared to be driven by radial electric fields localized in the sheaths between the midplane electrode rings and the plasma. Since these radial electric fields were opposite to those that existed between the plasma and the grounded walls (fig. 7), the electron spokes rotated in the direction opposite to that of the ion spoke. Perhaps they were incoherent from sector to sector because the electrode ring sheaths were limited to the midplane of each sector.

The minor azimuthal mode number $m$ was measured with the array of probes shown in figure 18 over a wide range of plasma operating conditions. The minor mode behavior of the plasma is shown on the neutral gas pressure - anode voltage plane in figures $25(\mathrm{a})$ and (b) for 12 positive and 12 negative midplane electrode rings, respec- 

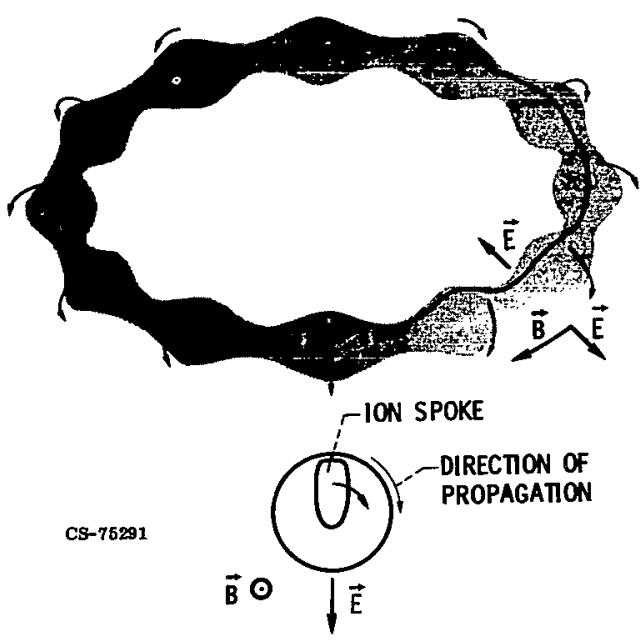

(a) With positive midplane electrode rings.
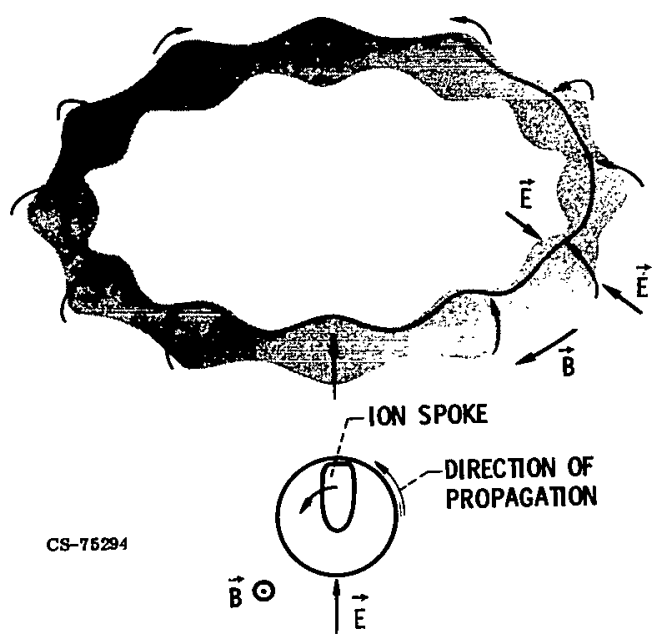

(b) With negative midplane electrode rings.

Figure 24. - Illustration of coherence of ion spoke around major circumference of torus and its direction of rotetion.

tively. With positive midplane electrodes (fig. 25(a)) there was a mode transition from $\mathrm{m}=-1$ to $\mathrm{m}=-2$ at approximately a 10 -kilovolt anode voltage. This is the same anode voltage for which there is a knee in the current-voltage curves shown in figure $4(\mathrm{a})$. In the high-pressure mode of operation, the plasma tended to operate in the $\mathrm{m}=-1$ mode at all but the lowest anode voltages. With 12 negative midplane electrode rings (fig. 25(b)) the mode structure is more complicated. In the high-pressure mode of operation, the mode numbers became smaller as the anode voltage increased; but in the low-pressure mode of operation, the higher mode numbers are associated with the higher voltages. 


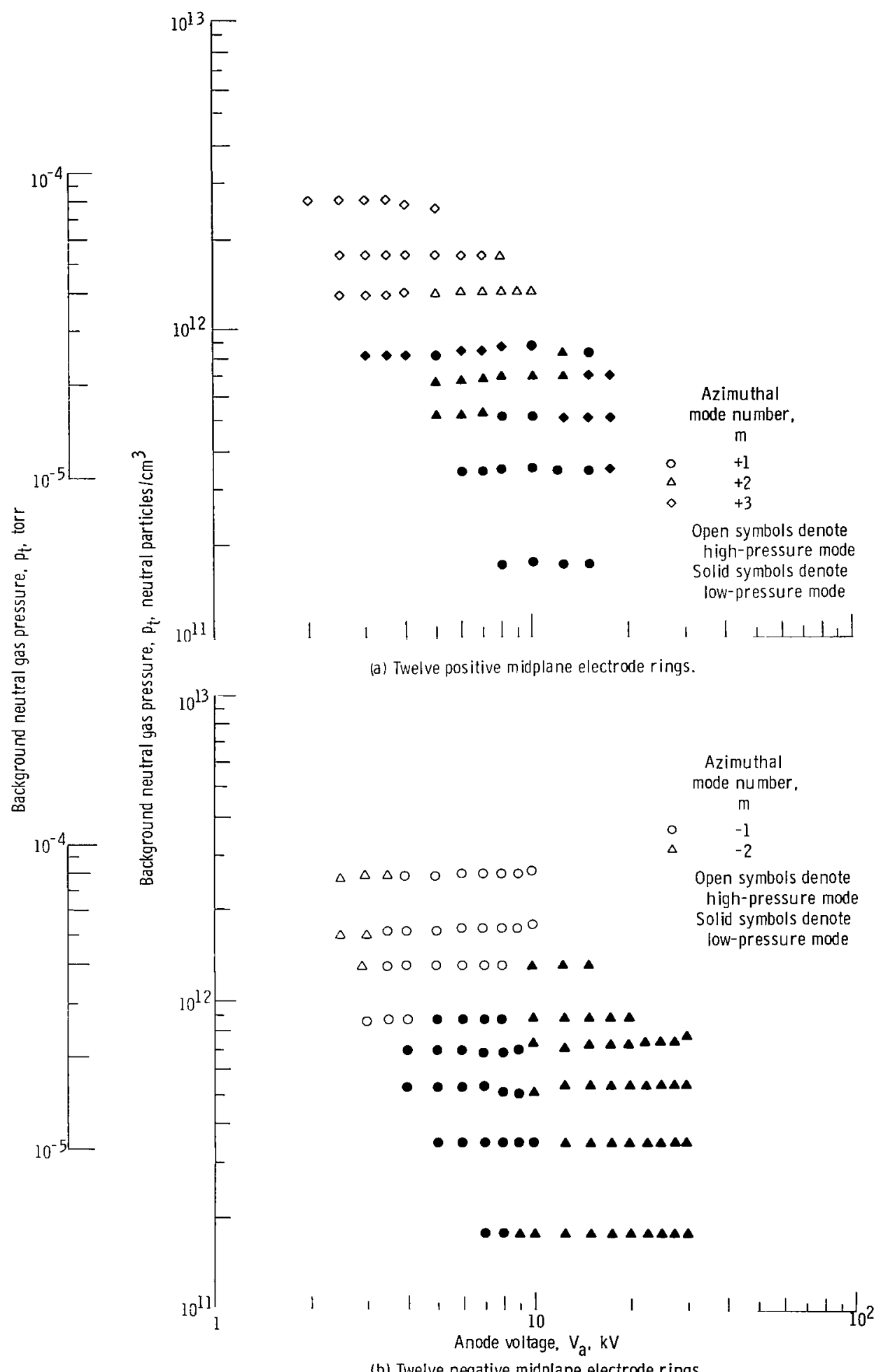

(b) Twelve negative midplane electrode rings.

Figure 25. - Minor mode behavior of plasma in anode voltage - background pressure plane. Maximum magnetic field strength, 2.4 teslas; neutral background gas, deuterium. 


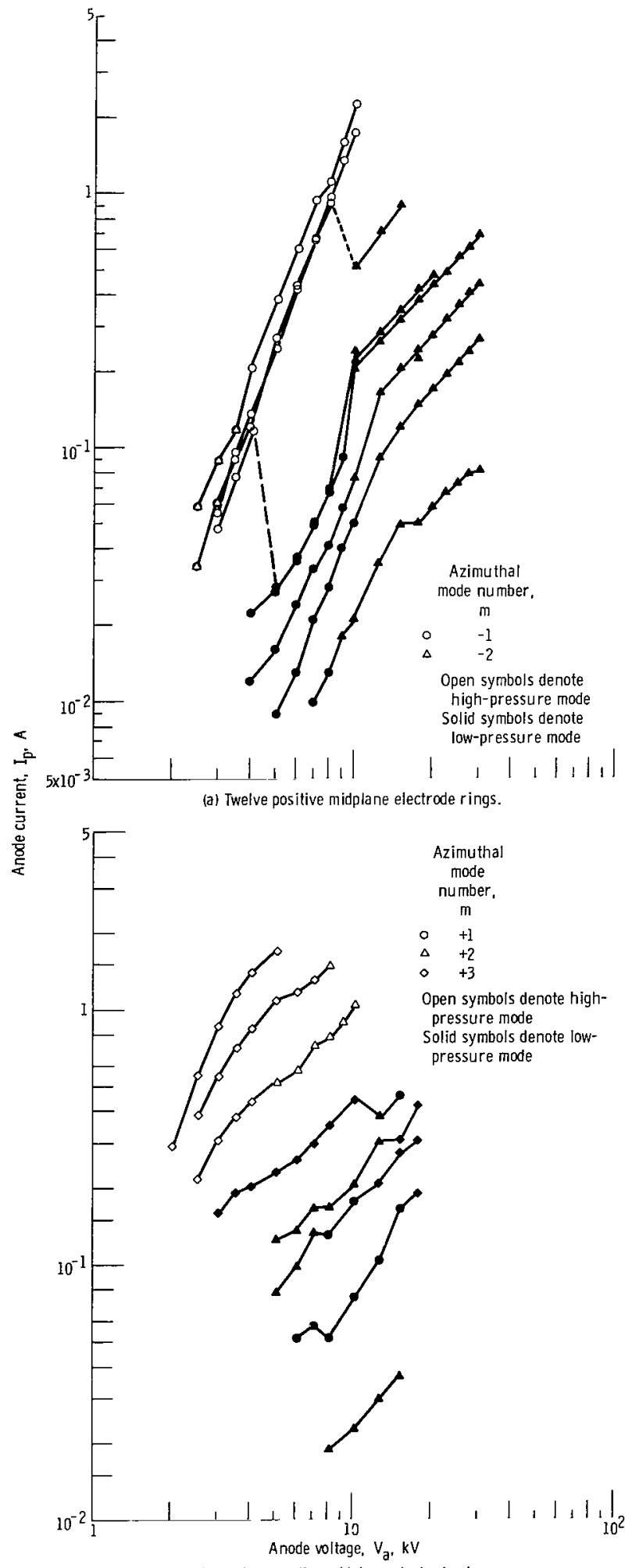

(b) Twelve negative midplane electrode rings.

Figu re 26. - Minor mode behavior oi plasma in anode current - anode voltage plane. Maximum magnetic field strength, 2.4 teslas; neutral background gas, deuterium. 
The minor azimuthal mode structure is indicated on a current-voltage diagram in figures 26(a) and (b) for 12 positive and 12 negative midplane electrode rings, respectively. In figure 26(a) there is a change in slope of the current-voltage curves at about a 10-kilovolt anode voltage where the minor azimuthal mode numbers change. In figure 26(b), the situation is more complicated although it does appear that the breaks in the current-voltage curve are associated with transitions in the minor azimuthal mode number.

\section{RELATION OF ION SPOKES TO ION HEATING}

The relation between ion heating and spoke velocities has been investigated extensively in the pilot-rig experiment (refs. 6, 7, 26, and 27). In these papers it was shown that the ion kinetic temperature, measured by a charge-exchange neutral analyzer, was proportional to the energy that an ion would acquire by moving with the $(\vec{E} \times \vec{B}) / B^{2} \mathrm{drift}$ velocity in the crossed electric and magnetic fields of the plasma. In references 6 and 7 , a relation between the ion kinetic temperature and the frequency of the ion spoke was derived and is

$$
\nu_{\mathrm{s}}=\frac{1}{4 \pi \mathrm{R}} \sqrt{\frac{\mathrm{e \textrm {T } _ { \mathrm { i } }}}{\mathrm{m}_{\mathrm{i}}}}
$$

where $R$ is the inner radius of the midplane electrode rings. Equation (3) was derived on the assumption that there is an equipartition of energy among the three degrees of freedom of ion motion and the spoke velocity and that the ion spoke velocity corresponds to the velocity of the most probable energy in a Maxwellian distribution of ion energy. This relation holds for the pilot-rig data reported in references 6 and 7, and it was considered of interest to find out whether the same physical processes are responsible for heating ions in the bumpy-torus plasma. The ion kinetic temperatures were measured with a charge-exchange neutral analyzer aimed across the plasma diameter. Particulars of this diagnostic technique may be found in references 1, 27, and 28. The ion kinetic temperature data were not used in these investigations when the ion distribution function departed significantly from a Maxwellian. The rotational frequency of the ion spoke was obtained from azimuthal probes such as those shown in figure 18, and in each case the rotational frequency per spoke was used as input data.

Figures 27(a) and (b) show the rotational frequency per spoke as a function of the observed ion kinetic temperature in electron volts. The solid and dashed lines show the values calculated from equation (3) for atomic deuterium ions and for molecular deuterium ions, respectively. For the 12 positive midplane electrode rings, some of the 


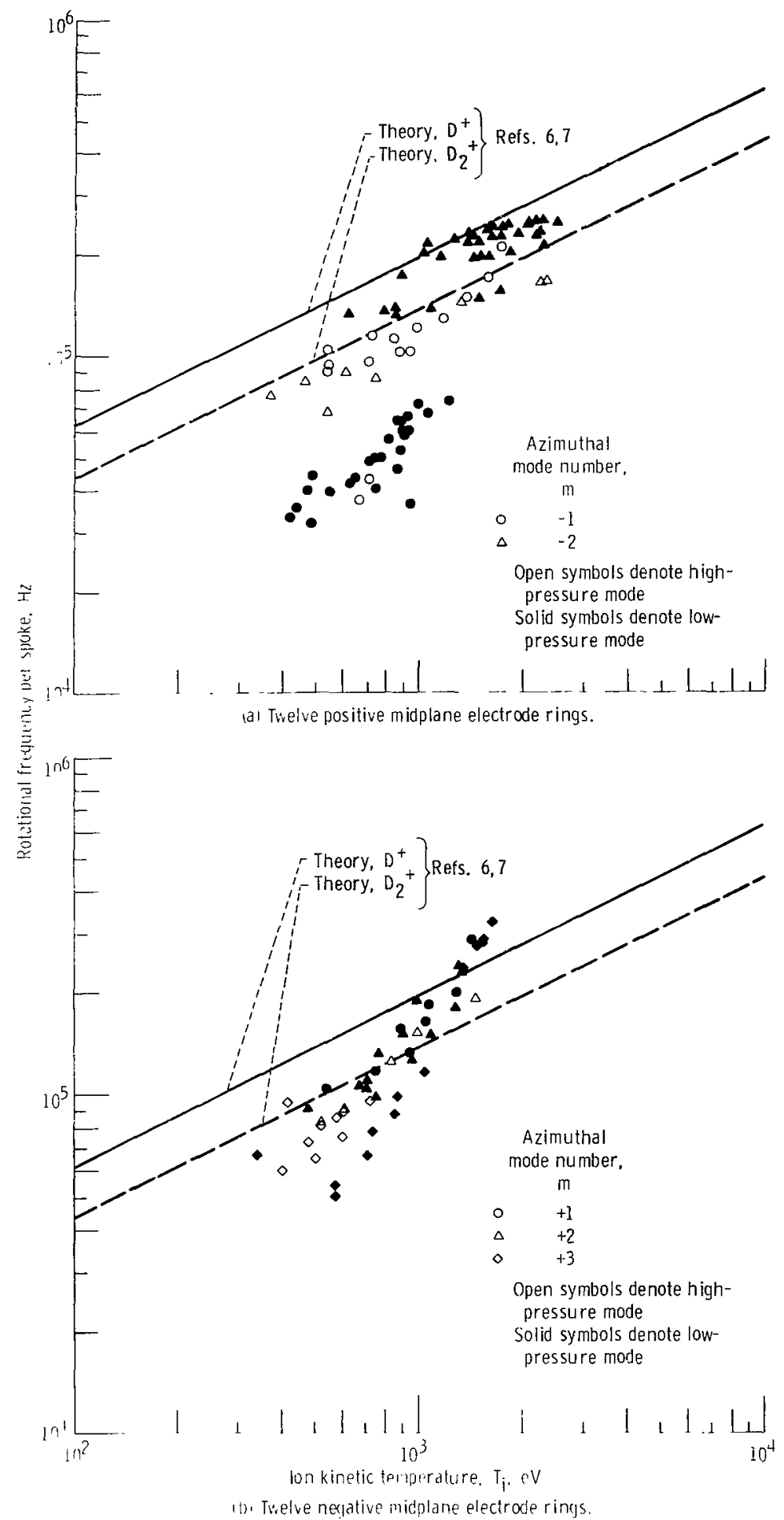

Fiujure 27. - Ion spoke rotational frequency per spoke as function of ion kinetic ter)perature for constant maximum magnetic field strenyth of 2.4 teslas. Neutral background gas, deuterium. 
data tend to fall along the calculated curve. Unlike the earlier mirror machine data, however, there is a substantial body of data at low frequencies, below about 60 kilohertz and $1 \mathrm{keV}$, that differ systematically from the values calculated by using this model. These anomalous low-pressure-mode data correspond to anode voltages below 10 kilovolts on the current-voltage curve of figure 4(a). The low-pressure-mode data above 10 kilovolts and with a mode number of $m=-2$ agree with the model. This suggests that below 10 kilovolts the anomalous rotating spokes are unrelated to the ion kinetic temperature and that ions acquire energy by some physical process other than being accelerated to the velocity of the rotating spoke. Some of the high-pressure-mode data also fall substantially below the calculated curves, although these data approximate a straight-line slope of $1 / 2$. However, most of the high-pressure-mode data lie near the molecular deuterium ion curve and are at a lower frequency than the high-voltage, lowpressure-mode data.

The data shown in figure 27(b) for the 12 negative midplane electrodes fall in the general vicinity of the model predictions, but the agreement is not good. There is some tendency for the data to fall along a straight line of slope other than $1 / 2$, which would indicate that this simple model does not include all of the important physical processes by which ions gain energy.

The ion kinetic temperature and the ion spoke rotational frequency were measured as the magnetic field was varied from 0.24 to 2.4 teslas. These data are shown in figures 28(a) and (b) for 12 positive and 12 negative midplane electrode rings, respectively. The data for the positive midplane electrode rings (fig. 28(a)) fall quite close to the model prediction over a factor of 10 in magnetic field strength. There are two discrepant points at $600 \mathrm{kilohertz}$ that may be misidentified electron spokes, and there is one point at approximately $48 \mathrm{kilohertz}$ that may be in the anomalous low - frequency mode shown in figure 27(a). For the 12 negative midplane electrode rings (fig. 28(b)), the data points for both the high- and low-pressure modes of operation lie substantially above the model prediction, with the highest points representing the lowest magnetic field strengths. These negative electrode data appear to have little or no correlation with the simple rotating spoke model.

The plasma could be heated with only a single positive anode ring energized. Data were taken when only the midplane electrode in sector 9 was energized with positive voltage (fig. 29). This was the same sector in which the charge-exchange neutral analyzer was located. The high-pressure-mode data are in generally good agreement with the model prediction relating the spoke rotational frequency to the ion kinetic temperature. However, there is a cluster of data between 30 and 40 kilohertz in the lowpressure mode that do not agree with the prediction in terms of both magnitude and functional dependence. Data similar to figure 29 could not be taken with one negative midplane electrode, because a plasma could not be generated with such a configuration. Figure 30 shows the same data at a constant magnetic field with only the midplane elec- 


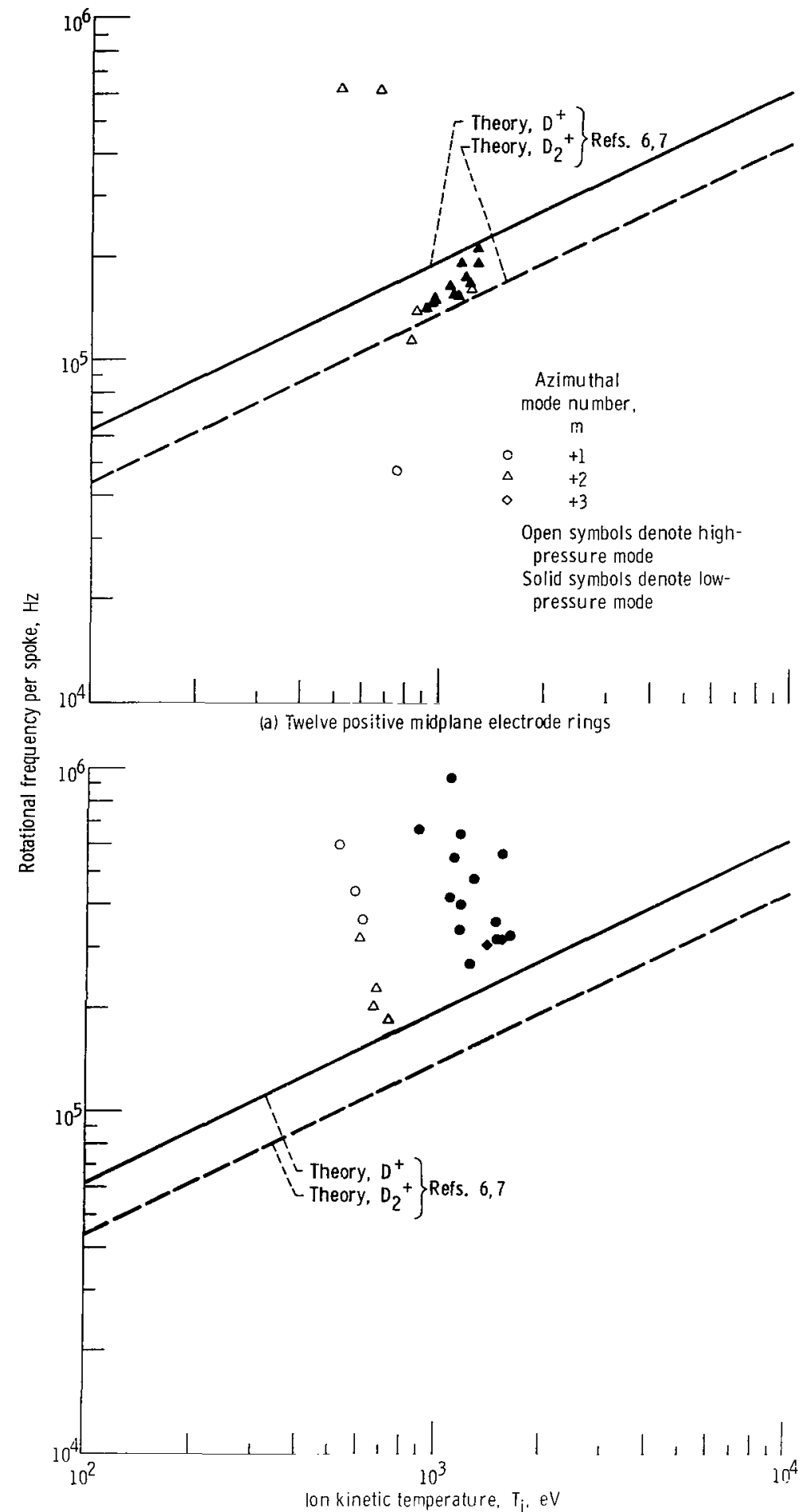

(b) Twelve nexative midplane electrode rings.

Figure 28. - Ion spoke rotational frequency per spoke as function of ion kinetic teperature for maximum magnetic fieid strength varying from $0.2+$ to $2 .+$ teslas. Neu tral background gas, deuterium. 


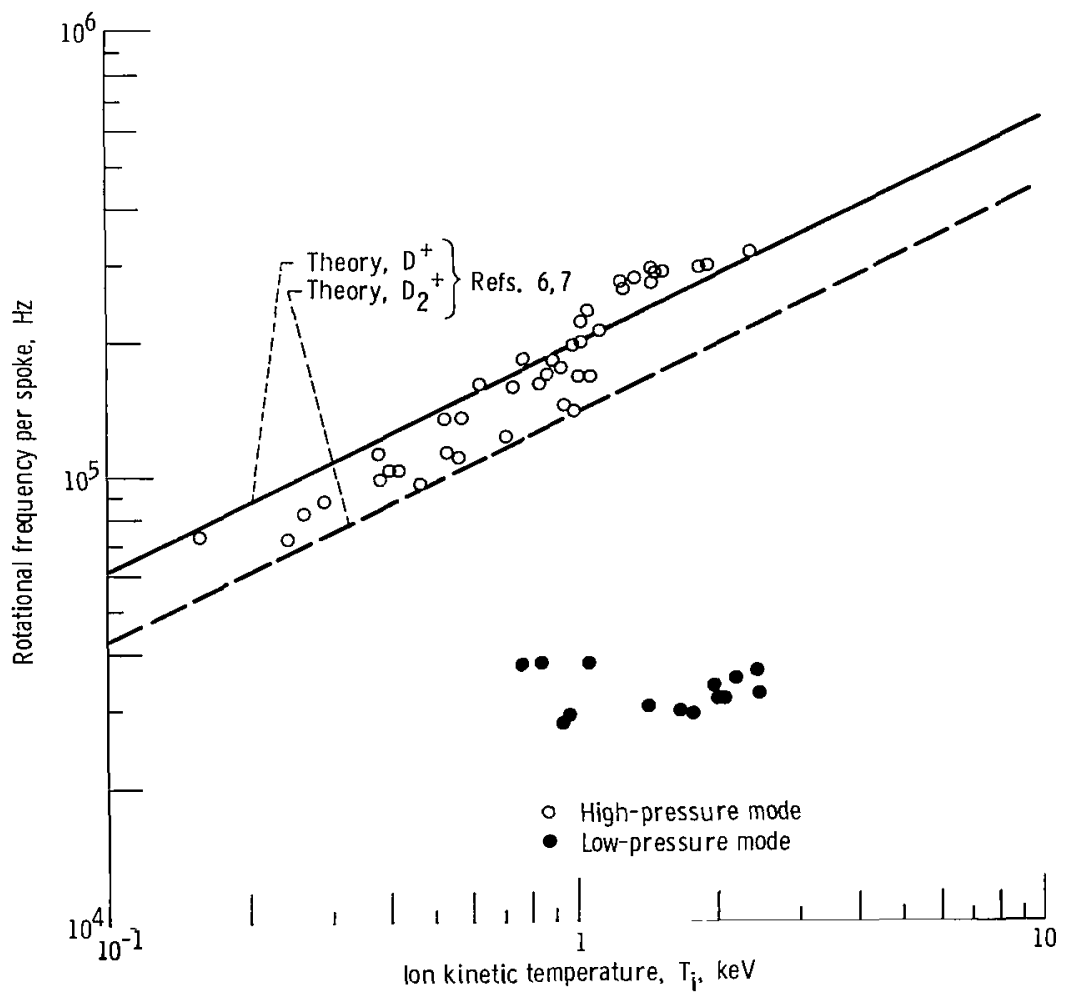

Figure 29. - Ion spoke rotational frequency per spoke as function of ion kinetic temperature for plasma generated with a single positive midplane electrode ring located in sector 9 of the torus. Maximum magnetic field strength, 2.4 teslas; neutral background gas, deuterium.

trode ring in sector 3 energized to positive potentials. This sector was at the opposite diameter of the torus from the charge-exchange neutral analyzer. In this case also, the high-pressure-mode data were in generally good agreement with the model prediction, but the low-pressure-mode data were substantially below the prediction, although possibly lying on a trend line with the predicted slope of $1 / 2$.

From these data is appears justified to conclude that, with positive potentials on the midplane electrode rings, there is a correlation between ion temperature and ion spoke rotational frequency that is given by the same physical model as was previously found to apply in the mirror machine pilot-rig experiment (refs. 6, 7, 26, and 27). An exception occurs when the positive midplane electrode ring voltage is in that region of parameter space below an anode voltage of 10 kilovolts and in the low-pressure mode where the current-voltage curves of figure 4 (a) have a slope of approximately 2. 0.

The agreement of the data with the simple model of equation (3) was generally poor when the plasma was biased to high negative potentials with negative midplane electrodes. The experimental data lie in the general vicinity of the model predictions, but it is not clear that the data have the functional dependence predicted. 


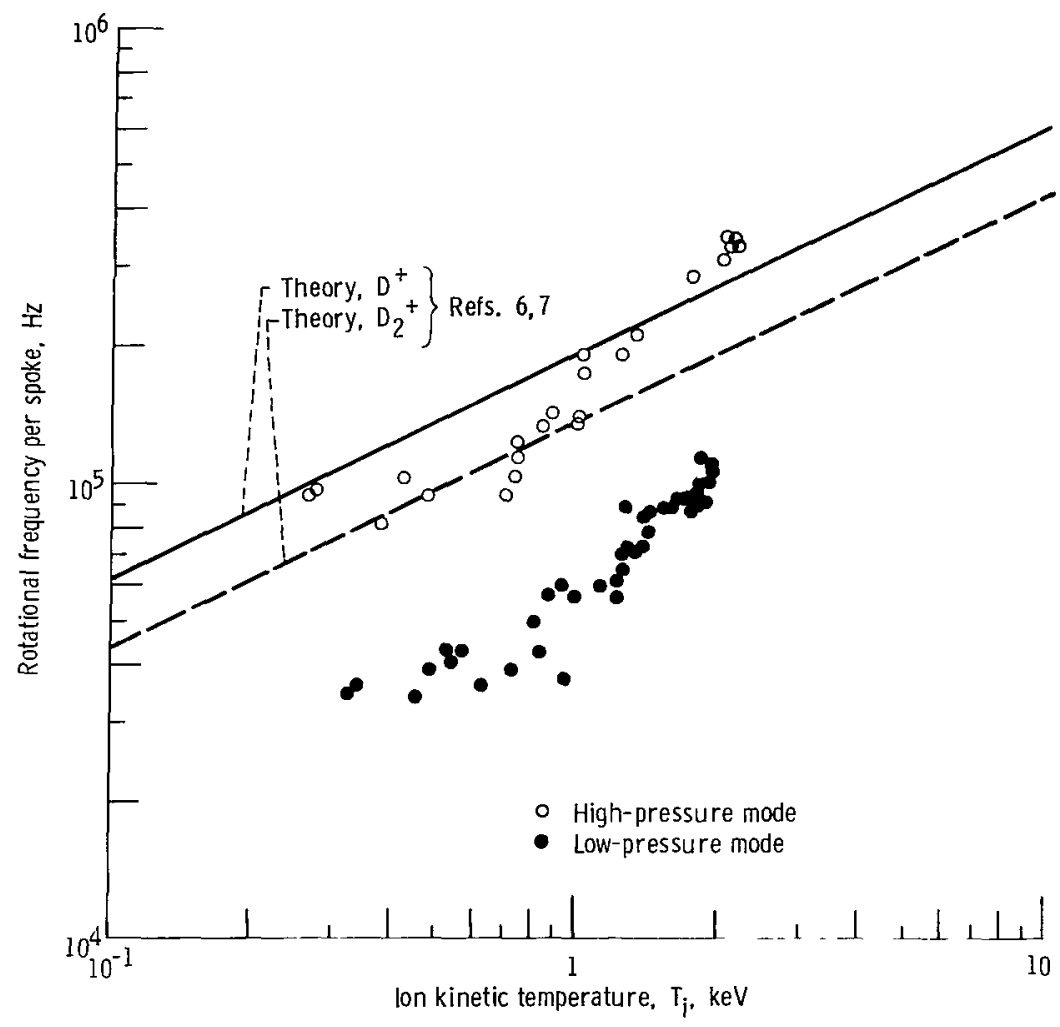

Figure 30. - Ion spoke rotational frequency per spoke as function of ion kinetic temperature for plasma generated with a single positive midplane electrode ring located in sector 3 of the torus. Maximum magnetic field strength, 2.4 teslas; neutral background gas, deuterium.

\section{FACTORS AFFECTING ION HEATING}

As previously stated, a plasma could be generated with fewer than 12 midplane electrode rings. The electrode rings not energized with high voltage were in all cases retracted from the plasma volume. When negative potentials were applied to the midplane electrode rings, no plasma whatever was generated by using a single negative electrode ring. When positive potentials were applied to the midplane electrode rings, a plasma could be generated with as few as one anode ring.

The effect of the number of anode rings on ion kinetic temperature was determined with the arrangement shown in figure 31. After taking ion temperature data with 12 positive and with 12 negative midplane electrode rings, ion temperatures were measured when only a single positive anode ring was used. The ion energy distribution functions were measured with a charge-exchange neutral analyzer in the manner described in references 1,27 , and 28 . The plasma was generated by an anode ring in the sector in which the neutral particle analyzer was located, and it was also generated with the anode 


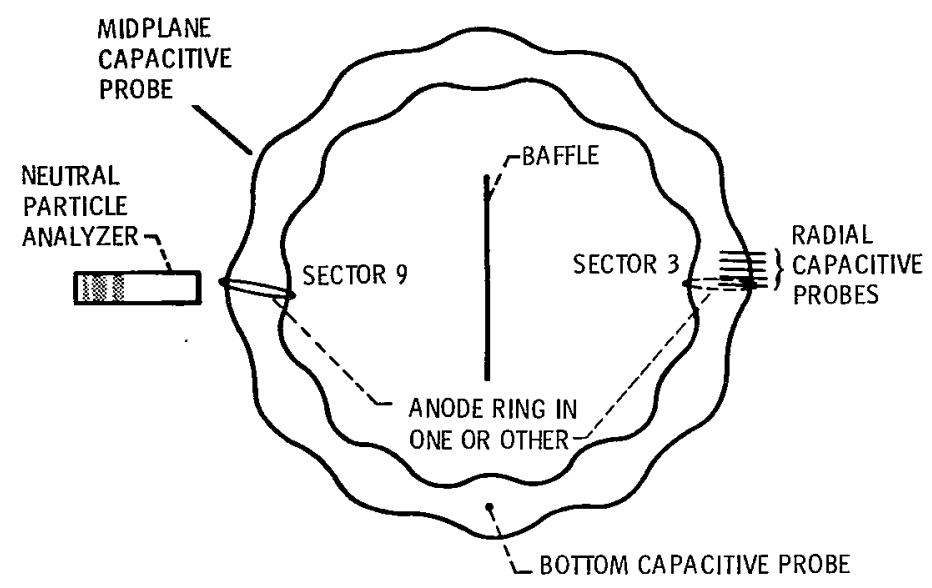

Figure 31. - Position of charge-exchange neutral particle analyzer and two single anode rings that were used one at a time to generate and heat the plasma.

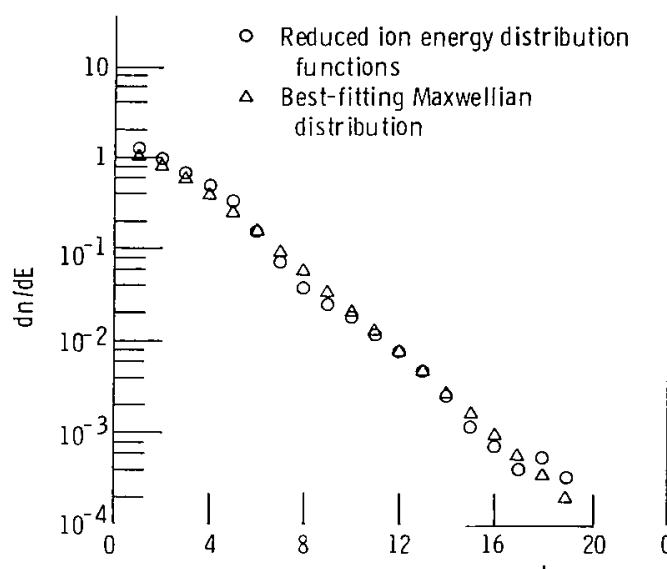

(a) Single positive anode ring in same sector as neutral particle analyzer, sector 9 . Best-fitting ion kinetic temperature, $\mathrm{T}_{\mathbf{j}}$. $1.81 \mathrm{keV}$.

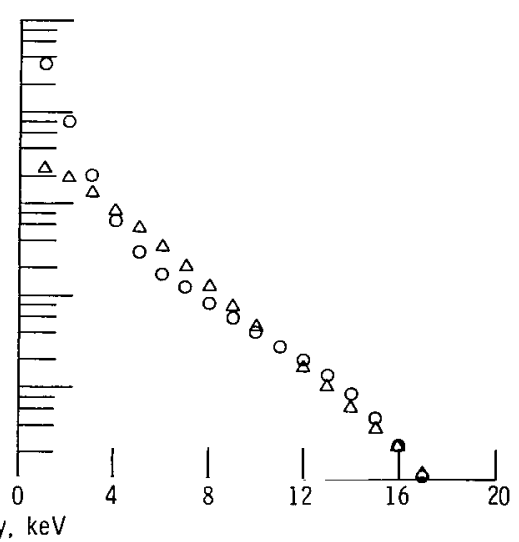

Figure 32. - Reduced ion energy distribution functions and best-fitting Maxwellian distributions measured when the plasma was generated by a single anode ring.

ring located in sector 3 across the major diameter of the torus from the neutral particle analyzer. During these latter measurements, a metallic baffle plate was installed on the major axis of the tank to prevent charge-exchange neutrals originating at the opposite side of the torus from reaching the neutral particle analyzer.

When the midplane electrode rings were in sector 3 or 9 , data were taken at the same conditions of magnetic field, neutral background pressure, and anode voltage. Some characteristic results of these measurements are shown in figure 32 . In figure $32(\mathrm{a})$ is the ion energy distribution function observed when the anode ring and the 
neutral particle analyzer were both in sector 9 of the torus. The open circles are the reduced experimental data, and the triangles are the best-fitting Maxwellian distribution with a kinetic temperature of 1.8 kilovolts. In this case, the ion energy distribution function is very Maxwellian. In figure $32(\mathrm{~b})$ is the distribution function taken for the same operating conditions but with the single anode ring located at the opposite side of the torus in sector 3 . In this case, the ion energy distribution function is less Maxwellian than in the case for which the anode ring and neutral particle analyzer were in the same sector, but the best-fitting ion kinetic temperature in this case is 1.5 kilovolts. Under a wide range of operating conditions, the ion distribution function was more nearly Maxwellian when the anode ring and neutral particle analyzer were in the same sector than when the plasma was generated by an anode ring at the opposite sector of the plasma.

Figure 33 compares the ion kinetic temperatures observed when only anode 3 was energized with the ion kinetic temperatures observed when only anode 9 was energized.

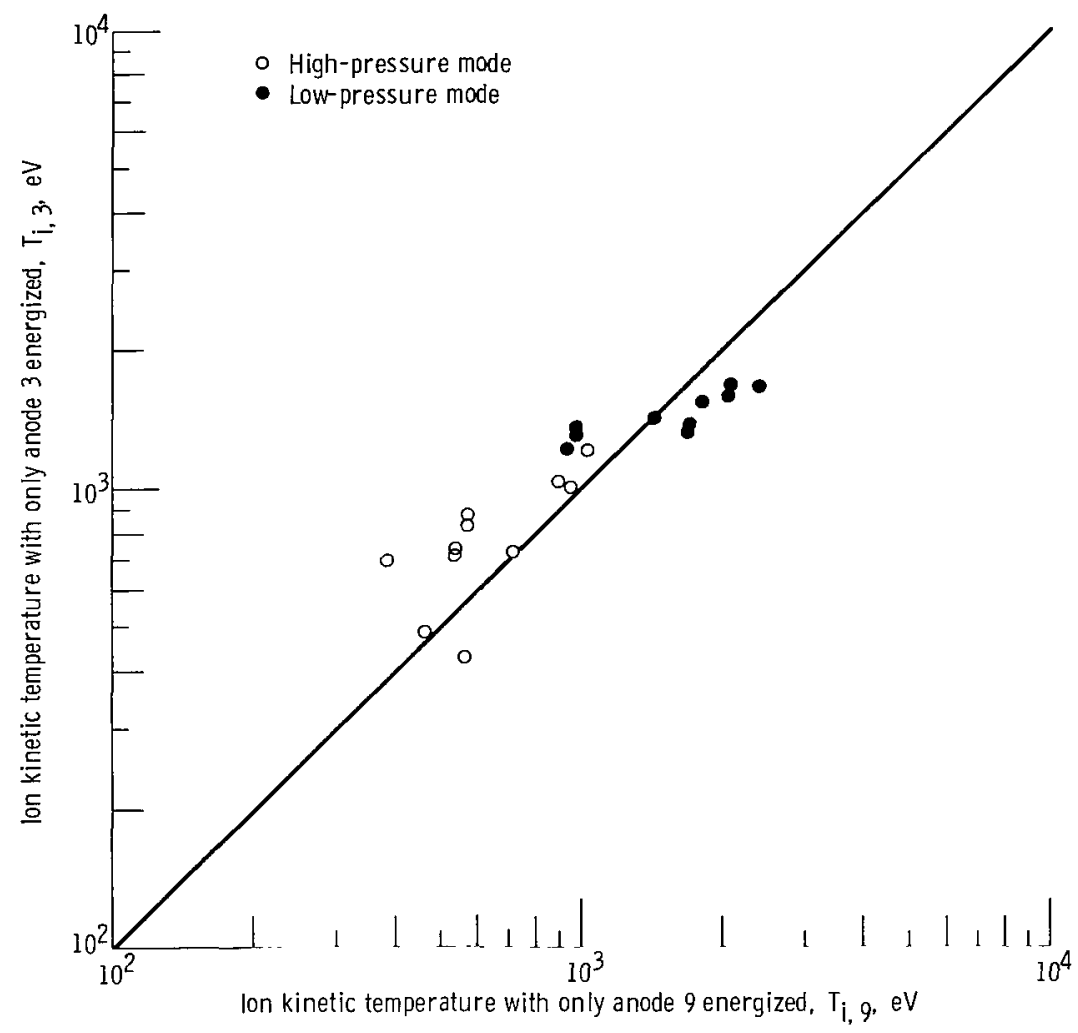

Figure 33. - Ion kinetic temperature measured with only anode 3 energized, compared with ion kinetic temperatures measured under identical operating conditions with only anode 9 energized. 
The comparative data were taken under identical operating conditions of magnetic field, neutral background pressure, and anode voltage. The high-and low-pressure modes of operation are shown by the open and solid circles, respectively. The data tend to lie along the line of agreement, showing that there is substantial agreement in the ion kinetic temperatures achieved whether the anode is located in the nearest sector or at the opposite diameter of the plasma torus. This result demonstrates that a single anode ring is capable of heating ions about equally well around the entire plasma torus. The energetic ions can enter and be detected in the neutral particle analyzer only if they have very small velocities parallel to the magnetic field. Thus, the ions detected in sector 9 at the neutral particle analyzer position must have originated in that sector.

In this steady-state discharge, we can obtain a very simple approximate expression for the ion heating efficiency. The efficiency is defined as the power deposited in the ion population divided by the total power into the plasma. The power into the ion population may be written

$$
\mathrm{W}_{\mathrm{i}}=\frac{\mathrm{en}_{\mathrm{i}} \mathrm{T}_{\mathrm{i}} \mathrm{V}_{\mathrm{p}}}{\tau_{\mathrm{e}}}
$$

where $\tau_{e}$ is the energy confinement time of the plasma. If we wish a lower bound on the power into the ion population and ignore the energy carried away by energetic charge-exchange neutrals, we can replace the energy confinement time by the ion confinement time $\tau_{\mathrm{c}}$. However, the current flowing to the plasma is just equal to

$$
\mathrm{I}_{\mathrm{A}}=\frac{\mathrm{en}_{\mathrm{i}} \mathrm{V}_{\mathrm{p}}}{\tau_{\mathrm{c}}}
$$

If we further assume that all the current is ion current and that there are no parasitic currents flowing outside the plasma volume, the power into the plasma is equal to

$$
\mathrm{W}_{\mathrm{A}}=\mathrm{V}_{\mathrm{A}} \mathrm{I}_{\mathrm{A}}
$$

Using equations (4) to (6), we may write the efficiency as

$$
\eta_{\mathrm{i}}=\frac{\mathrm{W}_{\mathrm{i}}}{\mathrm{W}_{\mathrm{A}}}=\frac{\mathrm{T}_{\mathrm{i}}}{\mathrm{V}_{\mathrm{A}}}
$$

which is numerically equal to the ratio of ion kinetic temperature in electron volts to the applied potential on the midplane electrodes in voits. In the bumpy-torus plasma, this estimate of heating efficiency is likely to be a lower bound. We know that there is a 
significant flux of charge-exchange neutrals to the walls, but there do not seem to be any significant parasitic currents that flow outside the plasma volume. Flow of current through the plasma as a result of electron emission from the cathode surfaces (refs. 9 and 10) is thought to be small but cannot be directly measured.

Figure 34 is a comparison plot of the ion heating efficiency, as defined in equation (7), with only anode 3 energized as a function of the ion heating efficiency observed

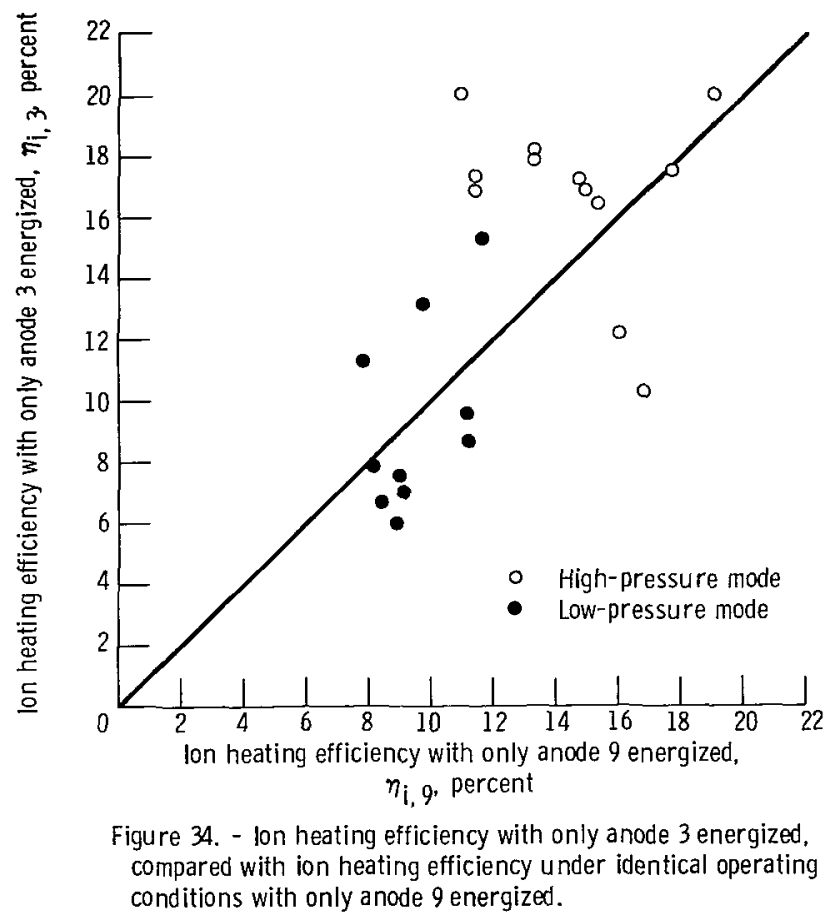

under identical operating conditions with only anode 9 energized. The scatter in the data is large, but no serious loss of efficiency seems to occur when the plasma is created by the anode ring opposite from the neutral particle analyzer. Even in this case, efficiencies as high as 20 percent result. Figure 35 compares the ion kinetic temperature observed with all 12 electrode rings positive with the ion kinetic temperature at the same operating conditions when only anode 9 was operated at positive potential. The ion kinetic temperatures lie along the line of agreement in the low-pressure mode of operation but appear to be somewhat higher in the high-pressure mode of operation when all 12 electrode rings are positive. Figure 36 compares the ion heating efficiency with all 12 electrode rings positive with the ion heating efficiency when only anode 9 was energized. The ion heating efficiencies were essentially the same in the low-pressure mode of operation, but the efficiencies were, also, substantially higher in the high-pressure mode when all 12 electrode rings were positive. 


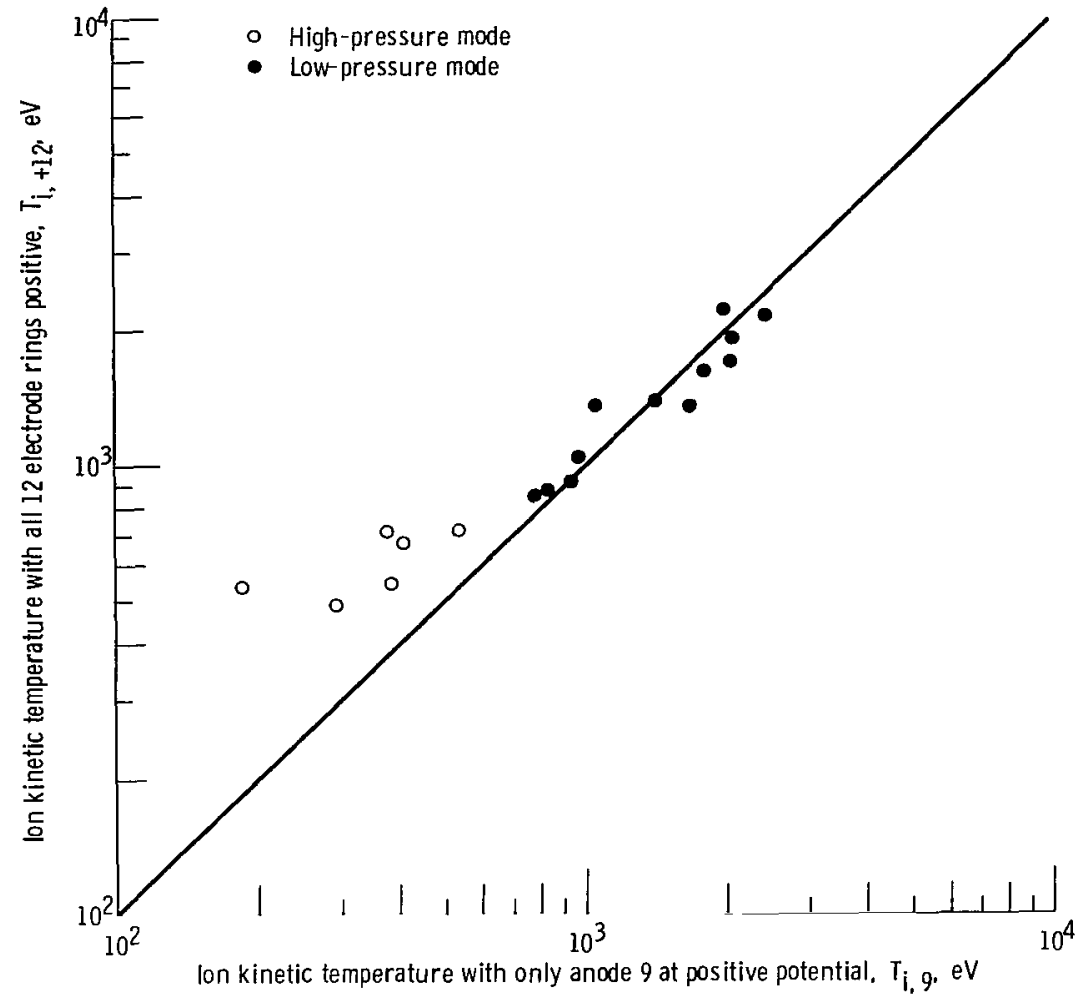

Figure 35. - Ion kinetic temperature with all 12 electrode rings positive, compared with ion kinetic temperature under identical operating conditions with only anode 9 energized.

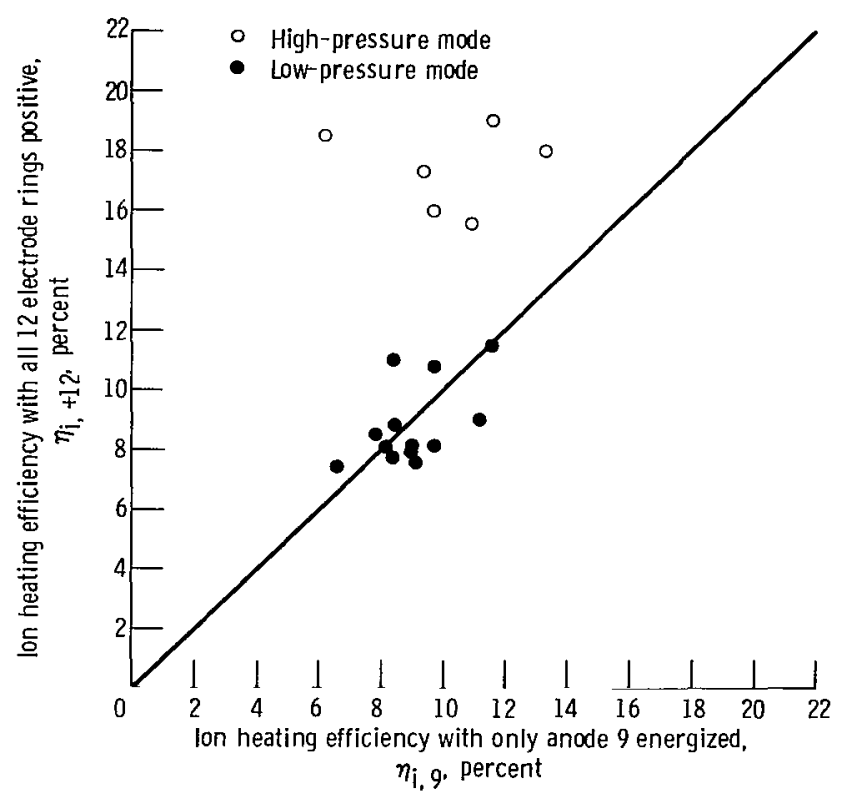

Figure 36. - Ion heating efficiency with all 12 electrode rings positive, compared with ion heating efficiency under identical operating conditions with only anode 9 energized. 


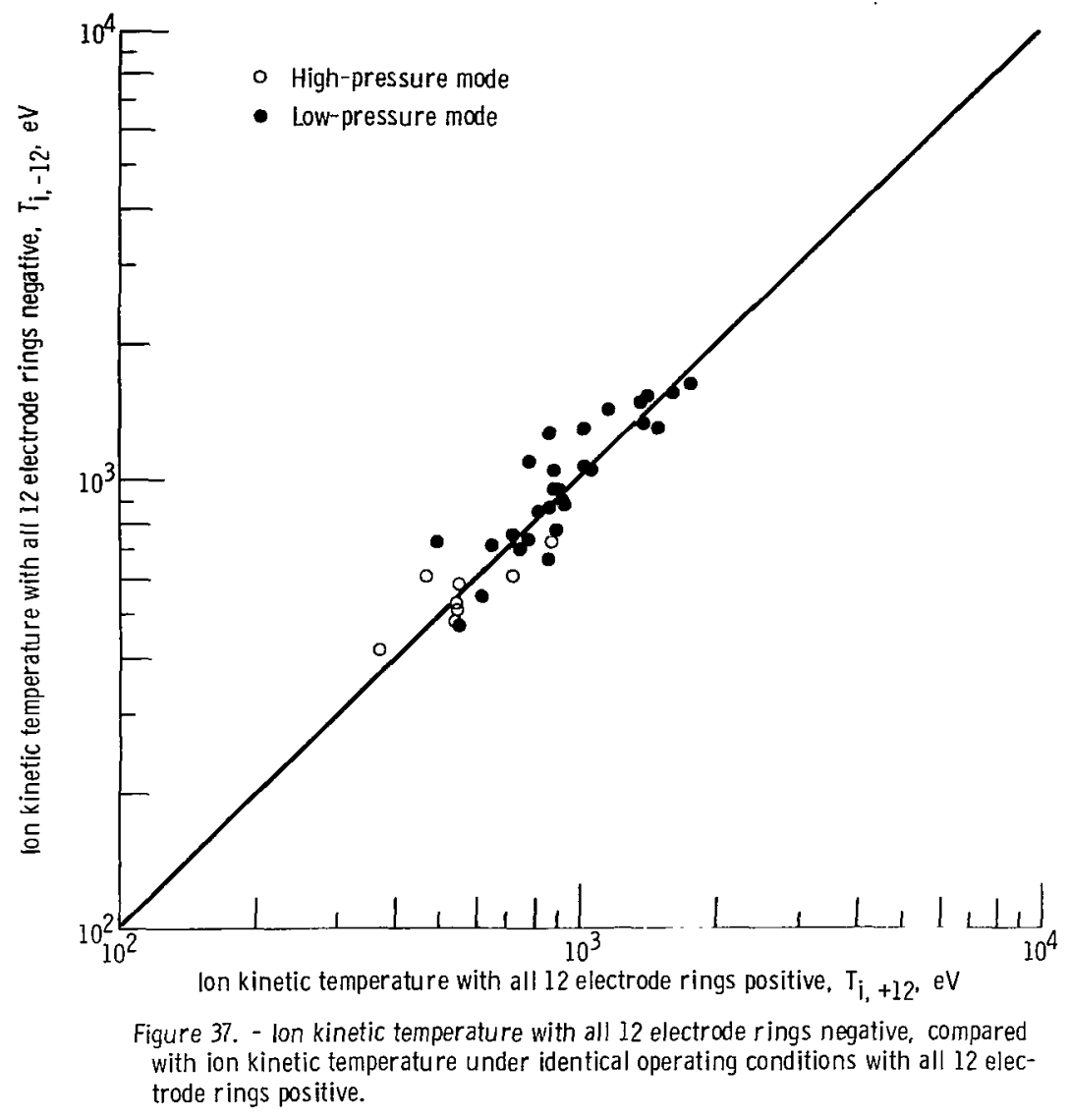

Two sets of ion kinetic temperature measurements were made under identical operating conditions of magnetic field, neutral background pressure, and anode voltage: one where all 12 midplane electrode rings were biased to positive potentials, and one set where the bias was negative. Figure 37 compares these data sets. In both the high- and low-pressure modes of operation, all data lie close to the line of agreement, implying that the ions can be heated equally well by biasing the plasma to positive or negative potentials. Figure 38 compares the ion heating efficiencies obtained with all 12 electrode rings positive and negative. The efficiencies are spread equally on either side of the line of agreement, and there does not seem to be any improvement in ion heating efficiency by biasing the midplane electrode rings to one sign or another of potential. It is evident in figure 38 , however, that the ion heating process is more efficient in the highpressure mode of operation than in the low-pressure mode of operation. In the lowpressure mode of operation, the efficiencies ranged from about 5 to 15 percent, whereas in the high-pressure mode of operation, the efficiencies ranged from 15 to 22 percent. 


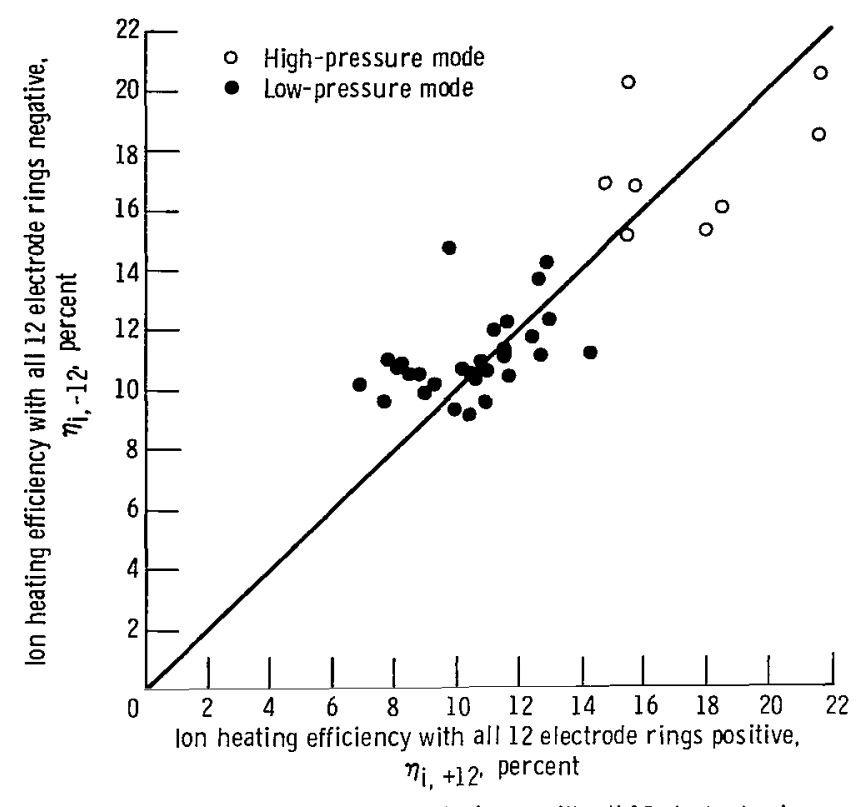

Figure 38. - Ion heating efficiency with all 12 electrode rings negative, compared with ion heating efficiency under identical operating conditions with all 12 electrode rings positive.

\section{PARAMETRIC VARIATIONS OF ION KINETIC TEMPERATURE}

\section{AND HEATING EFFICIENCY}

Investigating the dependence of ion kinetic temperature and heating efficiency on the operating parameters should lead to the most profitable method of achieving higher ion kinetic temperatures and higher ion heating efficiencies. Figures $39(\mathrm{a})$ and (b) show ion kinetic temperature as a function of anode voltage for three background neutral gas pressures with 12 positive and 12 negative midplane electrodes, respectively. In both cases the ion kinetic temperatures increased with increasing anode voltage, but at a somewhat less than linear rate. At a given anode voltage, higher ion kinetic temperatures were achieved by going to higher neutral background pressures of deuterium gas. The ion heating efficiencies for the same operating conditions are shown in figures 40 (a) and (b) for 12 positive and 12 negative midplane electrode rings, respectively. The ion heating efficiencies tended to decrease slowly as the anode voltage increased, but at a given anode voltage the efficiency increased as the neutral background pressure increased. In figure $40(\mathrm{~b})$ it is evident that with 12 negative midplane electrode rings, the efficiency is more nearly independent of the anode voltage and that the high-pressure mode of operation is more efficient than the low-pressure mode. 


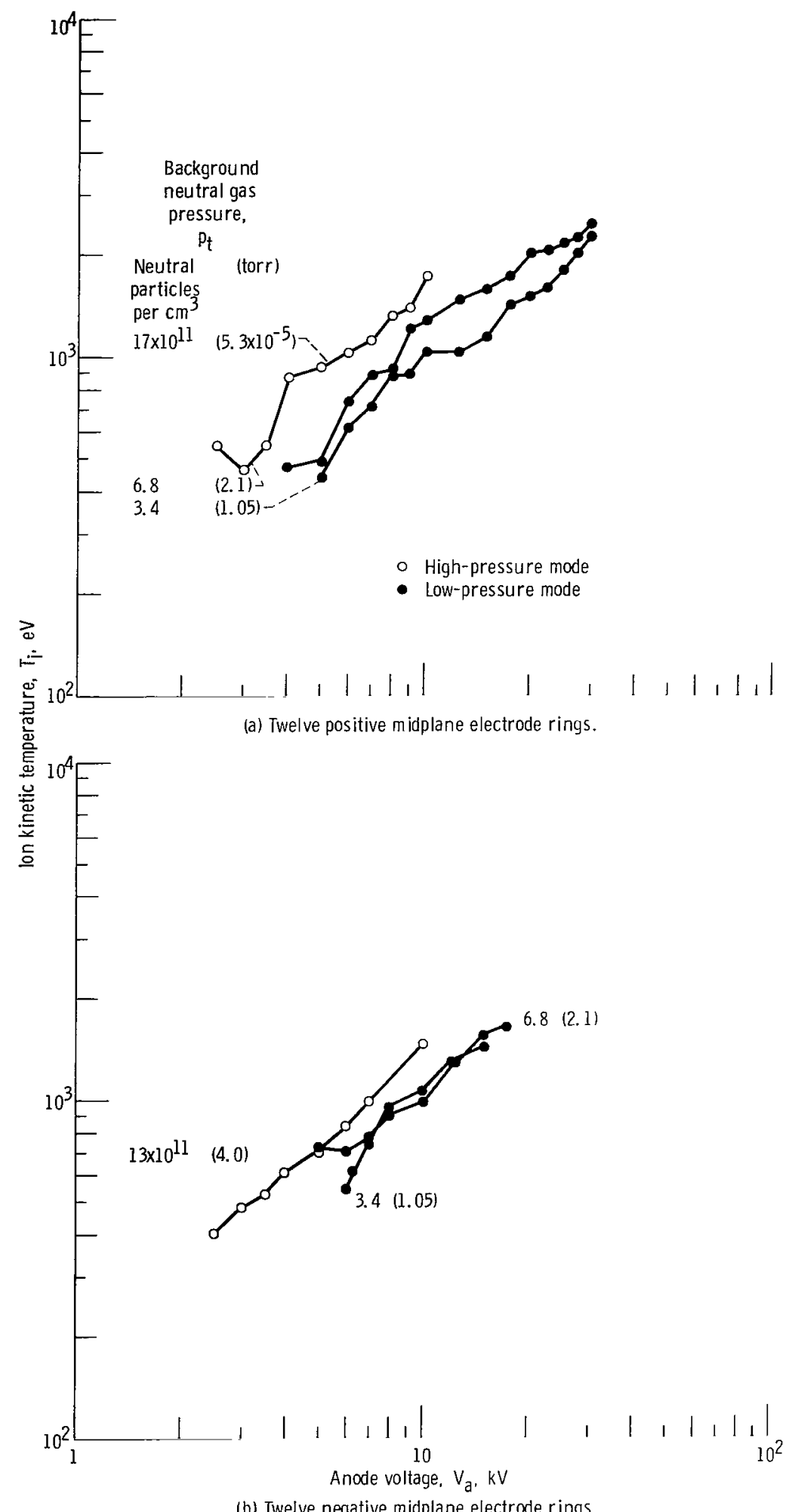

(b) Twelve negative midplane electrode rings.

Figure 39. - Ion kinetic temperature as function of anode voltage for three background pressures of deuterium gas. Maximum magnetic field strength, 2.4 teslas. 


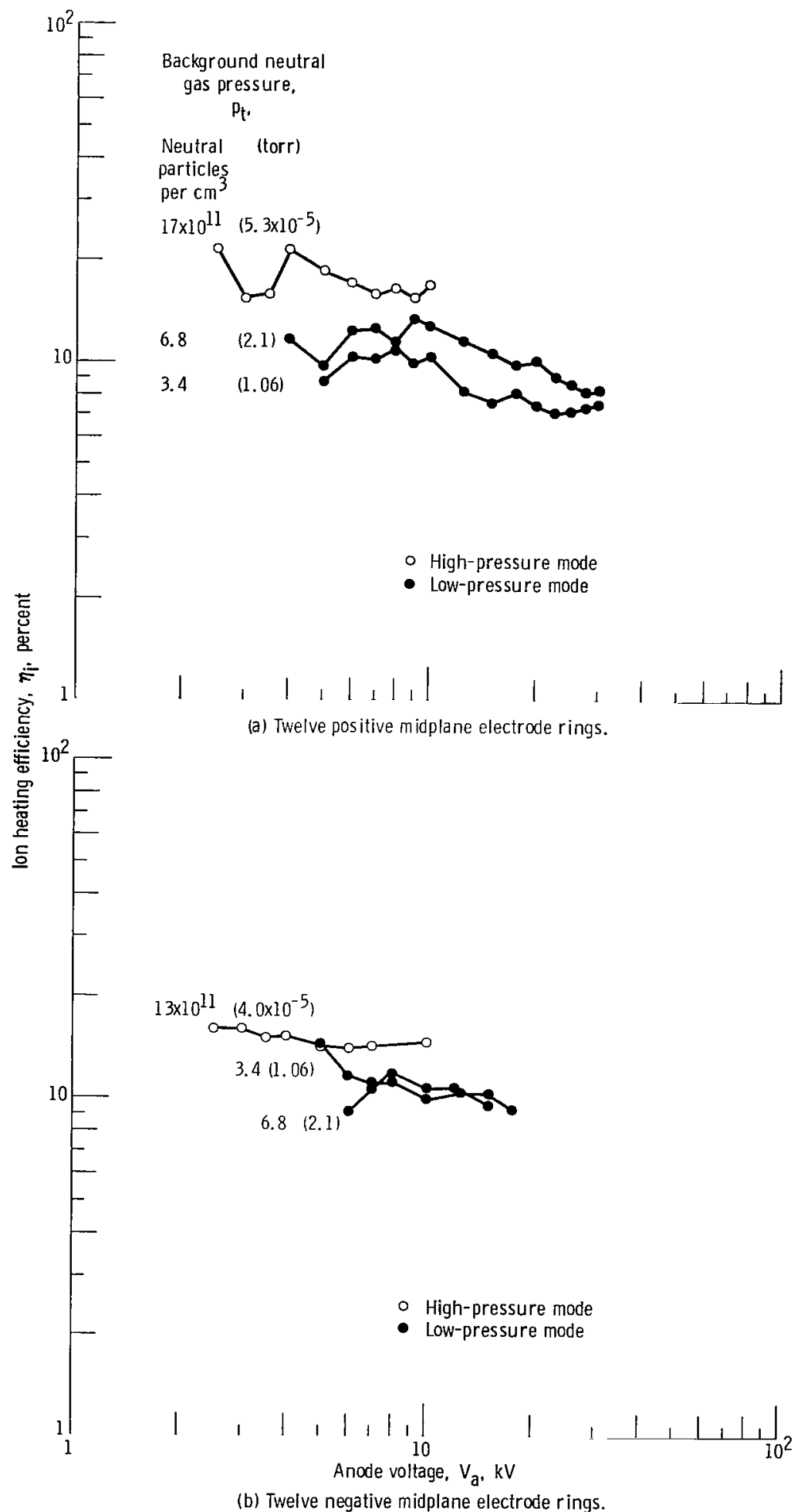

Figure 40. - Ion heating efficiency as function of anode voltage for the same conditions as figure 39 . 


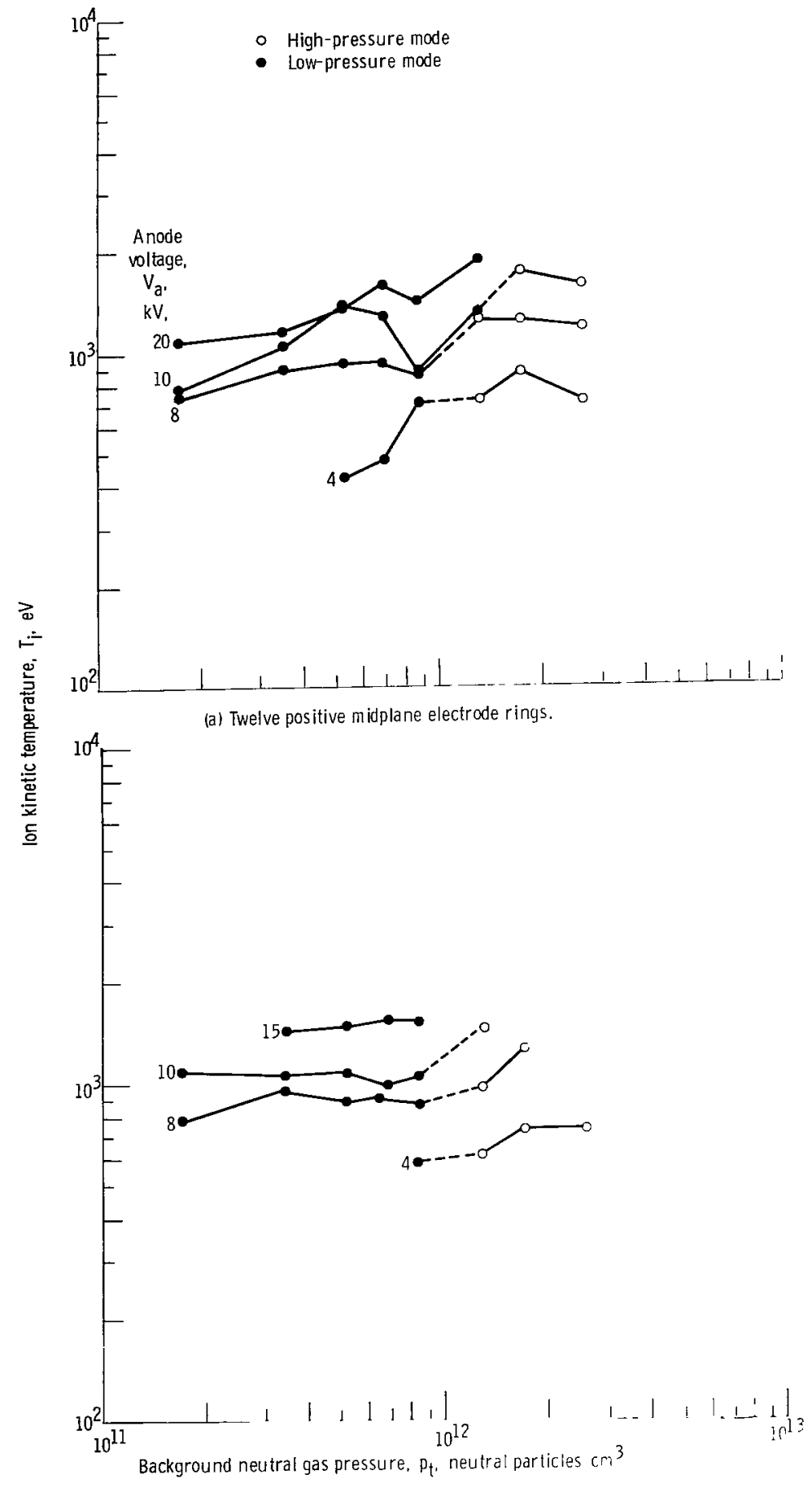

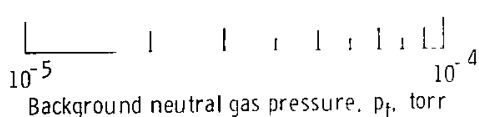

(b) Twelve negative midplane electrode rings.

Figure 4l - Ion kinetic temperature as function of background neutral gas (deuter ium) pressure for four anode voltages. Maximum magnelic field strength, 2.4 teslas. 
Figures 41(a) and (b) show ion kinetic temperature as a function of neutral background pressure for four selected values of applied anode voltage and for 12 positive and 12 negative midplane electrode rings, respectively. With the anode rings positive (fig. 41(a)) there is a slight trend of increasing ion kinetic temperature as the background pressure increases and a more marked increase in ion kinetic temperature as the discharge makes the transition from the low-pressure to the high-pressure mode of operation. With the anode rings negative (fig. 41(b)) the ion kinetic temperature is virtually independent of background neutral gas pressure, but increases as the plasma makes a transition from the low-pressure to the high-pressure mode of operation. Ion heating efficiency is shown as a function of deuterium background pressure in figures 42(a) and (b) for 12 positive and 12 negative midplane electrode rings, respectively. Efficiency increased slightly as the background pressure increased for positive electrode rings but was virtually independent of background pressure for negative electrode rings. In both configurations the efficiency improved when the plasma made a transition from the low-pressure to the high-pressure mode of operation.

Figures $43(\mathrm{a})$ and (b) show the ion kinetic temperature as a function of maximum magnetic field over a factor of 10 in magnetic field strength for 12 positive and 12 negative midplane electrode rings, respectively. The anode voltage and background pressure were held constant for the two sets of experimental runs illustrated, and these runs are characteristic of a much larger body of data. Other factors being equal, the ion kinetic temperature increased as the maximum magnetic field increased with both positive and negative midplane electrode rings. The increase in ion kinetic temperature was between a factor of 1.5 and 2 as the magnetic field was increased by a factor of 10 . The increasing trend of ion kinetic temperature with stronger magnetic fields is encouraging in that it suggests that this ion heating mechanism may possibly be scaled to magnetic fields of fusion reactor interest without suffering a penalty in ion kinetic temperature. Figures 44(a) and (b) show the ion heating efficiency, for the same sets of operating conditions shown in figure 43 , as a function of maximum magnetic field strength for 12 positive and 12 negative midplane electrode rings, respectively. In both cases, the ion heating efficiencies show an approximate increase of 1.5 to 2 as the magnetic field was increased to its maximum value. The trend line of ion heating efficiency with maximum magnetic field suggests that it could be further improved by scaling to stronger magnetic fields of fusion reactor interest.

The data presented in figures 43 and 44 show a trend with magnetic field opposite to that reported in reference 29 for the SUMMA experiment, for which the ion kinetic temperature is inversely proportional to magnetic field. 

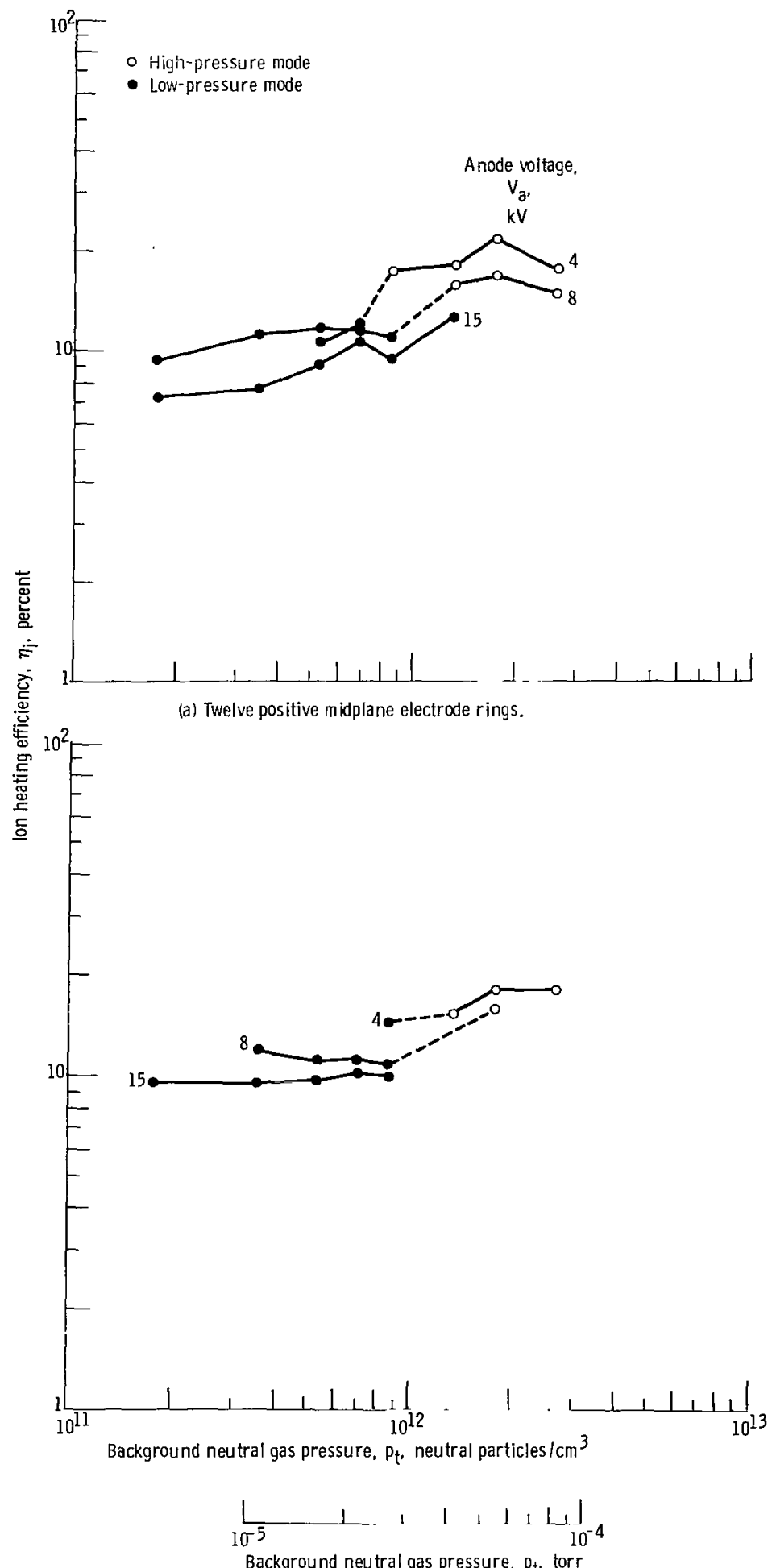

(b) Twelve negative midplane electrode rings.

Figure 42. - Ion heating efficiency as function of background neutral gas (deuterium) pressure for three anode voltages. Maximum magnetic field strength, 2.4 teslas. 


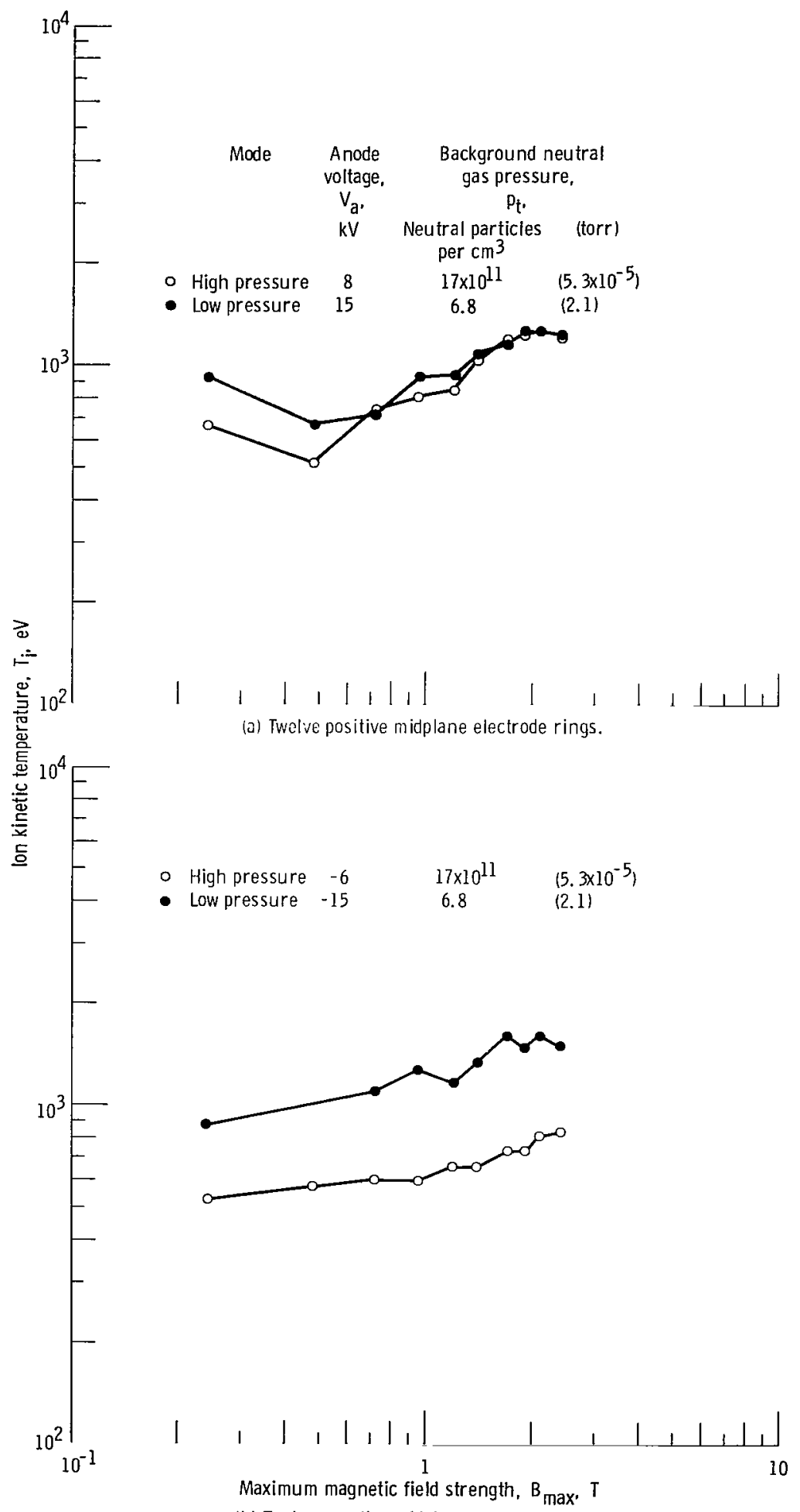

(b) Twelve negative midplane electrode rings.

Figure 43. - Ion kinetic temperature as function of maximum magnetic field strength for two background pressures of neutral deuterium gas. 


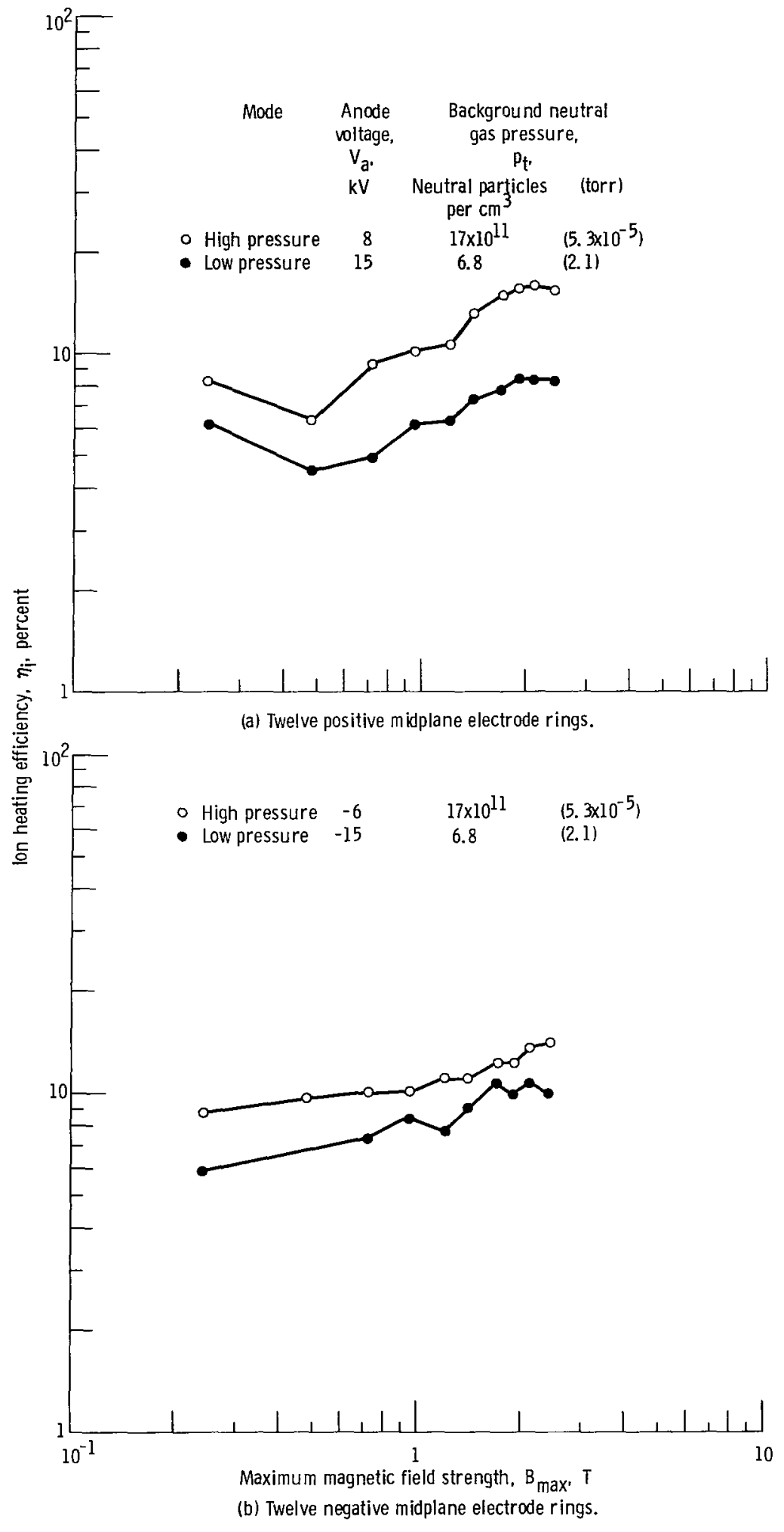

Figure 44. - Ion heating efficiency as function of maximum magnetic field strength for the same conditions as figure 43. 


\section{CONCLUSIONS}

The characteristics of the bumpy-torus plasma differed significantly, depending on whether the plasma was generated with positive or negative midplane electrode rings. Under equivalent conditions, higher plasma currents were drawn when the midplane electrodes were negative. The entire plasma ring was biased to potentials approaching those of the midplane electrodes with both electrode polarities. The radial electric field between the plasma and the grounded magnet dewars reversed, pointing radially inward toward the plasma when the plasma and the midplane electrodes were biased negatively and pointing radially outward toward the dewars with positive midplane electrode potentials.

Rotating spokes were observed at frequencies ranging from several tens of kilohertz to 1 megahertz. The structure of these spokes was investigated with an array of capacitive probes, and it was found that the rotating ion spokes extended around the entire major circumference of the torus and were in phase. The ion spokes rotated in the $\overrightarrow{\mathrm{E}} \times \overrightarrow{\mathrm{B}}$ direction in the minor azimuth; the direction of rotation reversed sign, consistent with the radial electric field resulting from a positive or negative potential on the midplane electrodes. Under high-energy-density operating conditions, the relation between the ion spoke rotational frequency and the ion kinetic temperature was consistent with that previously observed for the modified-Penning-discharge, mirror machine plasma. However, at anode voltages below 10 kilovolts and in the low-pressure mode of operation, the ion spoke rotational frequency did not obey the predicted dependence on ion kinetic temperature. When the midplane electrodes were biased to negative potentials, there was relatively poor agreement between the measured ion kinetic temperatures and the predicted ion spoke rotational frequency.

The ion spokes have minor azimuthal mode numbers as high as \pm 3 , for which three spokes exist simultaneously and rotate about the minor axis of the plasma. The peakto-peak amplitudes of the electrostatic potential fluctuations associated with these ion spokes have been observed to be as high as several kilovolts, to reach a maximum at about two-thirds of the way along the minor plasma radius, and to be typically from 10 to 20 percent of the potential applied to the midplane electrodes. The parametric behavior of the minor azimuthal mode numbers was relatively complex. In the low pressure mode of operation they tended to increase with increasing anode voltage, while in the high-pressure mode of operation they tended to decrease with increasing anode voltage.

Ions could be heated to the same kinetic temperatures with the same efficiency by the same applied potentials, regardless of whether the 12 midplane electrodes were biased to positive or negative potentials. It also made relatively little difference whether the ions were heated by one of 12 positive midplane electrode rings, although it was not possible to generate a plasma with only a single negative midplane electrode ring. When 
a plasma was generated with a single anode ring, ions were heated around the entire torus, not just in the sector in which the anode ring was located.

The ion kinetic temperatures tended to increase with anode voltage if other factors remained constant, but the ion heating efficiency tended to decrease slightly as the anode voltage was increased with both positive and negative potentials applied to the midplane electrodes. The ion kinetic temperatures and heating efficiencies were virtually independent of background neutral gas pressure, although a significant increase in both occurred when the plasma underwent a transition from the low-pressure to the highpressure mode of operation. Both the ion kinetic temperature and the ion heating efficiency increased with magnetic field over a factor of 10 in magnetic field strength for both positive and negative midplane electrode potentials.

Observation of moving peaks in the radiofrequency emission spectrum of this plasma has opened up a potentially valuable diagnostic method for determining plasma number densities in high-energy-density plasmas of fusion interest. Unfortunately, in the present series of investigations we have had no independent method of determining the absolute number density of the plasma. Thus, it is not possible to demonstrate conclusively that we have in fact observed plasma conditions at the lower hybrid frequency and that these emissions give an accurate measure of the plasma number density. However, the number densities measured by this technique appear to be proportional to the relative plasma number densities obtained from spectroscopic measurements during a previous series of investigations.

Under no conditions of operation in the current series of investigations were we able to unambiguously identify a peak in the frequency spectrum with the ion cyclotron frequency. The moving peaks that were observed were normally only 2 or 3 decibels above background for the positive midplane electrodes but were as much as 10 or 20 decibels above the background for the negative midplane electrodes. In order for the moving peaks in the radiofrequency spectrum to be used as a diagnostic of density or other plasma properties, the plasma must exist for a long enough time to permit a radiofrequency spectrum to be swept out over the relevant frequency range of interest. Since existing spectrum analyzers require times of the order of seconds to tens of seconds to make such a spectral measurement, this diagnostic method is limited to plasmas the duration of which is virtually steady-state, as is true of the bumpy-torus plasma. The current series of investigations show that this radiofrequency emission diagnostic has promise, but it remains to unambiguously demonstrate that the moving peaks in the radiofrequency spectrum are a measure of the absolute plasma number density.

Lewis Research Center,

National Aeronautics and Space Administration, Cleveland, Ohio, January 5, 1976, 506-25. 


\section{APPENDIX - SYMBOLS}

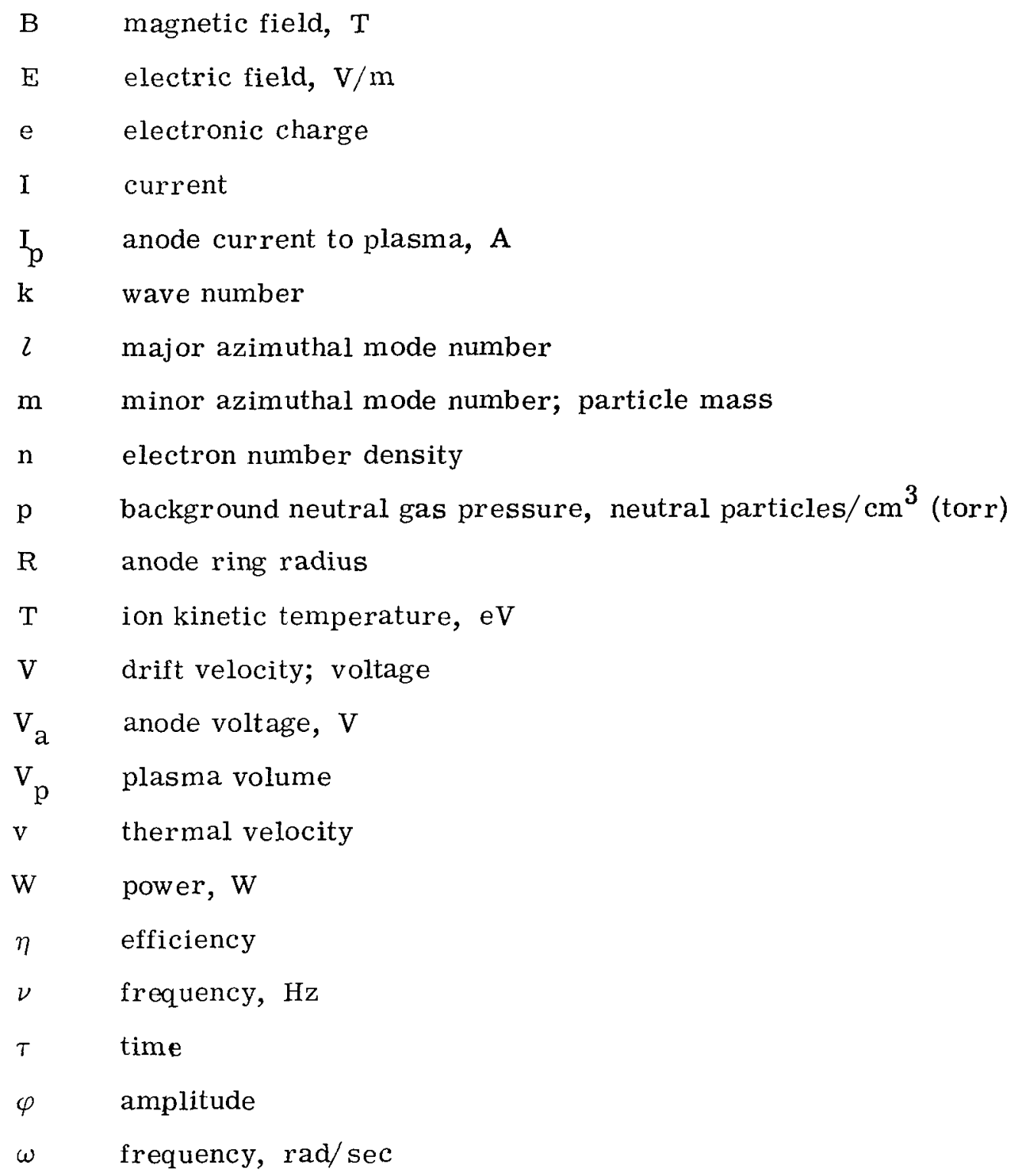
A midplane electrode (anode)
c particle confinement
ce electron cyclotron
ci ion cyclotron
D drift velocity
ES anode sheath drift 


$\begin{array}{ll}\text { e } & \text { energy confinement } \\ \text { IS } & \text { bulk plasma drift } \\ \text { i } & \text { ions } \\ \text { ith } & \text { ion thermal } \\ \text { LH } & \text { lower hybrid } \\ \text { max } & \text { maximum } \\ \text { pi } & \text { ion plasma } \\ \text { s } & \text { spoke } \\ \text { t } & \text { tank } \\ \text { o } & \text { initial value }\end{array}$




\section{REFERENCES}

1. Roth, J. Reece; Gerdin, Glenn A. ; and Richardson, Richard W.: Characteristics of NASA Lewis Bumpy-Torus Plasma Generated with Positive Applied Potentials. NASA TN D-8114, 1976.

2. Roth, J. Reece: Plasma Stability and the Bohr - Van Leeuwen Theorem. NASA TN D-3880, 1967.

3. Roth, J. Reece, et al. : Characteristics and Performance of a Superconducting "Bumpy Torus" Magnet Facility for Plasma Research. NASA TN D-7353, 1973.

4. Roth, J. Reece, et al.: A 12-Coil Superconducting "Bumpy Torus" Magnet Facility for Plasma Research. Fifth Applied Superconductivity Conference, Inst. of Electrical and Electronics Engrs., 1972, pp. 361-366.

5. Roth, J. R., et al. : Performance of a 12-Coil Superconducting "Bumpy Torus" Magnet Facility. Proceedings of Technology of Controlled Thermonuclear Fusion Experiments and the Engineering Aspects of Fusion Reactors. Am. Nuclear Soc., 1974, pp. 409-425.

6. Roth, J. Reece: Origin of Hot Ions Observed in a Modified Penning Discharge. Phys. Fluids, vol. 16, no. 2, Feb. 1973, pp. 231-236.

7. Roth, J. Reece: Hot Ion Production in a Modified Penning Discharge. IEEE Trans. Plasma Sci., vol. PS-1, no. 1, Mar. 1973, pp. 34-45.

8. Roth, J. Reece; Richardson, Richard W. ; and Gerdin, Glenn A. : Initial Results from the NASA Lewis Bumpy Torus Experiment. NASA TM X-71468, 1973.

9. Richardson, Richard W.: Spectroscopic Results in Helium from the NASA Lewis Bumpy Torus Plasma. NASA TM X-71569, 1974.

10. Richardson, Richard W.: Effect of Anode Ring Arrangement on the Spectroscopic Characteristics of the NASA Lewis Bumpy Torus Plasma. NASA TM X-71636, 1974.

11. Richardson, Richard W.: Determination of Electron Temperature in a Penning Discharge by the Helium Line Ratio Method. NASA TM X-71677, 1975.

12. Gerdin, Glenn A.: Radio Frequency Studies in the NASA Lewis Bumpy Torus. NASA TM X-71567, 1974.

13. Gerdin, Glenn A.: Spoke Wavenumbers and Mode Transitions in the NASA Lewis Bumpy Torus. NASA TM X-71624, 1974.

14. Roth, J. Reece: Ion Heating and Containment in the NASA Lewis Bumpy Torus Plasma. NASA TM X-71630, 1974. 
15. Kambic, George X.: Heavy Ion Beam Probe Measurements of Radial Potential Profiles in the Modified Penning Discharge. NASA TM X-71643, 1974.

16. Persson, Hans: Time-Dependent Outgassing and Impurities in the NASA Lewis Bumpy Torus. NASA TM X-71639, 1974.

17. Stix, Thomas H.: The Theory of Plasma Waves. McGraw-Hill Book Co., Inc., 1962 , p. 32 .

18. Burtis, W. J.: Electron Concentrations Calculated from the Lower Hybrid Resonance Noise Band Observed by OGO-3. J. Geophys. Res., vol. 78, no. 25, Sept. 1973, pp. 5515-5523.

19. Hirose, A.; and Alexeff, I.: Electrostatic Instabilities Driven by Currents Perpendicular to an External Magnetic Field. Nucl. Fusion, vol. 12, no. 3, May 1972, pp. 315-323.

20. Stix, Thomas H.: Radiation and Absorption via Mode Conversion in an Inhomogeneous Collision-Free Plasma. Phys. Rev. Lett., vol. 15, no. 23, Dec. 1965, pp. $878-882$.

21. Moresco, M.: Observation of Extraordinary Wave Propagation Near the Lower Hybrid Resonance Frequency. Phys. Letts., vol. 45A, no. 1, Aug. 1973, pp. 65-67.

22. Hooke, W. M.; and Bernabei, S.: Direct Observation of Waves Propagating Near the Lower-Hybrid-Resonance Frequency, Phys. Rev. Lett., vol. 28, no. 7, Feb. 1972, pp. 407-410.

23. Ashby, D. E. T. F.; and Paton, A.: A High Frequency Electrostatic Instability in a Magnetically Guided Plasma Stream. Plasma Phys., vol. 9, no. 4, Apr. 1967, pp. 359-368.

24. Schmidt, J. A.: High Impedance Langmuir Probes. Rev. Sci. Instrum., vol. 39, no. 9 , Sept. 1968 , pp. 1297-1299.

25. Roth, J. Reece; Krawczonek, Walter M. : Paired Comparison Tests of the Relative Signal Detected by Capacitive and Floating Langmuir Probes in Turbulent Plasma from 0.2 to $10 \mathrm{MHz}$. Rev. Sci. Instrum., vol. 42, no. 5, May 1971, pp. 589-594.

26. Roth, J. Reece: Experimental Study of Spectral Index, Mode Coupling, and Energy Cascading in a Turbulent, Hot-Ion Plasma. Phys. Fluids, vol. 14, no. 10, Oct. 1971, pp. 2193-2202.

27. Roth, J. R.: Energy Distribution Functions of Kilovolt Ions in a Modified Penning Discharge. Plasma Phys., vol. 15, no. 10, Oct. 1973, pp. 995-1005. 
28. Valckx, F. P. G.: Electrostatic Analyzer for the Detection of Fast Neutral Particles. NASA TT F-11, 458, 1968.

29. Reinmann, John J.; et al.: Hot Ion Plasma Heating Experiments in SUMMA. NASA TM X-71559, 1974, fig. 15. 


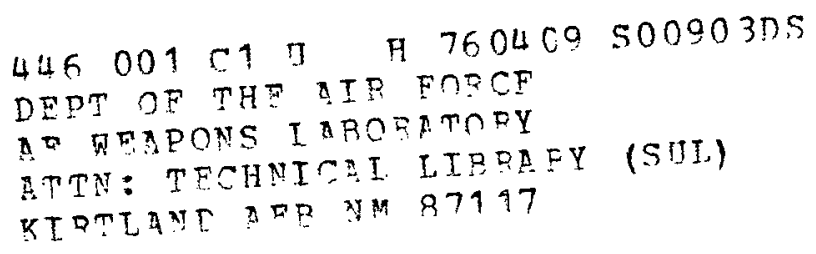

"The aeronautical and space activities of the United States shall be conducted so as to contribute... to the expansion of buman knowledge of phenomena in the atmosphere and space. The Administration shall provide for the widest practicable and appropriate dissemination of information concerning its activities and the results thereof."

-National Aeronautics and SPACE ACt of 1958

\section{NASA SCIENTIFIC AND TECHNICAL PUBLICATIONS}

TECHNICAL REPORTS: Scientific and technical information considered important, complete, and a lasting contribution to existing knowledge.

TECHNICAL NOTES: Information less broad in scope but nevertheless of importance as a contribution to existing knowledge.

TECHNICAL MEMORANDUMS:

Information receiving limited distribution because of preliminary data, security classification, or other reasons. Also includes conference proceedings with either limited or unlimited distribution.

CONTRACTOR REPORTS: Scientific and technical information generated under a NASA contract or grant and considered an important contribution to existing knowledge.
TECHNICAL TRANSLATIONS: Information published in a foreign language considered to merit NASA distribution in English.

SPECIAL PUBLICATIONS: Information derived from or of value to NASA activities. Publications include final reports of major projects, monographs, data compilations, handbooks, sourcebooks, and special bibliographies.

TECHNOLOGY UTILIZATION PUBLICATIONS: Information on technology used by NASA that may be of particular interest in commercial and other non-aerospace applications. Publications include Tech Briefs, Technology Utilization Reports and Technology Surveys.

Defails on the availability of these publications may be obtained from:

SCIENTIFIC AND TECHNICAL INFORMATION OFFICE 